


\section{SPINTRONIC EFFECTS AT NICKEL-GRAPHENE INTERFACES}

ELMER VAN GEIJN 



\section{SPINTRONIC EFFECTS AT \\ NICKEL-GRAPHENE INTERFACES}

\section{DISSERTATION}

to obtain

the degree of doctor at the University of Twente, on the authority of the rector magnificus, prof. dr. T.T.M. Palstra, on account of the decision of the graduation committee, to be publicly defended on Friday 1o February 2017 at 14.45

by

ELMER VAN GEIJN

born on 25 October 1981

in Heerlen, the Netherlands 
This dissertation has been approved by:

prof. dr. ir. W.G. van der Wiel

dr. ir. M.P. de Jong

Members of the graduation committee:

prof. dr. ir. W.G. van der Wiel University of Twente (promoter)

dr. ir. M.P. de Jong University of Twente (co-promoter)

prof. dr. P.M.G. Apers University of Twente (chairman, secretary)

prof. dr. ir. L. Abelmann University of Twente

prof. dr. ir. A. Brinkman University of Twente

$\begin{array}{lll}\text { prof. dr. } & \text { P.J. Kelly University of Twente }\end{array}$

prof. dr. ing. A.J.H.M. Rijnders University of Twente

prof. dr. P. Seneor Unité Mixte de Physique CNRS/Thales

prof. dr. ir. H.J.M. Swagten Eindhoven University of Technology

Copyright $@ 2017$ by Elmer van Geijn

Cover: Standing shards of (multilayer) graphene, electron microscope image by Johnny Sanderink

Printed by: Gildeprint - Enschede

ISBN: $\quad$ 978-90-365-4288-3

DOI: $\quad 10.3990 / 1.9789036542883$ 
"Nobody ever figures out what life is all about, and it doesn't matter. Explore the world. Nearly everything is really interesting if you go into it deeply enough."

- Richard P. Feynman

To Irma,

I love you! 

As I am writing this there are just a few hours left before this document has to be sent off for printing. I tend to think of myself as someone who is quite resilient to stress, but the last few weeks have felt as one big exception to that rule. And there will be a big sense of relief when the feeling of guilt, or maybe rather discomfort, when spending time on something other than improving this little book is gone. It is also at this moment, with the rest of this thesis pretty much finished, that I can look back at these last four and a half years with some clarity of thought.

Doing a PhD has been a wonderful experience. Of course there have been times of frustration and demotivation when stuff didn't work, again, but being allowed to do scientific research for the sake of scientific research in an inspiring environment has felt as a big privilege. A privilege for which I have to thank Michel de Jong, my daily supervisor, who was willing to hire me to fill a position within one of his projects. It has been a pleasure to work together and I highly appreciate all the provided feedback and guidance. The combination of a friendly character and a vast theoretical and experimental knowledge and understanding is inspiring.

Next I would like to thank my promoter, Wilfred van der Wiel, for giving me the opportunity to do my $\mathrm{PhD}$ in his research group. The great atmosphere and strong social cohesion he promotes within the NanoElectronics family have been important factors in making my time as a $\mathrm{PhD}$ such a pleasant one. And these things are of course just as much a merit of the rest of the permanent staff of the group: Floris, also indispensable as coordinator on the football pitch. Johnny, helping out with equipment while chatting about gardening, travelling and other topics form the "real" world, Thijs, Karen, Martin, Hajo and Peter, all making live a lot easier for us $\mathrm{PhDs}$ and contributing to the great ambiance of the group. 
The experiments I performed during my PhD wouldn't have been possible without access to the cleanroom facilities of the MESA+ Institute for Nanotechnology. I owe gratitude to all members of the team that make sure everything stays up and running. I am especially thankful to Hans Mertens who was always willing to help out by showing me how to use equipment and by discussing the possibilities of experimental and untested processes. And who also stayed friendly when I occasionally provided him with additional maintenance work when my process proved a bit too experimental for the precious equipment.

During the years there have also been several students I supervised that contributed to my research for their bachelor's or master's graduation project. Thank you for your valuable additions Jelmer, Kees and Remco.

But, all work and no play makes Jack a dull boy, and so there are the numerous people who have made sure that the last four and a half years were also full of fun. First of all Chris and Matthias with whom I shared the best office on this side of the universe. Thanks for all the good times, our awesome office warming party, drinking leftover beers, for putting up with my terrible puns and for standing beside me as my paranymphs during the public defense of my thesis. Ksenia, our office mate from another office, always kind and always in for tequila, and Kurt, starting philosophical physics discussions when I should actually be doing something useful, like writing a thesis. Filipp, thanks for the great hiking trip and for getting me to play water polo. Joost, there will always be a spot reserved for you on our office couch. Janine, thanks for the parties with "weerwolven" around the camp fire. Dilu and Sergey, my fellow members of the academy of young fathers in science. Derya, thanks for the good times making movies for Janine and for Ksenia and Kurt. Thank you and all the best to all the other current and former members of the NanoElectronics family.

I am grateful to all my friends outside of my little corner of academia for still sending me invitations to birthdays and other social events although I haven't attended many of them in the last year. And to all members of my and Irma's family who have always 
been supportive and provided positive encouragement more than once.

And then there are those who provide the unconditional moral support. Agnes and Edgar, mom and dad, thank you for providing the parental love and support that acts as the safety net allowing me to go through life with confidence. Oscar, my little man of two years old, thanks you for helping daddy relax and unwind by a powerful combination of playing with Lego bricks, going to the swimming pool and lots and lots of hugs. And last but not least Irma, thank you for always being understanding and supportive, for accepting all my flaws, and for not even once, in the last few months when my mind was preoccupied with finishing my thesis, complaining about my absent-mindedness and minimal contribution to family and household affairs.

Elmer van Geijn, January 2017 

PREFACE vii

LIST OF ACRONYMS Xiii

1 INTRODUCTION 1

1.1 A short history of (organic) spintronics ....... I 1

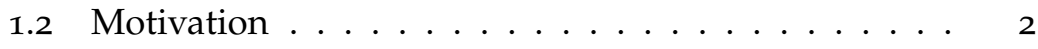

1.3 Outline ....................... 4

2 THEORETICAL BACKGROUND 5

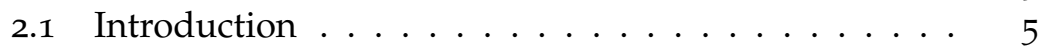

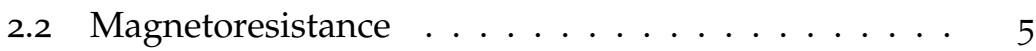

2.3 Interfaces in spintronics ........... 13

3 FABRICATION OF DEVICES WITH MULTILAYER GRAPHENE 15

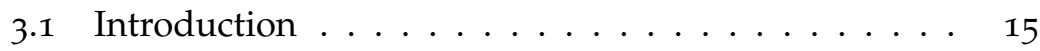

3.2 Multilayer graphene on nickel ........... 16

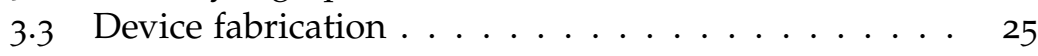

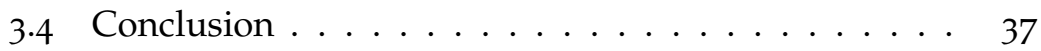

4 MAgNetoresistanCE OF DEVICES CONTAINING NICKELGRAPHENE INTERFACES 39

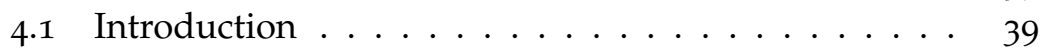

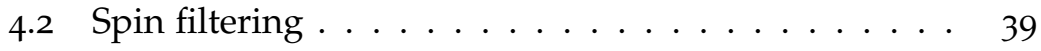

4 Tunnelling anisotropic magnetoresistance ...... 45

4.4 Conclusion ................... 50

5 SOlid STATE EPITAXIAL GROWTH OF NiCOB ON GRAPHITE 51

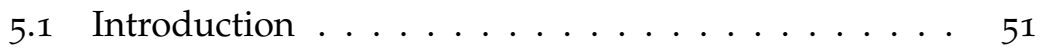

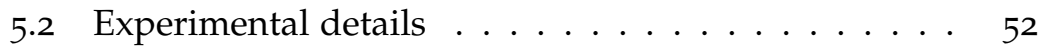

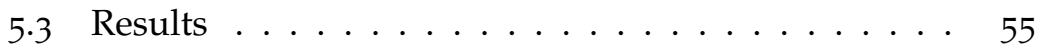

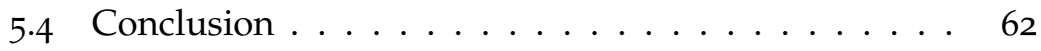


xii

CONTENTS

6 ELECTRONIC AND MAGNETIC PROPERTIES OF TTF AND TCNQ COVERED COBALT THIN FILMS 63

6.1 Introduction . . . . . . . . . . . . 63

6.2 Materials and methods ............ 65

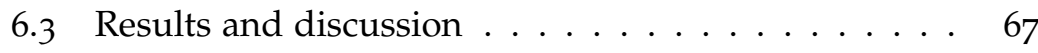

6.4 Conclusion .................... 76

7 CONCLUSION AND OUTLOOK 79

BIBLIOGRAPHY I

SAMPLE FABRICATION RECIPE XIII

SUMMARY XIX

SAMENVATTING XXIII

LIST OF PUBLICATIONS XXVII 


\section{LIST OF ACRONYMS}

\begin{tabular}{|c|c|}
\hline AFM & atomic force microscope \\
\hline AMR & anisotropic magnetoresistance \\
\hline BHF & buffered hydrofluoric acid \\
\hline CVD & chemical vapour deposition \\
\hline DOS & density of states \\
\hline EBL & electron beam lithography \\
\hline GMR & giant magnetoresistance \\
\hline HOPG & highly-oriented pyrolytic graphite \\
\hline MFM & magnetic force microscopy \\
\hline MR & magnetoresistance \\
\hline MTJ & magnetic tunnel junction \\
\hline SEM & scanning electron microscope \\
\hline $\mathrm{SOC}$ & spin-orbit coupling \\
\hline TAMR & tunnelling anisotropic magnetoresistance \\
\hline TCNQ & tetracyanoquinodimethane \\
\hline TMR & tunnelling magnetoresistance \\
\hline TTF & tetrathiafulvalene \\
\hline VSM & vibrating sample magnetometer \\
\hline XAS & X-ray absorption spectroscopy \\
\hline $\mathrm{XMCD}$ & X-ray magnetic circular dichroism \\
\hline XPS & X-ray photoelectron spectroscopy \\
\hline XRD & $\mathrm{X}$-ray diffraction \\
\hline
\end{tabular}





\section{INTRODUCTION}

\section{I A SHORT HISTORY OF (ORGANIC) SPINTRONICS}

In the middle of the 1930s Mott $(1935,1936 a, b)$ published a number of papers in which he set out to explain some anomalies in the transport properties of transition metals. For instance, experimental observations had shown that the differences in conductivities between transition and noble metals are larger than one would expect based on a comparison of the number of available charge carriers (effective free electrons). Mott showed that the lower conductivity in the transition metals could be explained by a high probability of delocalised s-electrons scattering into unoccupied $d$-states, which have much lower mobilities. He continues to say:

"Since the unoccupied $d$ states are responsible for the ferromagnetism or high paramagnetism of the transition elements, there is a direct connexion between the magnetic properties and the electrical conductivity." (Mott, 1936a)

a statement that can be seen as the birth of the field of spintronics.

The name spintronics is a combination of the terms spin and electronics. The concept of electron spin, an intrinsic angular momentum, as the additional quantum property needed for Pauli's exclusion principle to work, was introduced by Uhlenbeck and Goudsmit (1926) a few years after the experiments of Gerlach and Stern (1922) had shown that the orientation of angular momentum was quantized. The field of spintronics focusses on studying the properties and possibilities of this spin of the electron in solid-state systems in addition to its electronic charge. 
The works of Jullière (1975) and Tedrow and Meservey (1971) that show spin dependent tunnelling through a barrier to and from a ferromagnetic metal, are arguably the first reports of spintronic devices. However, it were the experimental evidence of spin injection into a normal metal presented by Johnson and Silsbee (1985) and the Nobel Prize winning discovery of giant magnetoresistance (GMR) by Albert Fert and Peter Grünberg (Baibich et al., 1988; Binasch et al., 1989) that really unlocked the potential of spintronics and have led to technological applications such as magnetic sensors, hard drive read heads and magnetoresistive memory (MRAM). Up until the present day the field of spintronics is one of active scientific research that still leads to the discovery of novel physics.

The demonstration of magnetoresistance in a device with an organic semiconductor separating the ferromagnetic leads by Dediu et al. (2002) marks the beginning of the field of organic spintronics (Dediu et al., 2009; Naber et al., 2007). Technological advantages such as low fabrication costs and the seemingly endless flexibility in material properties owing to the vast knowledge within the field of organic chemistry have been a driving factor for the research of organic electronics, with the invention of the organic LED as a notable commercial success (Tang and VanSlyke, 1987). Furthermore, the use of organic materials brings additional benefits that are particularly interesting for spintronic applications: The low atomic number of carbon and other elements typically found in organic materials and the lack of a nuclear magnetic moment in the most abundant carbon isotope, ${ }^{12} \mathrm{C}$, for instance, are both properties that increase the longevity of spin polarisation in transport, a key requirement for spintronic applications and an important incentive for the emergence of the field of organic spintronics.

\subsection{MOTIVATION}

The general motivation of this project comes from the recognition of the importance of interfaces for the generation and injection of spin polarisation (Sanvito, 2010). We set out to contribute to the knowledge of the physics of hybrid interfaces whilst also showing 
the possibilities of fabricating devices with a high level of control over the interfacial structure.

Since the successful isolation of graphene (Novoselov et al., 2004), the peculiar properties (Castro Neto et al., 2009) of this two-dimensional allotrope of carbon have attracted much scientific interest. The perfect spin filtering at the interface between nickel and graphene predicted by Karpan et al. $(2007,2008)$ and the experimentally observed long spin relaxation lengths in graphene by Tombros et al. (2007) have led us to the choice of the nickel-graphene interface as the foundation for our devices. Several possible pathways to fabricate devices containing nickel-graphene interfaces have been explored and in this thesis we report on the most successful and promising ones. One of the developed methods has led to the fabrication of complete devices on which transport measurements have been performed.

Besides our main project we were fortunate enough to have appointed to us some weeks of access to the synchrotron radiation source at the MAX-lab facility in Lund, Sweden. The time spent there was used to investigate the interfacial properties of the hybrid interface of a thin cobalt film with organic molecular layers of tetrathiafulvalene (TTF) and tetracyanoquinodimethane (TCNQ). 


\subsection{OUTLINE}

Below we present a short overview of the contents of the remaining chapters of this thesis.

CHAPTER 2 - To provide the reader with some theoretical background we introduce some of the physical concepts that are of importance in this research. The chapter contains an introduction to magnetoresistance and some of the different types thereof, and some remarks on hybrid interfaces.

CHAPTER 3 - Discusses the growth and characterisation of graphene on nickel and provides a detailed description of the developed fabrication process for devices for studying transport across the nickel-graphene interface.

CHAPTER 4 - Transport measurement were performed on the devices containing nickel-graphene interfaces fabricated using the methods described in Chapter 3. This chapter presents and discusses the results of those measurements.

CHAPTER 5 - In this chapter we investigate an alternative fabrication method for creating epitaxial ferromagnetic layers on top of graphene by carefully crystallising an initially amorphous ferromagnetic alloy.

CHAPTER 6 - Gives an overview of the experiments that were performed at the MAX-lab synchrotron radiation source. During out time there we investigated the electronic and magnetic properties of thin cobalt films covered with TTF and TCNQ molecular layers.

CHAPTER 7 - Contains some concluding remarks and a short outlook based on the results of this thesis.

SAMPLE FABRICATION RECIPE - In the back matter of the book a specification is given of the fabrication process described in Chapter 3 and used for the devices of Chapter 4 . 


\section{THEORETICAL BACKGROUND}

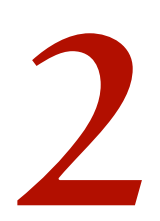

\subsection{INTRODUCTION}

In this chapter we will provide some theoretical background of the physical phenomena that we discuss in this thesis. In very general terms the subject of this project is the study of spintronic effects at interfaces. By giving a brief introduction to the topic of magnetoresistance and discussing some magnetic and electronic properties of interfaces, we hope to provide the reader with some perspective on this interesting research field. For a more comprehensive treatment of the various subjects the reader is referred to literature.

\subsection{MAGNETORESISTANCE}

Magnetoresistance (MR) is the catch-all name for a change in resistance of a material or device under the influence of an externally applied magnetic field. The term can be misleading since it is not an actual physical quantity like electrical resistance, nor does it imply a specific causal mechanism, it merely refers to the observed phenomenon in general. As a quantitative measure for the observed effect the relative change in the resistance $R$, the so called MR ratio, is often presented as a percentage:

$$
\operatorname{MR} \operatorname{ratio}(x)=\frac{R(x)-R\left(x_{0}\right)}{R\left(x_{0}\right)} \cdot 100 \%
$$

Here $x$ indicates the magnetic quantity that is varied, such as magnetic field strength or direction, with $x_{0}$ a suitably chosen reference value. It is obvious from this definition that the value for the MR 
ratio can be strongly influenced by the choice of reference configuration, and so great care must be taken when comparing values between experiments.

All metals actually show so called ordinary MR: an increase in resistance under an applied magnetic field (Lüthi, 1959). The effect is strongest for a field perpendicular to the current path and mainly caused by Lorentz forces pushing the electrons to the surface of the conductor where they experience increased scattering. Obviously, this effect is also influenced by the sample geometry.

In ferromagnetic metals there is a second source of MR. As already mentioned in the introduction, the resistivity of the transition metals can be explained by the scattering of current carrying $s$-electrons into $d$-states with much lower mobilities (Mott, 1935). Additionally, the spin down and spin up electrons can be treated as belonging to two separate parallel currents (Campbell et al., 1967; Fert and Campbell, 1968; Mott, 1936a). If we assume that the spin scattering probability is much lower than the probability for momentum scattering these two currents can be treated as being non-interacting. In a ferromagnetic metal below its Curie temperature the exchange shifted $d$-states belonging to the majority spin direction have a higher occupancy than those for the minority spin, leading to different scattering probabilities and thus different resistances for the s-electrons in each spin system. The resulting total resistance change depends on the degree of magnetic saturation in the material.

In the following overview, that is in no way meant to be exhaustive, we will describe some other forms of MR and their underlying principles.

\section{Anisotropic magnetoresistance}

Anisotropic magnetoresistance (AMR) is the first form of MR to have been discovered and reported. Thomson (1856) describes an increase of the resistance of pieces of nickel and iron when an external magnetic field is applied in the direction of current flow and a 
decrease of resistance when the field is perpendicular to the current. AMR only occurs in ferromagnetic metals and depends on the direction of magnetisation of the material relative to the current flow:

$$
R=R_{\perp}+R_{\text {AMR }} \cos ^{2}(\theta),
$$

where $R_{\perp}$ is the resistance with the external magnetic field perpendicular to the current direction, $R_{\mathrm{AMR}}$ the difference in resistance between parallel and perpendicular orientation of the magnetic field with respect to the current flow, and $\theta$ the angle between the directions of current flow and magnetisation.

It is difficult to explain the cause of AMR in an intuitive way and for an in-depth discussion of the various theoretical models the reader is referred to literature. It arises from higher order terms in the corrections for spin-orbit coupling (SOC), the interaction between a particle's spin and its motion, to the Hamiltonian. In an applied magnetic field these corrections lead to anisotropic mixing of spin up and spin down states, and shifts in $d$-states around the Fermi energy, influencing the scattering probabilities for different states asymmetrically (Berger, 1964; Malozemoff, 1986).

Typical values for the AMR ratio range up to $3 \%$ for nickel, cobalt and iron and up to $25 \%$ for ferromagnetic alloys (McGuire and Potter, 1975).

\section{Giant magnetoresistance}

By combining ferromagnetic materials with normal metals MR effects can be exploited to create structures that exhibit much higher MR ratios. When a current passes through a ferromagnetic metal the asymmetry in resistance for majority and minority spin electrons leads to an imbalance between the number of electrons of each spin orientation in the current. The current is now said to be spin polarised. Moreover, because of this transport asymmetry, the resistance experienced by an already spin polarised current when passing into a ferromagnetic metal depends on the relative orientation of the spin polarisation of the current with respect to the 
magnetisation of the metal. Besides the aforementioned scattering between $s$-and $d$-states, spin dependent scattering effects at the interface with the ferromagnet also contribute to this effect (Hall et al., 1997).

A highly simplified model can be formulated to illustrate the effect: We send an unpolarised current through a ferromagnetic layer with a fixed orientation of the magnetisation. We define the spin up direction to be identical to the direction of magnetisation of this layer. The resistances experienced by a spin up, $\mathrm{R}_{\uparrow}$, or spin down, $R_{\downarrow}$, electron are defined as

$$
R_{\uparrow, \downarrow}=R_{0} \mp \delta R
$$

with $R_{0}$ the average of the resistances for both spin orientations, and $\delta R$ half the resistance difference. The right-hand side has a minus (plus) sign when describing the resistance for spin up (down) electrons.

The resulting spin polarised current is transported to a second identical ferromagnetic layer. In practice, this is accomplished by putting a very thin (a few nm) layer of a normal metal between the two ferromagnets. This decouples their magnetisations and allows a current to flow between the two. Naturally, this will lead to a finite loss of spin polarisation, which we will neglect in our naive model. This second ferromagnetic layer has its magnetisation either identical to the first layer, parallel, or rotated by exactly $180^{\circ}$, antiparallel. In the first case an electron with a certain spin orientation experiences the same resistance in both ferromagnetic layers, in the latter configuration the plus and minus signs in the right-hand side of equation 2.3 are interchanged for the second ferromagnetic layer. 
Comparing the total resistance in the parallel, $R_{\mathrm{P}}$, and anti-parallel, $R_{A P}$, situations we find

$$
\left.\begin{array}{rl}
R_{P} & =\left(\frac{1}{2\left(R_{0}+\delta R\right)}+\frac{1}{2\left(R_{0}-\delta R\right)}\right)^{-1} \\
& =R_{0}-\frac{(\delta R)^{2}}{R_{0}} \\
R_{A P} & =\left(2 \cdot \frac{1}{\left(R_{0}+\delta R\right)+\left(R_{0}-\delta R\right)}\right)^{-1} \\
& =R_{0}
\end{array}\right\} R_{P}<R_{A P} .
$$

Leading to a lower resistance when both ferromagnetic layers have the same magnetisation direction. For GMR to occur it is imperative that the spin polarisation emerging from the first ferromagnet is, at least partly, retained when reaching the second ferromagnet.

The first experimental observations of this effect by Baibich et al. (1988) and Binasch et al. (1989) were rewarded with the 2007 Nobel Prize in Physics, and implicitly proved the possibility to transport spin information. The first reports measured GMR ratios approaching $50 \%$ and values above $100 \%$ at room temperature have been reached. These large ratios were measured in devices with stacks of many alternating non-magnetic and ferromagnetic layers where the effect is significantly enhanced compared to our model with just two ferromagnetic and one non-magnetic layer.

\section{Tunnelling magnetoresistance}

Tunnelling magnetoresistance (TMR) was actually discovered more than ten years before GMR by Jullière (1975) when he measured an MR effect in junctions of an insulating germanium layer sandwiched between layers of cobalt and nickel. And although the devices exhibiting these two types of MR seem similar, the underlying principles are quite different. Tedrow and Meservey (1971, 1973) showed that the tunnelling current from a ferromagnetic film is spin polarised. The device they used is a stack consisting of a superconducting aluminium film and a ferromagnetic metal separated by an insulating tunnel barrier. The superconductive band gap can be 


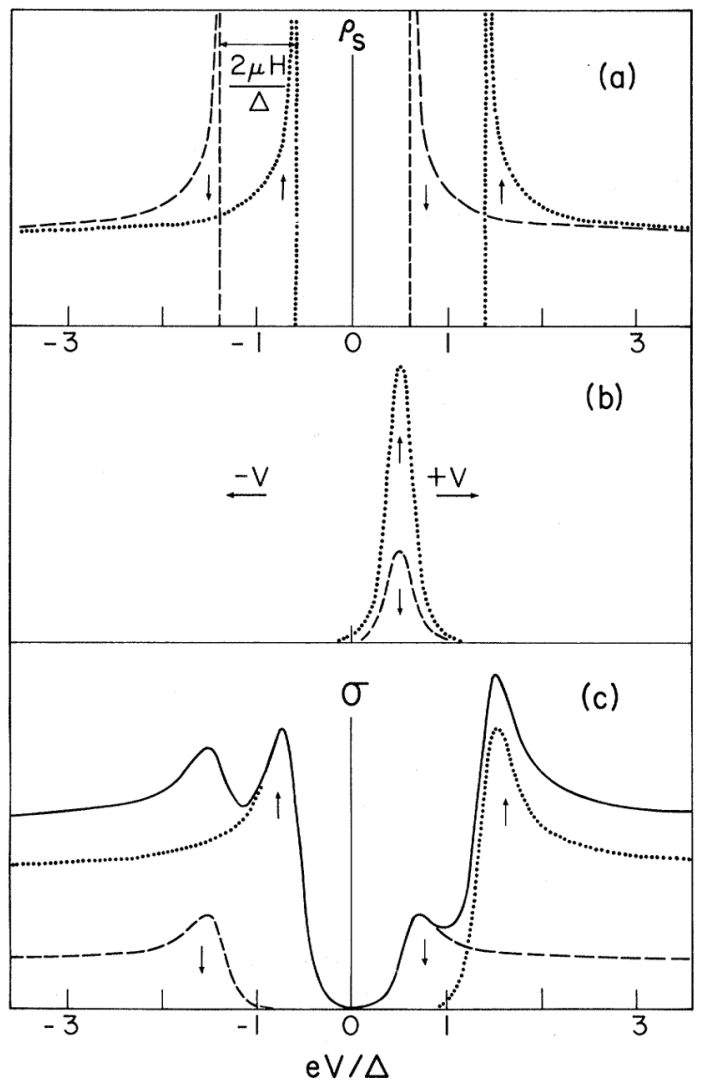

Figure 2.1: Theoretical tunnelling between superconductor and ferromagnetic metal. (a) Shows the superconductive density of states with spin splitting due to an external magnetic field. (b) The thermally broadened spin up and down states available for tunnelling around the Fermi energy in the ferromagnet. (c) Convolution of (a) and (b) yielding the conductance for individual spin orientations (dotted and dashed) and the total conductance (solid line). The spin polarisation of the tunnel current can be calculated from the relative heights of the peaks in the total conductance. (Reprinted figure with permission. From Tedrow and Meservey (1973) Copyright (C) 1973 by the American Physical Society.) 
spin split by an external magnetic field and be "swept" passed the Fermi level of the ferromagnet by applying a bias voltage over the stack. Since the tunnelling rate is proportional to the density of states (DOS) at both sides of the tunnel barrier, the degree of spin polarisation of states at the Fermi energy in the ferromagnet can be deduced from the acquired conductance plot (figure 2.1).

In a TMR device, or magnetic tunnel junction (MTJ), the superconducting film is replaced by a second ferromagnetic film. Both ferromagnetic films have a spin polarised DOS and the spin polarisation is conserved during tunnelling, so we can apply a two-current model analogous to the case of GMR. With two identical ferromagnetic layers with parallel alignment of their magnetisations, one spin state has a high DOS on both sides of the barrier, and thus a high tunnelling rate, the other spin state has a low DOS and a low tunnelling rate. For anti-parallel alignment both spin states have the same tunnelling rate with a high DOS on one side of the barrier and a low DOS on the other. A similar calculation to that for GMR in equation 2.3 can be made to show that the parallel configuration has a lower resistance than the anti-parallel one. Slonczewski (1989) shows that this picture is too simple and that exchange interaction effects at the interfaces of the ferromagnetic layers and between the ferromagnetic layers influence the tunnelling transport of spin polarised carriers.

MTJs started gaining attention when Moodera et al. (1995) reported reproducible large TMR ratios, $>10 \%$, at room temperature. $\mathrm{Ab}$ initio calculation for MTJs with thin magnesium oxide tunnel barriers (Butler et al., 2001; Mathon and Umerski, 2001) showed that barrier properties have to be taken into account. Especially in crystalline systems tunnelling probabilities strongly depend on the symmetry of the involved states and the properties of interface states. These results have been substantiated by reports of ever increasing TMR ratios in (partly) crystalline systems Djayaprawira et al. (2005), Ikeda et al. (2008), Parkin et al. (2004), and Yuasa et al. (2004). 


\section{Tunnelling anisotropic magnetoresistance}

Relatively recently Gould et al. (2004) first reported a new type of AMR in MTJs: tunnelling anisotropic magnetoresistance (TAMR). The novel effect occurs in devices with at least a single ferromagnetic layer and an insulating barrier. TAMR can be divided into two categories: out-of-plane TAMR, where the magnetisation is rotated within a plane perpendicular to the ferromagnetic layer, and in-plane TAMR. The main causes for TAMR are SOC effects due to asymmetries in the system and magnetisation dependent resonant tunnelling (Khan et al., 2008). We will focus on the specific example of out-of-plane TAMR due to Bychkov-Rashba SOC (Bychkov and Rashba, 1984).

At surfaces and interfaces the symmetry of a solid-state system is broken in the direction perpendicular to that surface or interface. This structural asymmetry leads to an asymmetry of the potential in the same direction. In the reference frame of the electron the electric field associated with this asymmetric potential produces an effective magnetic field that interacts with the magnetic moment of its spin. The effect leads to the following contribution to the Hamiltonian:

$$
\mathrm{H}_{\mathrm{BR}}=\alpha(\boldsymbol{\sigma} \times \mathbf{p}) \cdot \hat{z}=\left(-\alpha \hbar k_{y}, \alpha \hbar k_{x}, 0\right) \cdot \boldsymbol{\sigma} .
$$

The parameter $\alpha$ is the strength of the Rashba coupling; $\sigma$ the Pauli matrix vector. $p$ is the crystal momentum and equal to the product of Planck's constant divided by $2 \pi, \hbar$, and the wave vector $k$. The unit vector $\hat{z}$ points in the direction perpendicular to the surface or interface. The term within parenthesis on the right-hand side is called the spin orbit field, $\boldsymbol{w}_{\mathrm{BR}}$, and interacts with the spin of the electron like an effective magnetic field. In a system with a welldefined magnetisation direction, $\hat{\mathbf{M}}$, the energy shift for spin up and spin down electrons is (Matos-Abiague and Fabian, 2009)

$$
\Delta \mathrm{E}_{\uparrow, \downarrow}= \pm \boldsymbol{w}_{\mathrm{BR}} \cdot \hat{\mathbf{M}}
$$

By applying a large enough external magnetic field the orientation of the magnetisation, $\hat{M}$, can be changed causing anisotropic shifts in the electronic states at the interface or surface. These shifts 
can, for instance, effect the coupling between different states and influence the availability of resonant states leading to observable changes in the the transmission probabilities of the system (Chantis et al., 2007; Khan et al., 2008).

\subsection{INTERFACES IN SPINTRONICS}

The underlying mechanisms of TMR and TAMR rely on electronic states in the ferromagnetic layer, the tunnel barrier and at the interface. The MR ratios that can be achieved in these MTJ are governed by the coupling of these states to one another and are therefore very sensitive to interfacial parameters such as crystallinity and relative orientation of the layers, roughness and the presence of defects. Consequently, the fabrication of high quality interfaces is an active topic of research.

\section{Hybrid interfaces}

Within the field of organic spintronics one of the key topics is the injection of spin polarised current from a ferromagnetic metal into an organic semiconductor. Effects like hybridisation, charge transfer, broadening and shifts of molecular states strongly influence charge transport, and because the are typically spin dependent, also spin transport across the interface (Atodiresei et al., 2010, 2011; Barraud et al., 2010; Sanvito, 2010).

\section{Spin filtering at the nickel-graphene interface}

Karpan et al. (2007, 2008) predicted and Lazić et al. (2014) confirmed a spin filtering effect at interfaces between nickel and graphene and cobalt and graphene that bears some resemblance to TMR in crystalline systems. Transport in graphene is limited to the $\mathrm{K}$ points in reciprocal space, where the Dirac cones intersect with the Fermi level (Castro Neto et al., 2009). Because graphene and nickel (111) have a good lattice match, $<1.5 \%$, their in-plane Brillouin zones are nearly identical and the electronic states in nickel that 
can easily cross the interface into the graphene can be easily identified by an in-plane k-vector component at the $\mathrm{K}$ point. Due to the exchange splitting of the nickel DOS each spin orientation has different states available for transport near the Fermi level. And, as it happens, only the minority spin orientation has any states near the Fermi level with an in-plane $k$-vector component at the $\mathrm{K}$ point, leading to spin selective charge transport across the interface.

For a single layer of graphene, sandwiched between ferromagnetic leads, the spin filtering effect is annihilated due to hybridisation effects, leading to high tunnelling probabilities through the graphene for both majority and minority spin electrons. But Martin et al. $(2014,2015)$ showed that when a tunnel barrier is added a single layer of graphene already leads to reversal of the TMR. Moreover, the tunnelling current decreases exponentially with the number of graphene layers and for five or more graphene layers becomes negligible, resulting in perfect spin filtering. It is this prediction that formed the initial motivation for our investigation of device containing nickel-multilayer graphene interfaces. 


\subsection{INTRODUCTION}

To investigate magnetotransport properties of nickel-graphene interfaces, these have to be fabricated and subsequently incorporated in a measurement device. In this chapter we will first shortly discuss the process for growing multilayer graphene* on nickel and show some characterisation of these films.

The properties of the fabricated graphene and underlying nickel are strongly inhomogeneous, therefore, using the results from the characterisations, a method was found to select small areas with comparable and suitable film properties. Subsequently, this method was incorporated in the development of a fabrication process of spintronic devices containing nickel-graphene interfaces. A detailed overview of all process steps and the used parameters can be found in the back matter of this thesis.

The reason for developing a fabrication method that directly uses the wafer on which the graphene has been grown in stead of, for instance, transferring the graphene onto a magnetically well-defined substrate (Cobas et al., 2012), is the demand for a clean interface with a structural relation between the nickel and the graphene. Moreover, because of hybridisation effects that strongly modify the electronic structure of the first graphene layer and the need to suppress tunnelling through the graphene, we want to be able make devices that contain multiple layers of graphene.

* Throughout the remainder of this thesis the adjective multilayer is omitted and the term graphene is loosely used to also indicate a stack of several, typically no more than ten, layers of graphene. 


\subsection{MULTILAYER GRAPHENE ON NICKEL}

All the graphene that was used in this project has been produced using a chemical vapour deposition (CVD) process on nickel (Muñoz and Gómez-Aleixandre, 2013; Zhang et al., 2013). In this process a silicon wafer with a nickel top layer is heated to typically $90{ }^{\circ} \mathrm{C}$ to $1100{ }^{\circ} \mathrm{C}$ in a controlled environment, e. g., gas mixture or vacuum. A carbon containing precursor gas, in our case methane, flows over the sample and decomposes at the hot surface. The released carbon atoms diffuse into the nickel and segregate to the surface to form graphene when the wafer is subsequently cooled down. A nickel single crystal with (111) surface termination favours the formation of a monolayer of graphene, but step edges, grain boundaries in polycrystalline films and other defects promote the growth of localised patches of multilayer graphene (Zhang et al., 2010).

At the beginning of the project there was no possibility of producing our own graphene within the research group so we sourced two $10 \mathrm{~cm}$ wafers with CVD grown graphene on nickel from a commercial party ${ }^{\dagger}$. Although we do not know the exact parameters we expect the used growth process to be very similar to the one described above. Since the graphene layer is commonly transferred to another substrate with the sacrificial nickel layer chemically removed, the properties of the nickel are not specified by the manufacturer and probably also not controlled. This led us to perform some basic characterisations of the nickel layers in the commercially bought wafers.

Figure 3.1 shows the results of XPS analysis of the two commercial graphene wafers. By irradiating the sample with X-rays and recording the kinetic energy of emitted electrons XPS measures the elemental composition of the sample, and additionally provides information on the chemical states of the present elements through shifts in their binding energies. The carbon and oxygen peaks are attributed to surface contamination. The attenuation of these peaks

+ http://graphene-supermarket. com/One-wafer-100mm-Graphene-Film- on-Nickel. html 


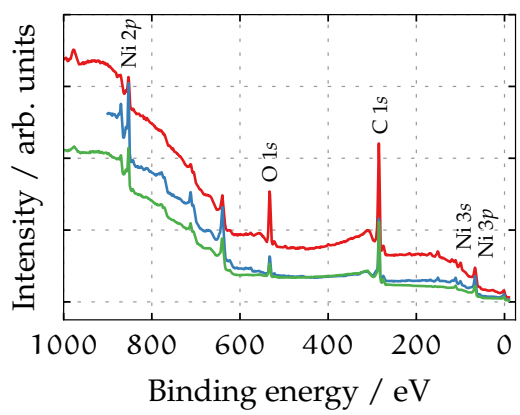

(a) Wide range scan.

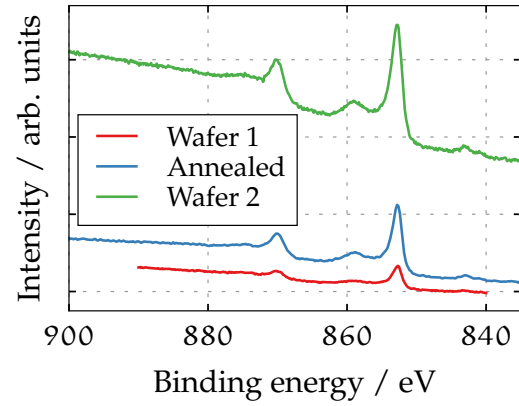

(b) Ni $2 p$ peak.

Figure 3.1: XPS spectra of the commercially bought nickel-graphene wafers show no signs of oxidation of the nickel layer. Wafer 1 has been measured before and after in situ annealing. The attenuation of the oxygen and carbon peaks after annealing support the assumption that these materials are present due to surface contamination.

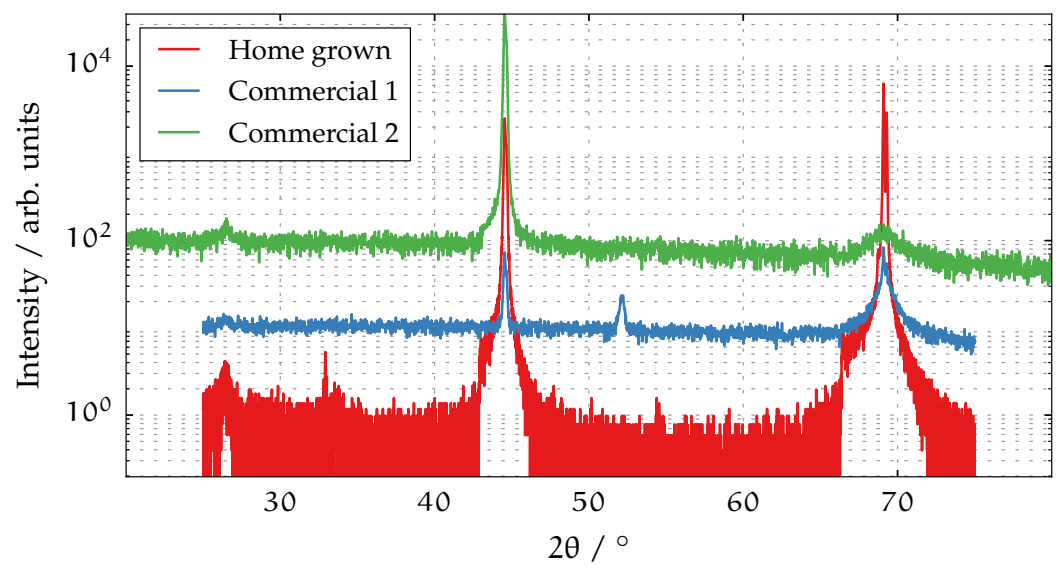

Figure 3.2: XRD spectra of the two commercially bought and one of our home grown nickel-graphene wafers show that the nickel films are not monocrystalline but do have a preferential (111) orientation. The peak around $45^{\circ}$ belongs to the nickel fcc (111) orientation; the one around $52^{\circ}$ to nickel fcc (200). At $69^{\circ}$ the silicon (400) peak from the substrate can be seen. (vertical offsets added for clarity.) 


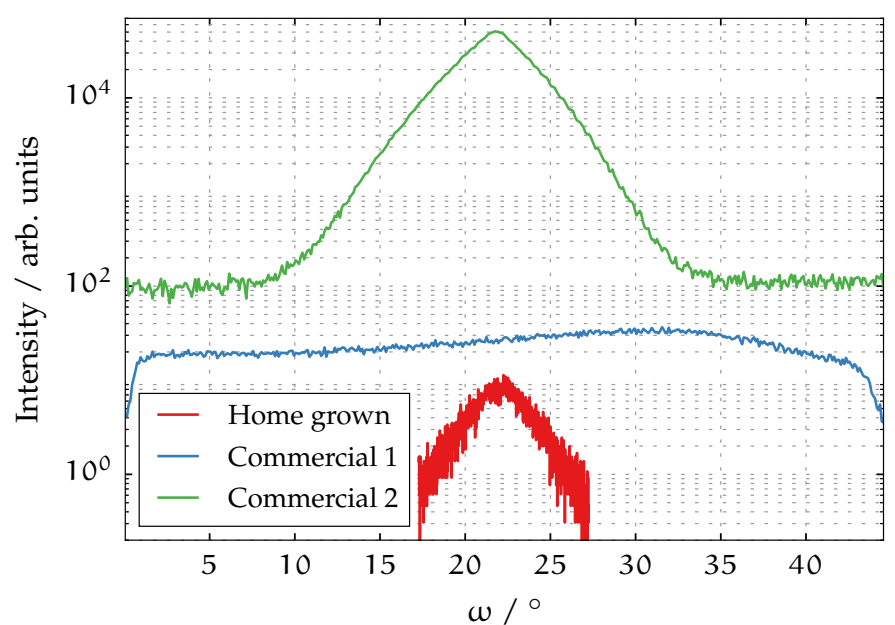

Figure 3.3: XRD rocking curves of the nickel fCC (111) peak. By tilting the sample while keeping the X-ray source and detector fixed the presence of tilted crystal grains can be investigated. The first commercially bought wafer shows intensity over the whole measurement ranges indicating a random orientation of the crystallites. The other two measurements show peaks indicating a preferred orientation with the fcc (111) plane towards the surface. (vertical offsets added for clarity.) 
after in situ annealing and the lack of a nickel oxide feature at the high binding energy side of the nickel $2 p$ peak support this idea.

XRD measurements provide insight into the crystallographic properties of the nickel layers (figure 3.2). Here a clear difference can be seen between the wafers. The first commercially bought wafer shows both a nickel (111) and a nickel (200) peak with relative intensities comparable to that of a nickel powder spectrum (See, for instance, Wang et al. (2006)). Combined with the lack of a peak in the rocking curve shown in figure 3.3 this indicates a random orientation of the nickel crystallites on this wafer. The measurements of the second commercially bought and one of our home grown wafers show a preferential orientation with the nickel (111) direction pointing out of the plane of the sample. A broad but clear peak in the rocking curves of both samples indicates that there is some relative tilt between the crystallites. These results suggest that the nickel layers of both commercially wafers are at different states of recrystallisation. Having the lowest surface energy, grains with a (111) orientation are the most favourable and grow while consuming grains with other orientations. For the second commercial and the home grown wafers this process seems to be more complete than for the first commercial wafer, possibly due to differences in process temperatures and/or durations.

For the home grown wafers first an approximately $200 \mathrm{~nm}$ thick nickel film is grown using physical vapour deposition (PVD) on a silicon/silicon oxide wafer. The wafer and film are heated up to $950^{\circ} \mathrm{C}$ in $15 \mathrm{~min}$ to improve crystallinity of the surface and remove volatile impurities, this annealing step is done in a 1 mbar hydrogen atmosphere believed to reduce the amount of some specific impurities and remove metal oxides through reduction (Muñoz and Gómez-Aleixandre, 2013). With the wafer still at $950^{\circ} \mathrm{C}$ methane gas is let into the chamber during $10 \mathrm{~min}$, while maintaining a pressure of 1 mbar and a methane flow rate of typically $10 \%$ of the hydrogen flow rate. Subsequently, the wafer is cooled down to $400{ }^{\circ} \mathrm{C}$ in $9 \mathrm{~min}$, and subsequently let to cool down in an atmosphere of pure argon at 5 mbar. 


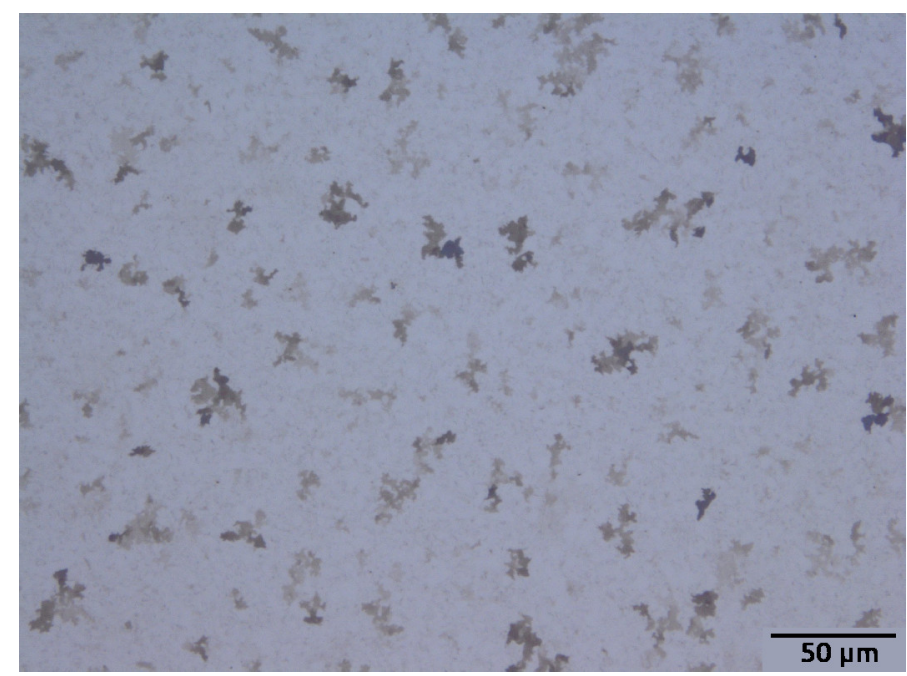

Figure 3.4: Optical image of a typical nickel-graphene sample. Dark patches, corresponding to multiple layers of graphene, can be seen scattered over the surface.

We continue with an investigation of the properties of both the commercially bought and the home grown graphene films. Figure 3.4 shows a typical optical microscope image of the surface of the nickel-graphene stack. The darker patches are areas where multiple layers of graphene are stacked on top of each other. To illustrate this we used a setup of HybriScan Technologies ${ }^{\ddagger}$ that combines an optical microscope, a SEM and Raman spectroscopy. Raman spectroscopy shows, among others, the vibrational modes of a system by illuminating the sample with monochromatic light and recording the scattered photons with shifted energies. The aforementioned setup offers the possibility to record data using all three techniques from the same area of the sample (figure 3.5). The recorded images in figures $3.5 \mathrm{a}$ and $3.5 \mathrm{~b}$ show that the darker patches in the optical image correspond one-to-one with darker patches in the SEM image. Since selection of the locations for Raman spectroscopy is done using the SEM image this makes it possible to easily correlate these locations with the recorded optical images.

$\ddagger \overline{\text { Registered Trademark. http://hybriscan.com (Timmermans et al., 2016) }}$ 


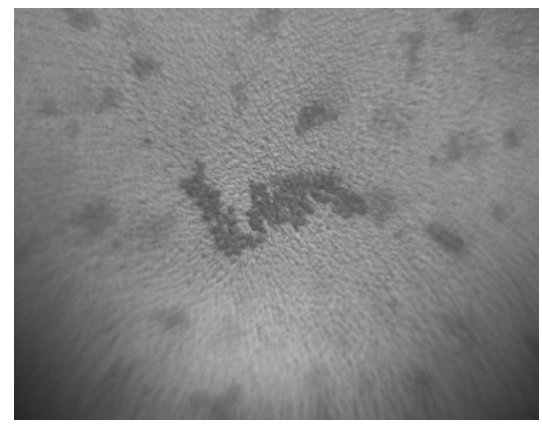

(a) Optical microscope.

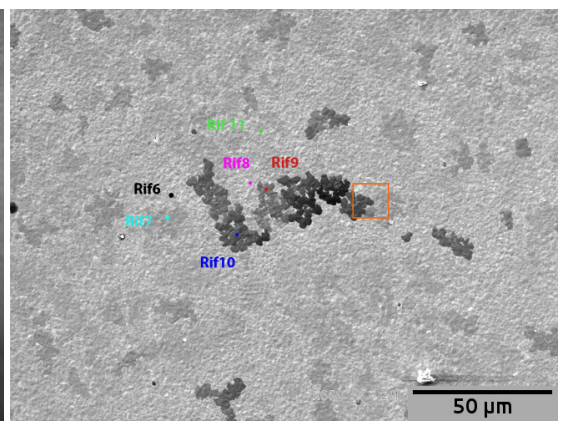

(b) SEM image.

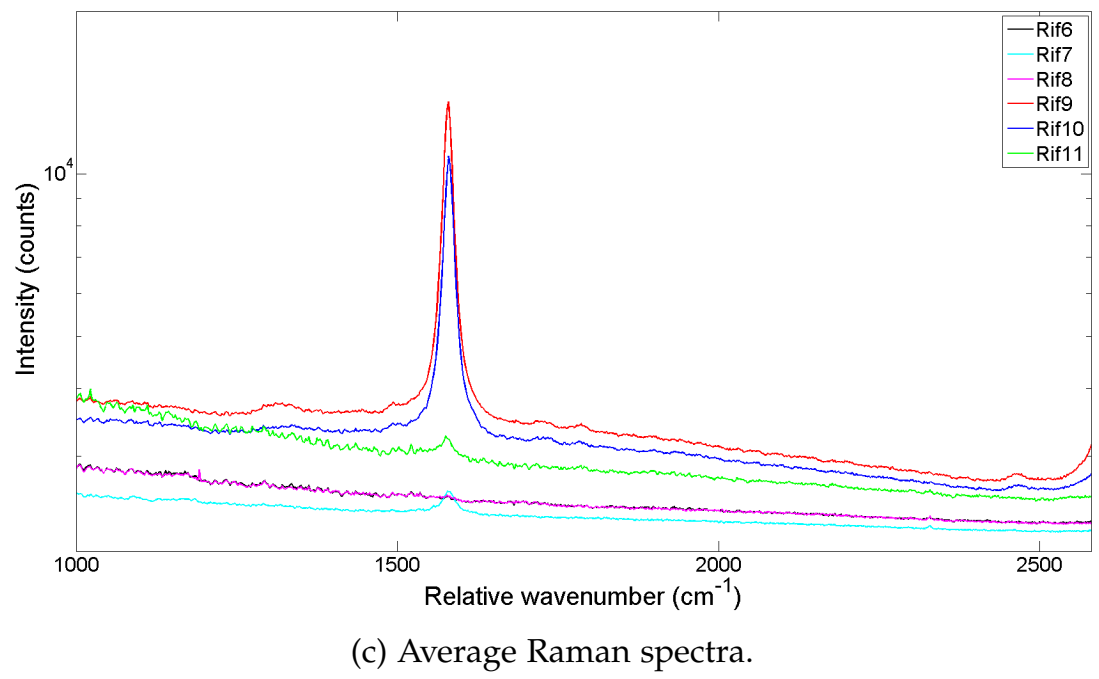

Figure 3.5: Correlated optical and SEM images and Raman spectra as recorded using the HybriScan setup. Dark patches in the optical images match with darker patches in the SEM image. The Raman spectra are taken at the locations indicated in the SEM image and show a correlation between the $G$ peak intensity and the darker coloured sample locations. For an actual estimate of the graphene thickness the scan should also include the $2 \mathrm{D}$ peak, this however lies outside the range of the used detector. (Measurements performed by Jelmer Boter.) 


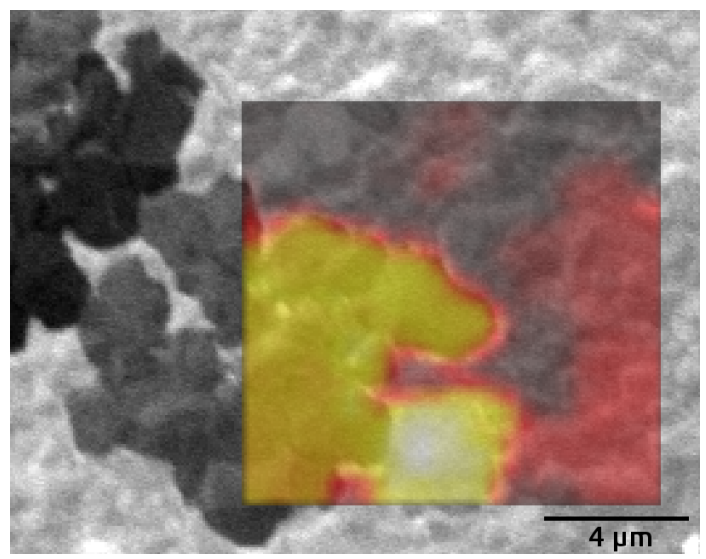

Figure 3.6: Combined image of SEM and spatial Raman data. The greyscale image is a SEM recording of part of the sample surface. The Raman data is shown in false colour with the colour scale representing the intensity of the Raman G peak. (Measurements performed by Jelmer Boter.)

By scanning over a selected area of the sample and recording the Raman spectrum at each point in a grid within this area a spatial Raman image can be recorded. In figure 3.6 the spatial Raman data is combined with the SEM data by overlaying the SEM image with the intensity of the graphene G peak (Ferrari et al., 2006) in the Raman spectrum at each location in false colour. Using this technique correlations between the datasets recorded using different measurement techniques can be found.

The spectral range of the Raman setup we used in the last examples only includes the graphene $G$ peak. To estimate the thickness of graphene using Raman spectroscopy both the $G$ and 2D peaks need to be recorded (Ferrari et al., 2006). By using a clustering algorithm the spatial Raman data can be grouped together in clusters with similar spectra. From these spectra the graphene thickness can be estimated by comparing the ratio of the $\mathrm{G}$ and $2 \mathrm{D}$ peak intensities and the shape of the 2D peak with literature (Ferrari et al., 2006; Nguyen et al., 2014). Our measurements show that the darker areas in the SEM images, and thus also the darker areas in the optical 


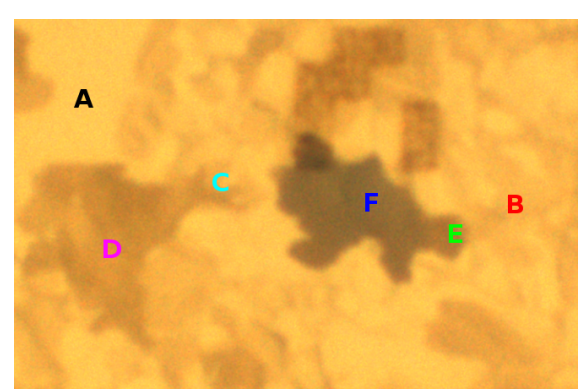

(a) Optical image.

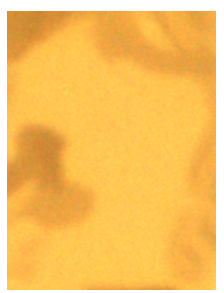

(c) 1 layer.

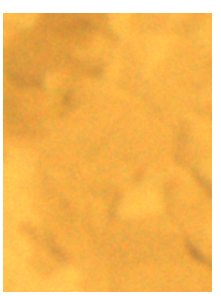

(d) 2 layers.

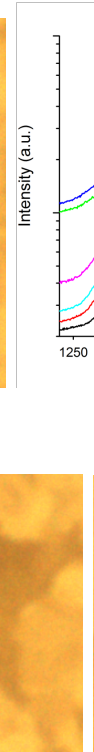

(e) 3 layers.

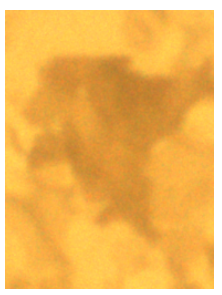

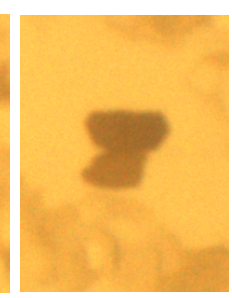

(f) 4 layers.

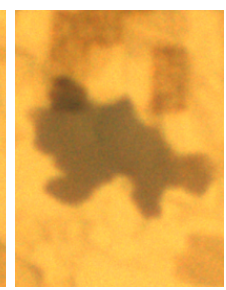

(g) 5 layers.

Figure 3.7: (a) Optical image of part of a graphene covered nickel layer showing areas with clearly distinct colours. (b) Raman spectra taken at the locations indicated by the characters in (a) and used to estimate the local graphene thickness. (c)-(g) Optical images of graphene patches with estimated number of graphene layers based on the Raman analysis from (a) and (b). The areas shown in images (c)-(g) are approximately $7 \mu \mathrm{m}$ to 1o $\mu \mathrm{m}$ wide.(Measurements performed by Jelmer Boter.) 


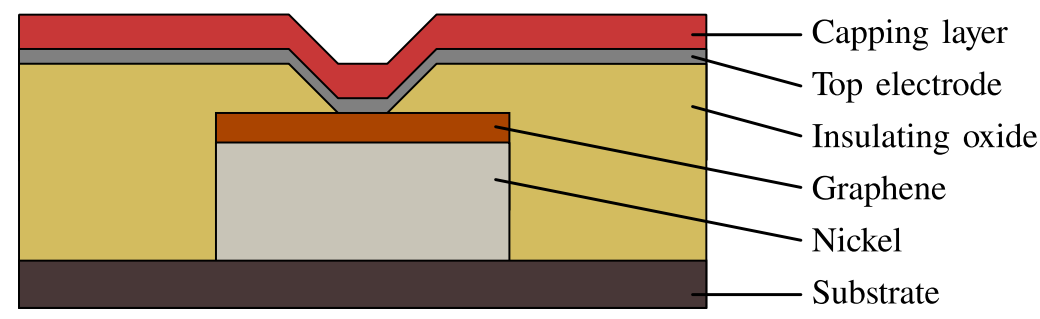

(a) Schematic cross section.

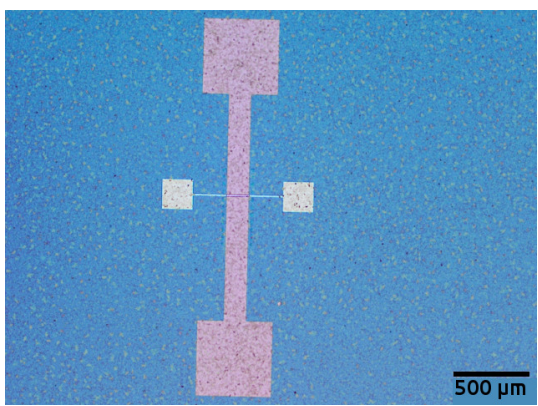

(b) Top view of actual device.

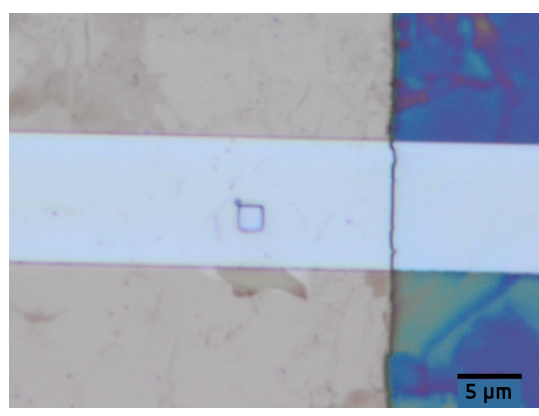

(c) Detail of contact hole.

Figure 3.8: Overview of the device layout. (a) Schematic cross section of the device. (b) Shows an optical microscope image of a finished devices. The large vertical structure is the bottom electrode defined in the nickel-graphene layer; the horizontal structure is the top electrode. The square pads at the end of the electrodes are for contacting. (c) Close-up image showing the contact hole.

microscopy images, correspond to clusters of thicker graphene. Figure 3.7 shows an example of the results of the cluster analysis and a overview of the estimated number of graphene layers. We are confident that we can rely on optical microscopy images for selecting suitable and thick graphene flakes. 

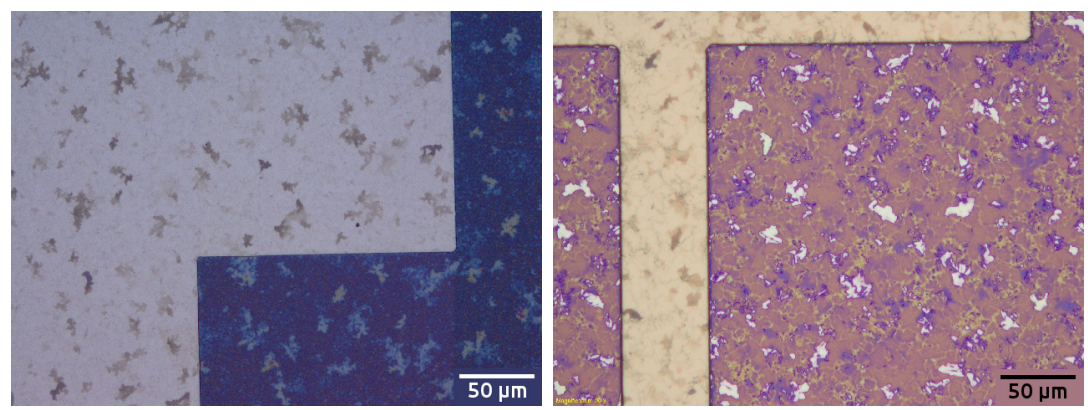

Figure 3.9: Optical images of the bottom electrodes defined in the nickelgraphene stacks by ion-beam etching. The images show residual patches with a metallic appearance in the etched away areas.

\subsection{DEVICE FABRICATION}

The goal of our devices is to measure the electrical transport in the direction perpendicular to the graphene-nickel interface, i.e., perpendicular to the sample surface. The device layout we designed to measure this is schematically shown in figure 3.8 together with microscope images of an actual device. It consists of two relatively large rectangular electrodes: A bottom electrode consisting of graphene covered nickel, and a metallic top electrode aligned at a right angle with the bottom electrode. The electrodes are separated by a thick insulating layer and are in contact over a small area where an opening has been created in the insulating layer. This contact hole is positioned on top of a selected graphene flake using microscopy and e-beam lithography, as explained further below. When a bias potential is applied between top and bottom electrode the current can only flow through this small contact hole. The choice of materials for the top electrode can be varied depending on the type of measurement to be done. Next we will present a chronological outline of the fabrication process and provide some additional background where needed.

We start out with a (piece of a) silicon wafer that is completely covered with a $200 \mathrm{~nm}$ to $300 \mathrm{~nm}$ thick nickel layer with a CVD 

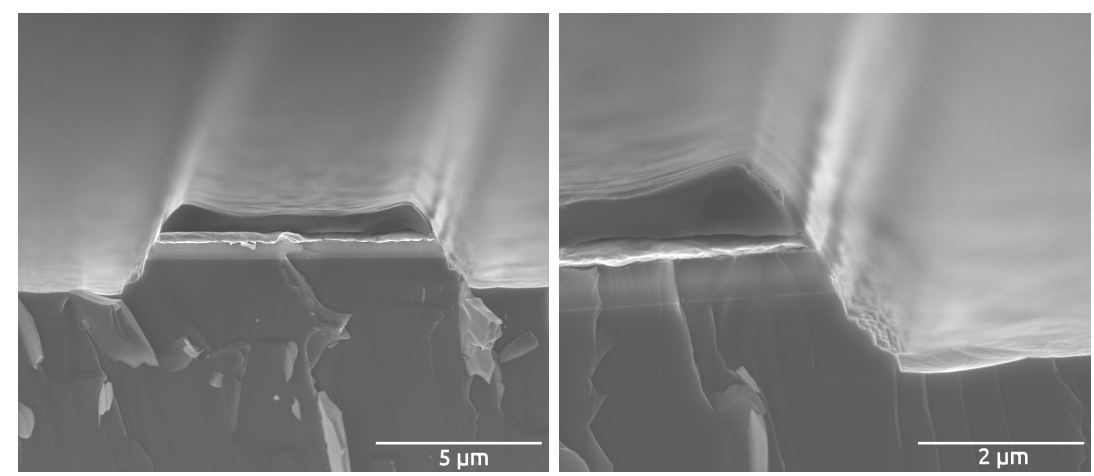

Figure 3.10: SEM images of etched bottom electrodes. The uneven layer on top of the electrode is the photoresist layer used for patterning, below that from top to bottom the stack consists of graphene (not visible), nickel, silicon oxide, and silicon.

grown graphene top layer. The shapes of the bottom electrodes are defined in a $1.7 \mu \mathrm{m}$ thick layer of optical photoresist (Olin OiR 90717) using photolithography and the nickel and graphene are etched away from the surrounding area using ion beam etching with argon ions. Even after long etching times, typically $55 \mathrm{~min}$, small patches of nickel seem to still be present on the surface (figure 3.9) and every sample has to be inspected to ensure these patches are well separated and cannot form an unwanted conduction path. At the edges of the etched bottom electrodes we have observed residual patched with outlines that perfectly align with the outlines of thicker graphene flakes on the electrodes. The thicker graphene has a much higher resilience to argon ion bombardment than the nickel (Williams et al., 2003) leading to large local differences in the final etching depth. The SEM images in figure 3.10 shows the effects of the long etching time on the profile of the edges of the electrode. The photoresist will also be severely damaged by the ion bombardment, but the image shows the remaining layer to be thick enough to have properly protected the surface of the electrodes.

After removal of the residual photoresist the whole sample is covered with an insulating layer of silicon oxide by magnetron sputtering. In earlier samples poly(methyl methacrylate) (PMMA) was 


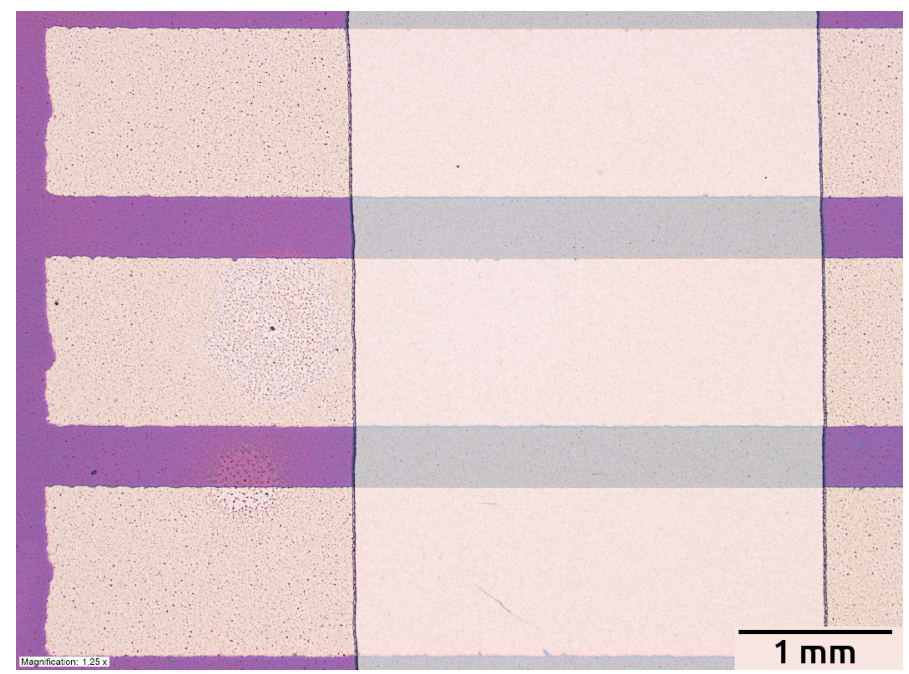

Figure 3.11: Microscope image of device used for testing the insulating layer. The darkest purplish areas show where the nickelgraphene stack has been etched away to define the bottom electrode (white vertical bar). The three horizontal bars are aluminium-copper top electrodes. 


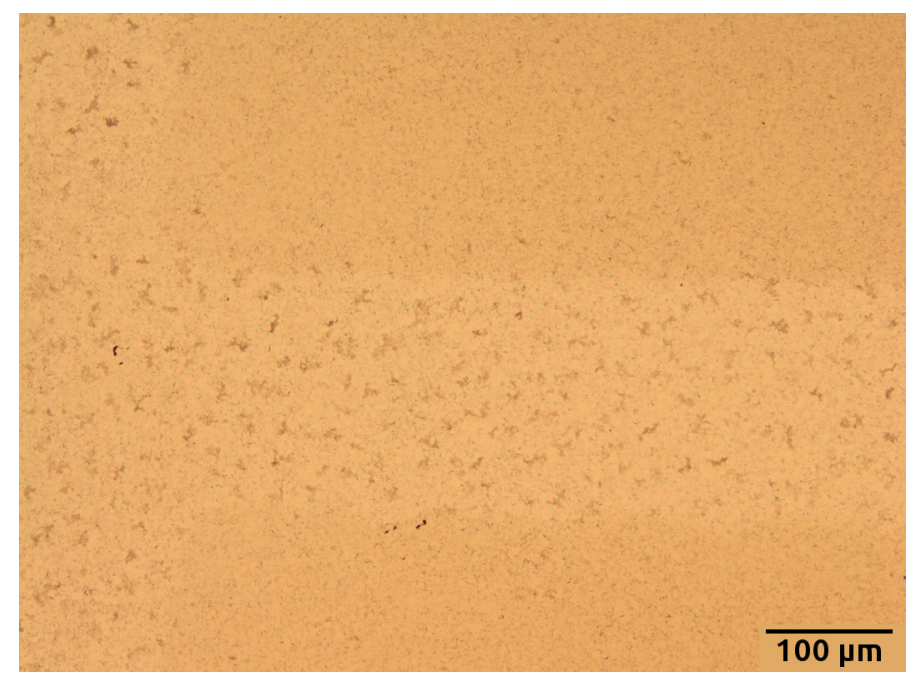

Figure 3.12: Damage to the graphene due to oxygen (ions) during the sputtering process. The more homogeneously coloured areas in the top and bottom of the image were exposed during the deposition. The patches of thicker graphene that are visible in the protected, unexposed area have disappeared.

used as an insulating layer since this can be patterned directly by photolithography and thus simplifies the fabrication process. However, these layers turned out to be unstable under the influence of temperature cycles, required for magnetotransport measurements at cryogenic temperatures, leading to high leakage currents through the film. To assess the insulating properties of the layer some tests were done with a simplified device layout. A single large bottom electrode was etched out of the nickel-graphene film on several samples and the samples were covered with oxide. Top contacts of $100 \mathrm{~nm}$ aluminium with a $10 \mathrm{~nm}$ copper capping layer were evaporated through a shadow mask to give the layout shown in figure 3.11. The so-fabricated plane capacitor devices should ideally show zero current under application of a DC bias voltage.

To get the best insulating properties the layer should be stoichiometric $\mathrm{SiO}_{2}$. Although the used deposition source is $\mathrm{SiO}_{2}$ we expect to get a substoichiometric oxide when using only argon for 


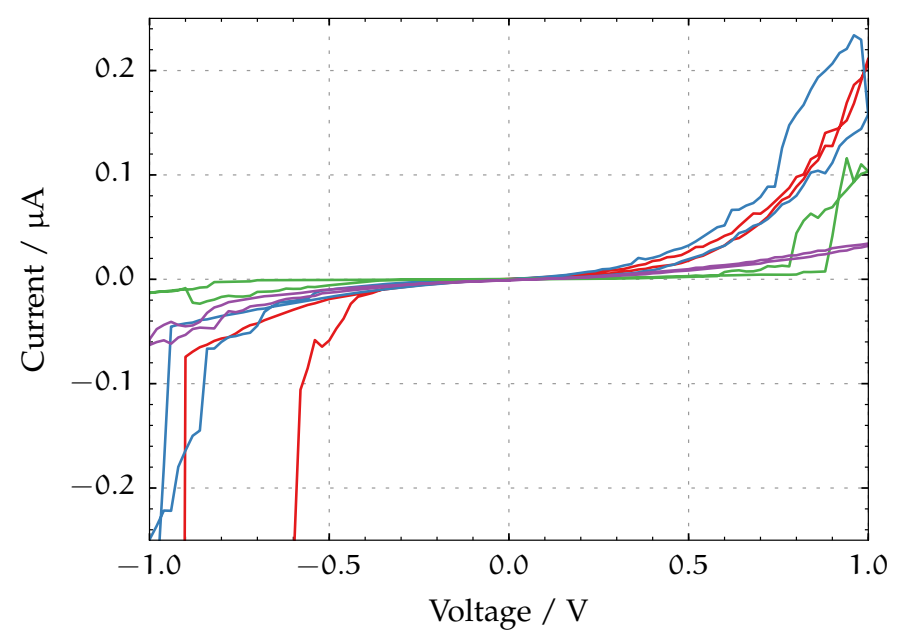

Figure 3.13: IV measurements for $100 \mathrm{~nm}$ thick oxide for four different junctions. The layer shows hysteretic behaviour and jumps in the resistance that occur at highly irreproducible bias voltages. Differential resistances around zero bias are up to $100 \mathrm{M} \Omega$. (Measurements performed by Remco Smits.)

the sputter process. To improve the quality of the oxide oxygen can be added to the chamber during sputtering, this was however found to lead to severe damaging of the graphene (figure 3.12). To both protect the graphene and get the best stoichiometry of the oxide layer in actual sample fabrication, the flow of oxygen into the sputter chamber is not switched on until a few minutes after the start of the deposition so the graphene will be protected by a few nanometers of oxide.

A thicker oxide layer will lead to steeper features at the edges of the contact hole that are not covered nicely by a thin top electrode. We therefore want to find out what the thinnest oxide layer is that shows good insulating properties. Several different thicknesses of the insulating layer were fabricated and characterised at room temperature. IV curves for the thinnest layers of $100 \mathrm{~nm}$ show hysteretic behaviour and switching between states with large differences in resistance (figure 3.13). Thicker layers of $150 \mathrm{~nm}$ and 


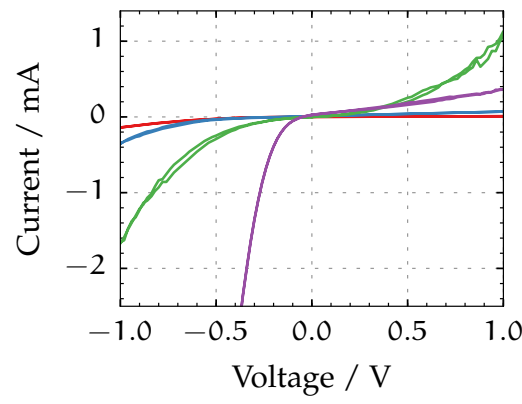

(a) Non-linear behaviour.

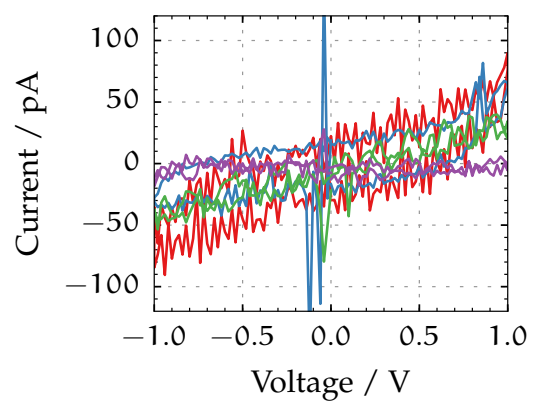

(b) "Good" junctions.

Figure 3.14: IV measurements of junctions with $150 \mathrm{~nm}$ and $200 \mathrm{~nm}$ thick oxide layers. (a) Curves for one junction with $150 \mathrm{~nm}$ oxide (red curve) and three with $200 \mathrm{~nm}$ oxide (blue, green and purple curves) that show non-linear behaviour. (b) Curves for two junctions with $150 \mathrm{~nm}$ oxide (red and blue) and two with $200 \mathrm{~nm}$ oxide (green and purple) that show satisfactory high resistance. (Measurements performed by Remco Smits.)

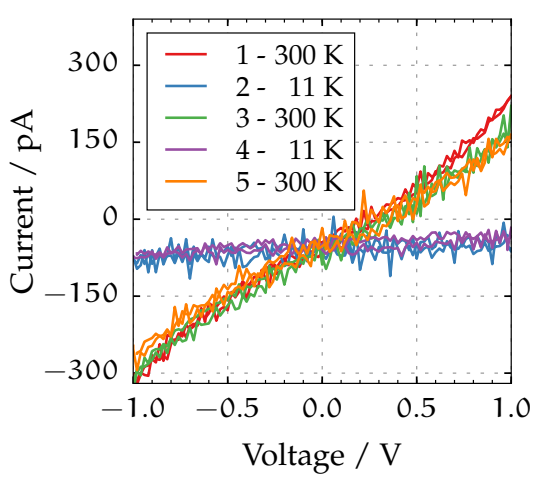

(a) Junction 1 .

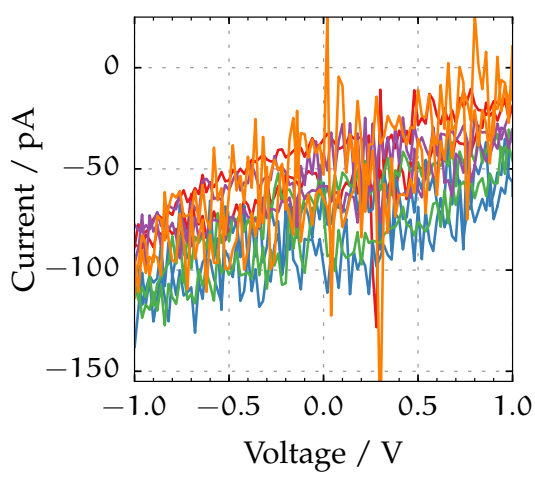

(b) Junction 2.

Figure 3.15: Thermal cycle tests. IV characteristics for two junctions first measured at room temperature, then at $11 \mathrm{~K}$, then again at room temperature, etc. No unstable behaviour due to repeated cooling down and heating up of the samples is observed. (Measurements performed by Remco Smits.) 
$200 \mathrm{~nm}$ show either very high resistances $\left(\gg_{1} G \Omega\right)$ as desired, or non-linear IV curves at a somewhat lower resistance (figure 3.14). Since none of the thicker layers seem to be completely insulating the decision was made to use a $200 \mathrm{~nm}$ thick layer and see whether the remaining conductance would vanish at the cryogenic temperatures used in actual experiments. The temperature dependent IV curves (figure 3.15) not only show satisfyingly high resistances at $11 \mathrm{~K}$, but also prove the stability of the insulating properties after subsequent cooling and heating cycles. Analysis of the IV characteristics at intermediate temperatures (not presented here) show an exponential relation between the conductance and the inverse temperature, suggesting that the decrease in resistance when approaching room temperature is caused by defect induced levels in the band gap of the oxide. Because the overlapping area of top and bottom electrodes in the actual devices is about 2400 times smaller than in these test structures, the observed finite leakage at low temperature is expected to have no significant impact on device performance.

The next step is to create the holes in the insulating layer where the top and bottom electrodes are in electrical contact. The $\mathrm{SiO}_{2}$ will be etched isotropically using buffered hydrofluoric acid (BHF) through a resist layer structured using electron beam lithography (EBL). We have already seen that we can use optical microscopy images to select the thicker multilayer graphene flakes. However, we also know that our nickel film is polycrystalline and that the growth of multilayer graphene flakes is catalysed by grain boundaries (figure 3.16). We noticed that by closing the condenser diaphragm of the optical microscope the surface topography of the nickel layer can be made visible (figure 3.17). AFM images of the same graphene flake show that the visible features do indeed match the surface structure (figure 3.18) and prove that we can rely on only optical microscope images to select locations with a flat surface and no grain boundaries underneath a thick graphene flake. One would preferably also ensure that the nickel surface has an (111) orientation, but available techniques such as ion channelling combined with backscatter electron detection will inevitably damage the surface. We therefore rely on the fact the recrystallisation favours the 


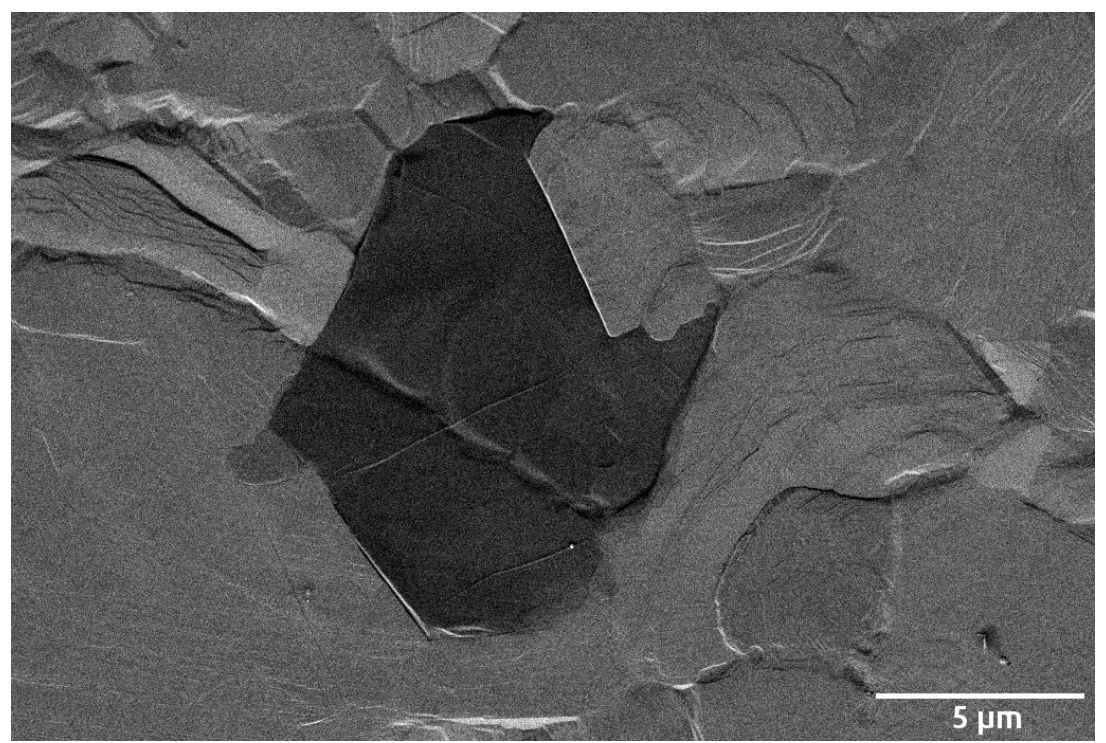

Figure 3.16: SEM image of a thick graphene flake on nickel. The surface structure of the nickel shows a clearly visible trench directly underneath the flake. Since during recrystallisation of the nickel layer grains with favourable orientations grow by absorbing surrounding material we expect these trenches to be grain boundaries. (Measurements performed by Kees van der Zouw.) 


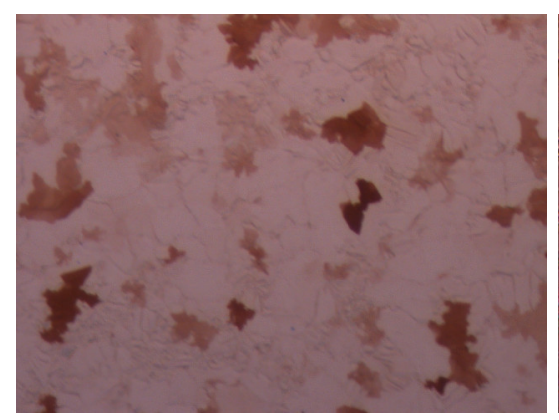

(a) "Normal" microscope image.

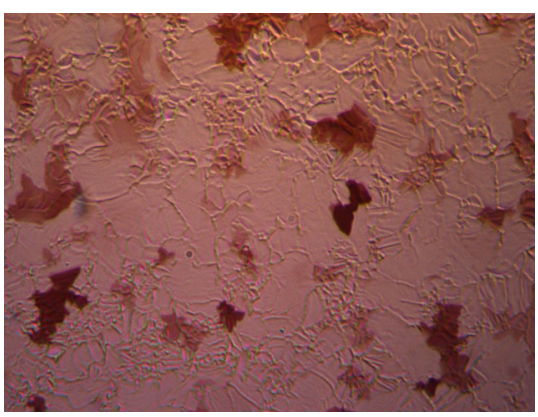

(b) Condenser diaphragm closed.

Figure 3.17: Optical microscope images of the nickel-graphene surface with (b) and without (a) contrast and depth-of-field enhancement by closing the condenser diaphragm. In (b) the surface structure of the underlying nickel film can be clearly distinguished.

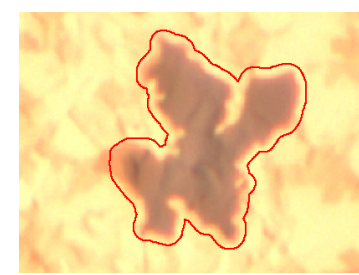

(a) Optical microscope.

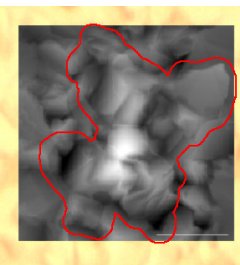

(b) AFM overlay.

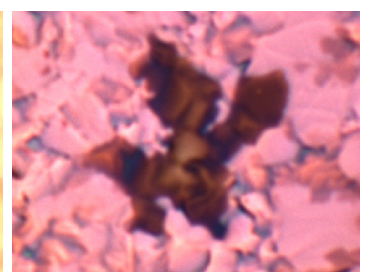

(c) Diaphragm closed.

Figure 3.18: One and the same graphene flake imaged with different methods. (a) "Normal" optical image of the selected flake. (b) An AFM image aligned with the optical image. (c) Optical image with enhancement of surface structure. The surface structure recorded by AFM matches that visible in the enhanced image. 


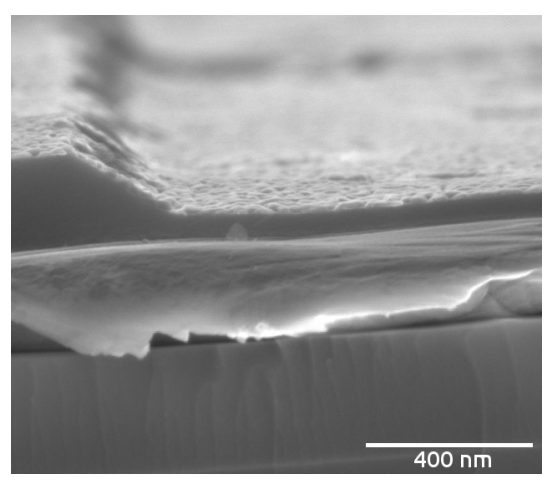

(a) 70 seconds.

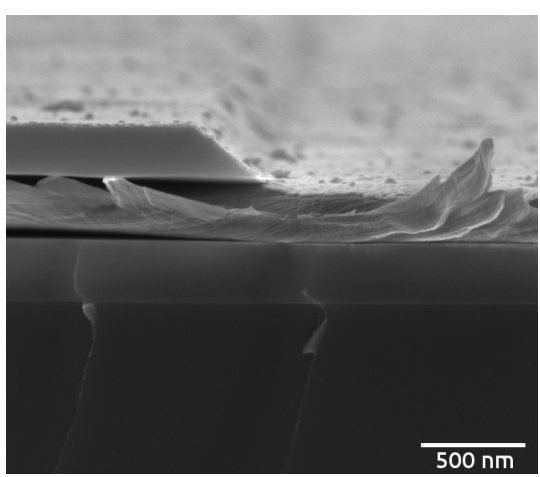

(b) 95 seconds.

Figure 3.19: SEM images of edges of holes etched in the oxide layer by isotropic BHF etching. The samples have been broken across the etched holes to take these images. In the left half of the images the oxide layer is still present. A layer of aluminium has been deposited on top of the oxide and hole to inspect the step edge covering. In (a) we can still see a layer of oxide in the etched area after $70 \mathrm{~s}$ of etching. In (b) the oxide layer has been completely etched away.

formation of a (111) surface due to its lower surface energy and assume the larger grains to most likely have this orientation.

The least reproducible step in the fabrication process is the actual etching of the hole in the oxide layer with BHF. An empirically determined rough estimate of the etch rate for our oxide is $2 \mathrm{~nm} / \mathrm{s}$ to $3 \mathrm{~nm} / \mathrm{s}$. Underestimation of the etching time leads to a residual oxide film in the holes (figure 3.19). Unfortunately, poor adhesion of the oxide layer on the graphene makes it possible for the BHF to flow in between the graphene and oxide. This leads to uncontrolled etching of the edges of the holes (figures 3.20a and 3.20b. Moreover, the oxide seems to separate from the graphene and buckle up around the hole leading to large steps, or even vertical gaps, at the edges of the hole as can be seen in the AFM images of figure 3.20. Attempts to counteract this delamination of the graphene by first depositing a thin (sub-monolayer) titanium layer Robinson et al. (2011) led to no improvements. 


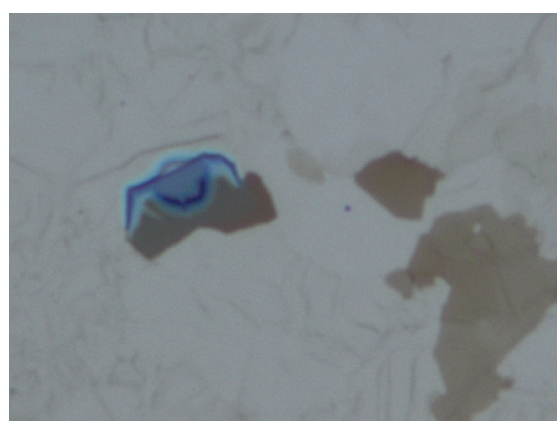

(a) Overetched hole.

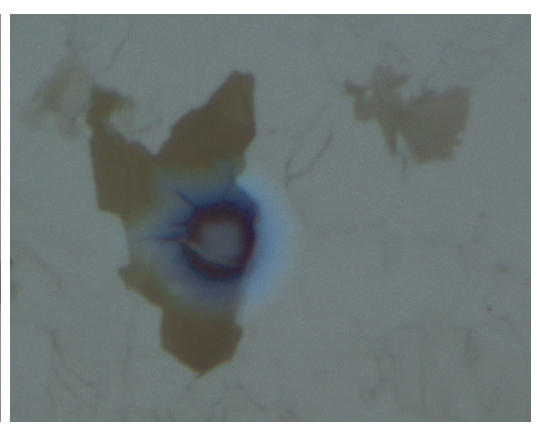

(b) Overetched hole.
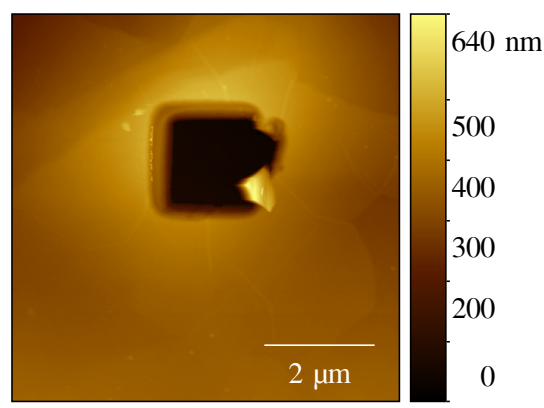

(c) AFM scan of a bad contact hole. (d) AFM scan of a good contact hole.

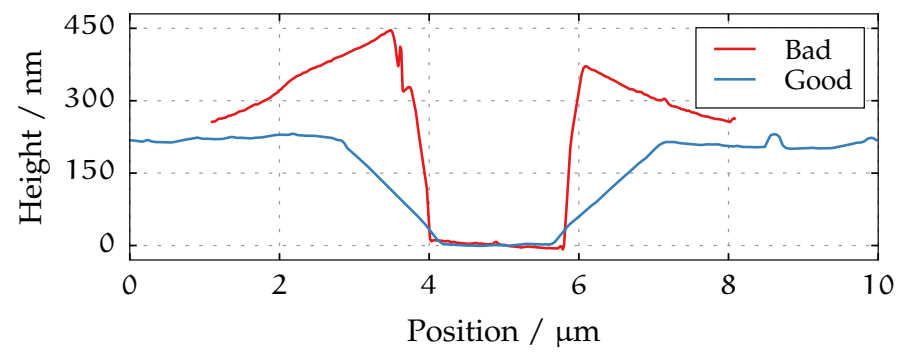

(e) Line profile comparison of AFM scans.

Figure 3.20: (a) and (b) show optical images of overetched holes with clearly visible damage of the oxide at the edges. The blueish discolouration of the oxide around the hole shows the separation of the oxide from the graphene. (c) shows an AFM image of an overetched hole. For comparison a "good" hole has been shown in (d). (e) shows a comparison of line scans taken horizontally through the centre of the holes in the AFM scans of (c) and (d). The height difference of about $450 \mathrm{~nm}$ between the edge and the bottom of the "bad" hole is more than twice the thickness of the oxide layer of $200 \mathrm{~nm}$. 


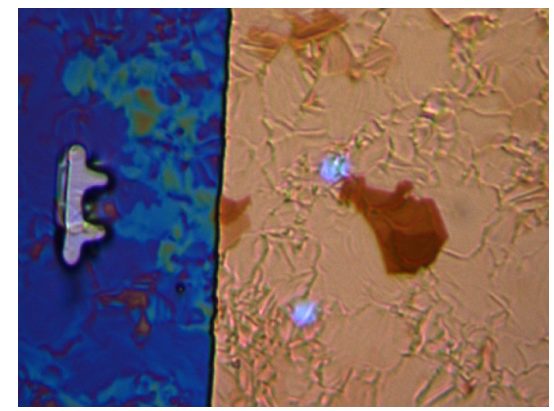

(a)

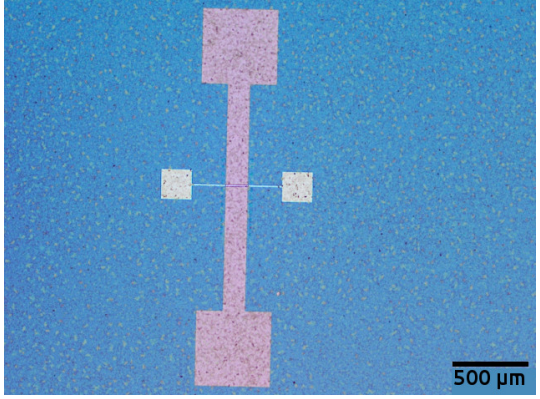

(c)

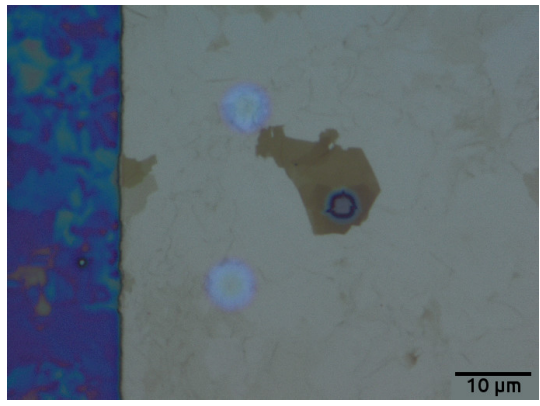

(b)

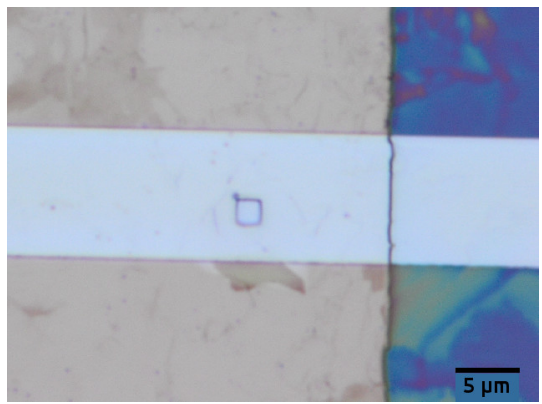

(d)

Figure 3.21: Images of a successful device fabrication. (a) The flake is selected by using the closed condenser diaphragm to show the surface topography underneath the graphene. This flake was chosen because of its dark colour, the hexagonal features of its edges and its position on a large nickel grain with highly probable (111) orientation (b) The hole in the oxide layer shows no signs of overetching and is nicely positioned on top of the graphene flake. (c) and (d) Images of a completed device (different actual device than (a) and (b). 
If all previous fabrication steps have been successful the top electrodes can be deposited by e-beam evaporation. The shape of the top electrodes is again written in a resist layer using EBL. The electrode material is deposited over the whole sample area and finally the resist is removed taking with it the top electrode material from unwanted locations. Figure 3.21 shows an overview of some of the steps in the fabrication of a device.

\subsection{CONCLUSION}

We have developed a fabrication method for devices containing a nickel-graphene interface that allows us to selective choose the position of our junction on the substrate. By comparing optical, Raman, AFM and SEM recordings we have shown that suitable locations, having a thick graphene flake on a large flat nickel grain, can be found using just optical techniques, greatly reducing the number of analysis steps involved in the process. Poor adhesion of overlayers on graphene, however, does pose a serious issue for achieving a good yield of properly functioning devices. 



\subsection{INTRODUCTION}

Using the fabrication scheme described in the previous chapter devices can be produced to investigate the electronic properties of the nickel-graphene interface. By varying the composition of the top electrode suitable devices can be produced for different kinds of transport measurements. In this chapter we will discuss some possible measurement types and the acquired results.

\subsection{SPIN FILTERING}

The first devices we made were intended for measurements to investigate spin filtering at the nickel-graphene interface as predicted by Karpan et al. (2007, 2008). Two possible ways of detecting the spin polarisation were explored: A spin valve method and a TedrowMeservey style measurement (Tedrow and Meservey, 1971, 1973). These two methods were chosen because they both provide a direct way of measuring the degree of spin polarisation in the current that passes through the device. The Tedrow-Meservey measurement requires a top electrode consisting of a tunnel barrier and an aluminium layer. The quality of the aluminium layer has to allow transition to a superconducting state. Moreover, it has to be flat and thin, $<10 \mathrm{~nm}$, in order for the superconducting state to withstand an in-plane magnetic field of several Tesla (Meservey and Tedrow, 1971). Several attempts were made to fabricate these samples but we never observed any signs of superconductivity, even without applied magnetic field. 


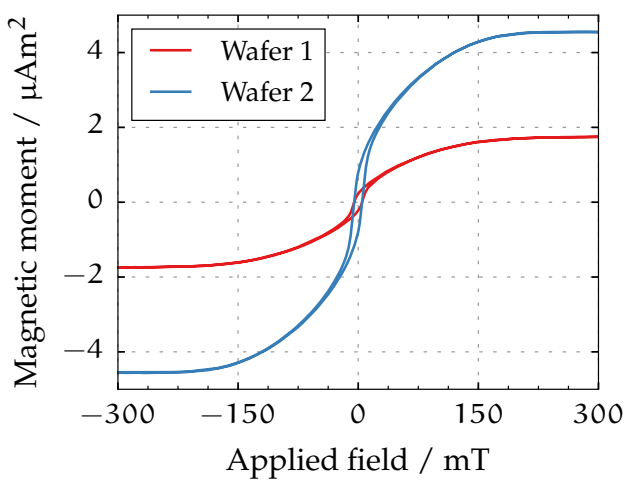

(a) Wide scan of magnetization.

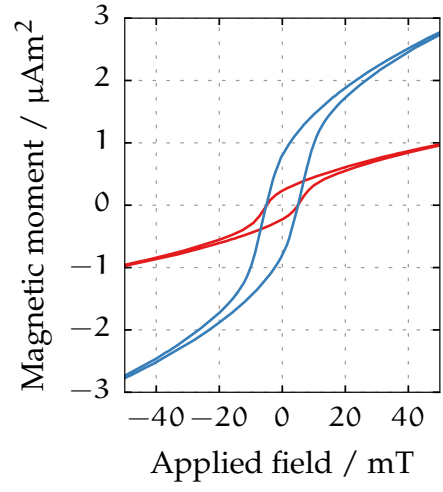

(b) Narrow scan.

Figure 4.1: Magnetisation curves of pieces of both commercially bought wafers with nickel-graphene layers measured by VSM. The external field is applied in-plane with the layer. The plotted magnetisation is in the same direction as the applied field. (b) Shows a zoom in on a smaller area around zero field. Both wafers show some hysteresis, but no sharp switching and the magnetisation saturates only gradually with increasing field. Estimates of the nickel layer thicknesses based on these measurements are $137 \mathrm{~nm}$ and $356 \mathrm{~nm}$ for "wafer 1" and "wafer 2", respectively. 
For a spin valve measurement the top electrode has to be made from a ferromagnetic material. Ideally, both electrodes should show sharp switching behaviour of the magnetisation direction at different coercive fields with a high remanent magnetisation in order to have well-defined parallel and anti-parallel magnetisation configurations. We investigate whether bottom electrodes that meet these requirements can be fabricated from the nickel layer that was used to grow the graphene. Using a VSM setup the magnetisation of the nickel-graphene film under applied magnetic field was measured (figure 4.1). In this technique a sample is placed on a vibrating holder inside a magnetic field. The current induced in pick-up coils placed around the sample holder by the moving sample is compared with that of a known reference sample to determine its magnetisation. Although the nickel films on both wafers show switching behaviour with a coercive field of $5 \mathrm{mT}$, the magnetisation only increases gradually with the external field and saturates just above $200 \mathrm{mT}$. This behaviour would be expected for a polycrystalline material with random orientations of the magnetocrystalline anisotropy. Some grains will have an "easy" axis roughly parallel to the direction of the externally applied magnetic field, these grains will have a high remanent magnetisation and a sharp switch of their magnetisation at the coercive field strength. For other grains the external field will be applied nearly parallel to a "hard" axis, the magnetisation of these grains will only be gradually turned away from an "easy" axes and saturates at higher field strengths. From the saturation magnetisation an estimate of the film thickness can be made. For a sample area of $25 \mathrm{~mm}^{2}$ and a bulk saturation magnetisation of nickel of $510 \mathrm{kA} / \mathrm{m}^{*}$ we find film thicknesses of $137 \mathrm{~nm}$ and $356 \mathrm{~nm}$ for "wafer 1 " and "wafer 2", respectively. This is quite a big difference that can't only be attributed to deviations in the sizes of the measured samples due to inaccuracy of breaking the wafer. These results seem to confirm again that not much care is given to the quality and exact thickness of the, in most applications sacrificial, nickel layer.

* Values from "Properties of Magnetic Materials," in CRC Handbook of Chemistry and Physics, 97th Edition (Internet Version 2017), W. M. Haynes, ed., CRC Press/Taylor \& Francis, Boca Raton, FL. 


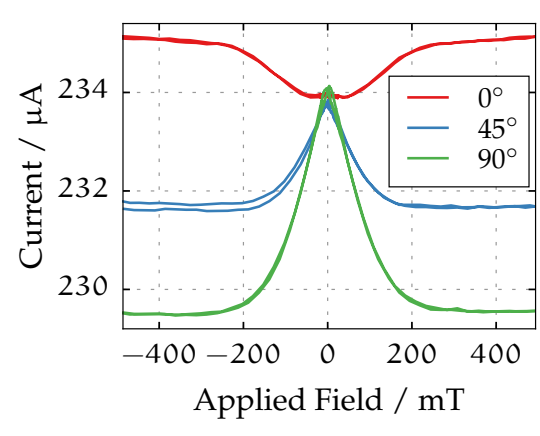

(a) Room temperature AMR.

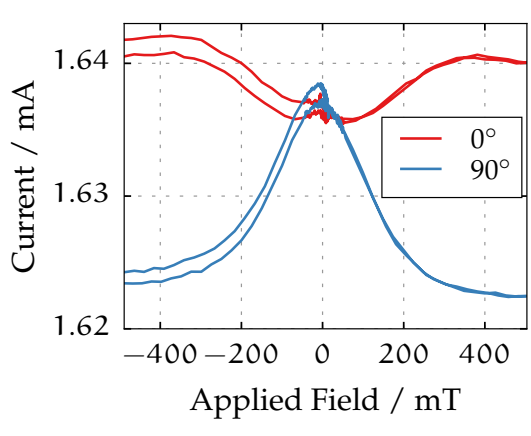

(b) AMR at $5 \mathrm{~K}$

Figure 4.2: AMR measurement of a bottom electrode etched from the nickel-graphene layer. All curves are recorded with a bias voltage of $10 \mathrm{mV}$. The magnetic field is always applied in-plane with the sample surface and the indicated angles are with respect to the direction perpendicular to the long axis of the bottom electrode, and thus also perpendicular to the direction of current flow. (Measurements performed by Kees van der Zouw.)

In our actual device design the aspect ratio of the bottom electrode, $60 \mu \mathrm{m}$ wide and $6 \mathrm{~mm}$ long, might introduce shape anisotropy and consequently improve the magnetic switching behaviour. In the ideal case we would make the bottom electrode as narrow as possible, just wide enough to accommodate the contact hole in the insulating layer. But since the bottom electrodes are defined with a mask that is aligned relative to the sample edges and thus can not be positioned freely, this would greatly reduce the probability of the bottom electrodes actually containing a suitable graphene flake on their surface. We measure the AMR of our samples by applying a constant bias of $10 \mathrm{mV}$ and measuring the current while changing the strength of the applied magnetic and repeating this for several different angles of the applied field with respect to the sample, and thus current direction. The AMR images in figure 4.2 show no improvement in the magnetisation behaviour of the electrodes. The curves exhibit very little hysteresis and very gradual saturation of the resistance, corresponding to saturation of the magnetisation in the direction of applied field. The fact that the 
curve with the external field applied perpendicular to the current direction shows the least change around zero field even shows that the remanent magnetisation does not point along the long axis of the bottom electrode. From the saturated resistance values at angles of $0^{\circ}$ and $90^{\circ}$, using equation 2.1 we find that the TAMR ratio $\approx \frac{2.35-2.295}{2.35} \approx 2.3 \%$, which is in good agreement with values reported in literature (McGuire and Potter, 1975).

Although the situation is less than ideal, it might still be possible to observe a spin valve effect, albeit with imperfect parallel and anti-parallel alignment of the electrode magnetisations, as long as the nickel directly underneath the contact hole exhibits a single magnetic domain with suitable magnetic switching behaviour. The magnetic domains of the nickel layer are visualized using MFM measurements (figure 4.3) (Martin and Wickramasinghe, 1987; Sáenz et al., 1987). The height image shows some surface features that are thought to indicate the outline of the crystal grains. The magnetic domains in the MFM image are serpentine-like and their dimensions much smaller than the estimated size of the nickel grains based on the surface topography. The images show no discernible correlation of the magnetic domains with the locations of nickel grains. In figures $4.3 \mathrm{C}$ and $4.3 \mathrm{~d}$ the MFM scans of an area of the nickelgraphene layer are shown with and without an applied external field of $10 \mathrm{mT}$. Some subtle shifts in the positions of domain walls can be made out, but nothing resembling switching of a complete domain. An unsurprising result when comparing the applied field of $10 \mathrm{mT}$, the maximum attainable in the used setup, with the magnetisation curves in figure 4.1.

The observation of magnetic domains that are smaller than the junction dimensions and the lack of homogeneously magnetised and switching domains render the successful formation of a spin valve highly infeasible. The use of much narrower bottom electrodes, potentially also with a thinner nickel layer, could improve the magnetic properties of the bottom electrodes considerably. The required modifications to the fabrication process to realize this are however not straightforward. Because of the less stringent requirements for the electrodes the choice was made at this point to focus our attention on TAMR measurements. 


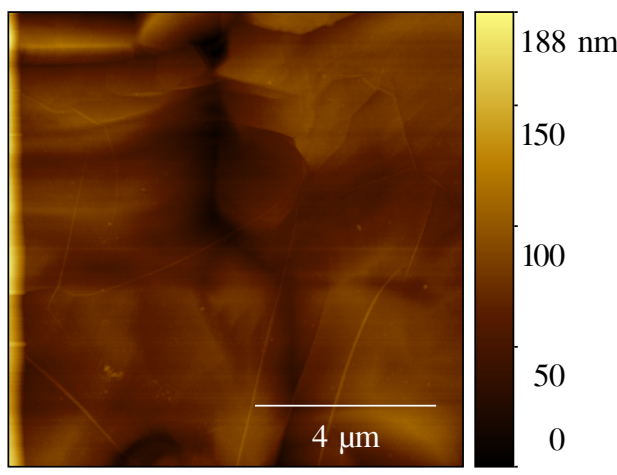

(a) Surface height.

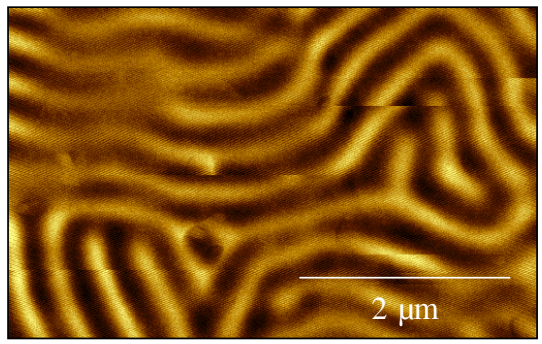

(c) Without external field.

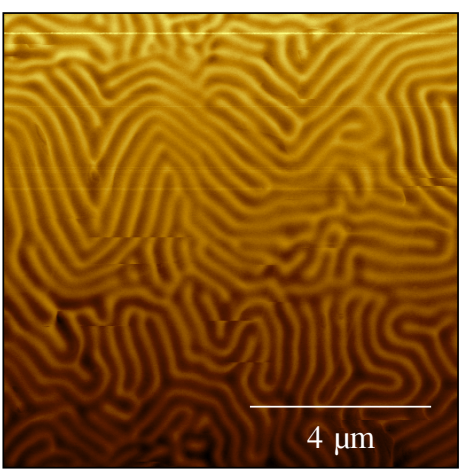

(b) Magnetic domain contrast.

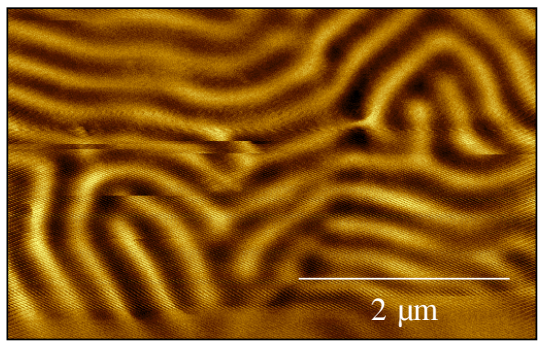

(d) With external field.

Figure 4.3: MFM scans of different areas of the nickel-graphene layer. (a) and (b) are taken on the same location and show separate height and magnetic domain information. (c) and (d) Show the magnetic domain contrast of the same area with and without an external applied magnetic field of $10 \mathrm{mT}$ (different location on sample than (a) and (b)). (Measurements performed by Kees van der Zouw.) 


\subsection{TUNNELLING ANisotropic MAgNetoresistanCE}

The following measurements are performed in a cryostat that can cool our sample down to below ${ }_{5} \mathrm{~K}$. A magnetic field of up to $9 \mathrm{~T}$ can be applied and the sample can be manually rotated around one axis from an orientation with the magnetic field perpendicular to the sample surface to an orientation with the field in-plane with the sample surface.

For obtaining the IV curves a four-point measurement technique is used: current is sourced from one end of the top electrode to one end of the bottom electrode and the voltage is measured between the opposite end of the top electrode and the opposite end of the bottom electrode. By using this method contact resistances are eliminated from the measurements. An added benefit, since we have wires connected to opposite ends of our electrodes, is that we can easily check for defects in or bad contact to the electrodes by performing a conventional two-wire measurement through the individual top or bottom electrode. Typical resistance values for the individual electrodes lie in the range $5 \Omega$ to $40 \Omega$.

All IV measurements were performed by first ramping the bias current from zero to the lowest, negative, value. Subsequently four full measurements were taken, two with increasing bias and two with decreasing bias, before ramping the current back to zero. The curves of the four individual sweeps are compared to inspect the stability of the measurement and to ensure the absence of hysteresis due to capacitive effects in the setup. All presented IV curves and those used for calculation of derived quantities are the averages of the four sweeps.

Figure 4.4 shows room temperature measurements of the transport through a device with a top electrode of $20 \mathrm{~nm}$ of aluminium capped with $10 \mathrm{~nm}$ of Copper. The junction shows ohmic behaviour with a resistance of $73 \mathrm{k} \Omega$. Aluminium was chosen as top electrode material because the lack of sharp features in its density of states around the Fermi level (Levinson et al., 1983) that could be falsely attributed to the nickel-graphene interface. 


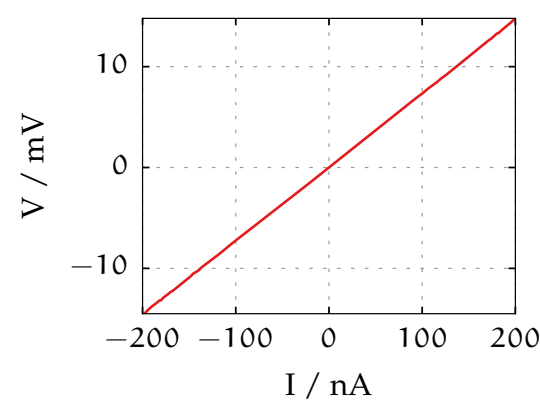

(a) Four point IV.

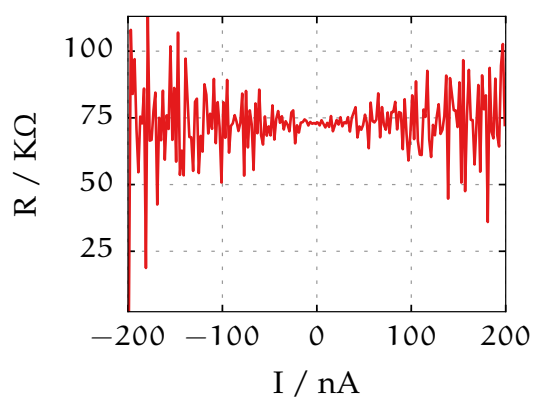

(b) Differential resistance

Figure 4.4: Four-point IV measurement and calculated differential resistance of a device at room temperature. The top electrode consists of $20 \mathrm{~nm}$ aluminium capped with $10 \mathrm{~nm}$ of Copper. The device shows linear ohmic transport with a resistance of $73 \mathrm{k} \Omega$.

The curves in figure $4.5^{\mathrm{a}}$ show transport measurements of the same device cooled down to $10 \mathrm{~K}$ and with an applied external field of $9 \mathrm{~T}$. The resistance at $10 \mathrm{~K}$ is no longer constant and varies between twice and four times the value at room temperature. We expect that the graphene acts as a tunnel barrier with very low barrier height that is not visible at room temperature due to the thermal broadening of features in the IV. At room temperature additional conduction is also possible at the sloping edges of the contact hole where the oxide layer is very thin. There is statistically significant difference between the IV curve with the magnetic field aligned perpendicular to the surface and the curve with the field parallel to the surface. Both the IV curves in figure $4.5^{\mathrm{a}}$ and the differential resistance curves in figure $4.5 \mathrm{C}$ can be divided in two regimes: In the range $-25 \mathrm{nA}$ to $25 \mathrm{nA}$ the resistance curve shows a plateau indicating ohmic conductance; outside this range we observe non-linear behaviour.

Based on the predictions by Karpan et al. $(2007,2008)$ we believe that these results can be explained by the coexistence of two transport modes in our device. For electrons with $k$-vectors away from the $\mathrm{K}$ points the graphene acts as a low tunnel barrier, consistent with the non-linear IV at higher biases (Simmons, 1963). Around 


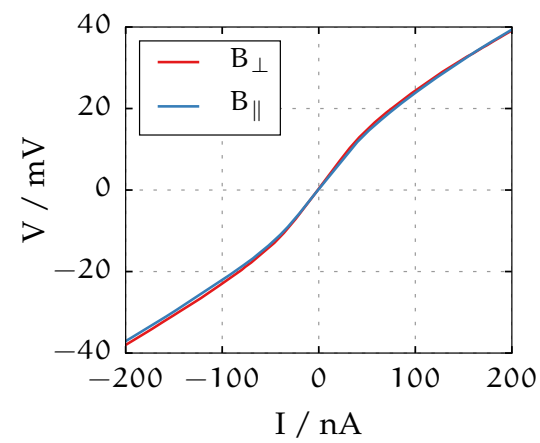

(a) IV curve.

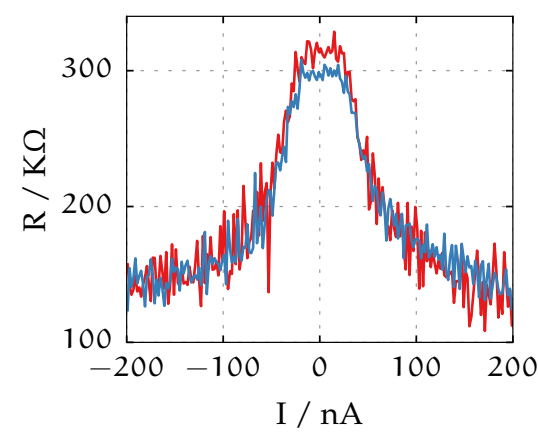

(c) Resistance.

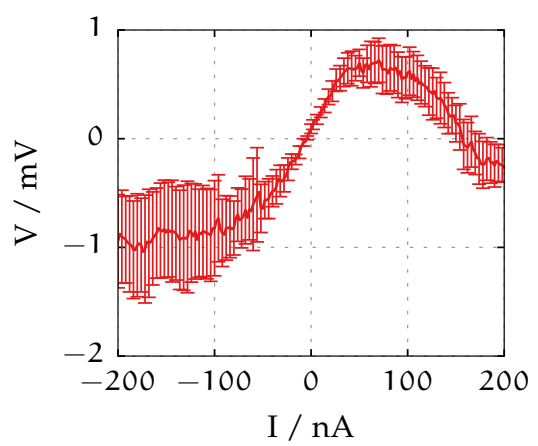

(b) Difference between IV curves.

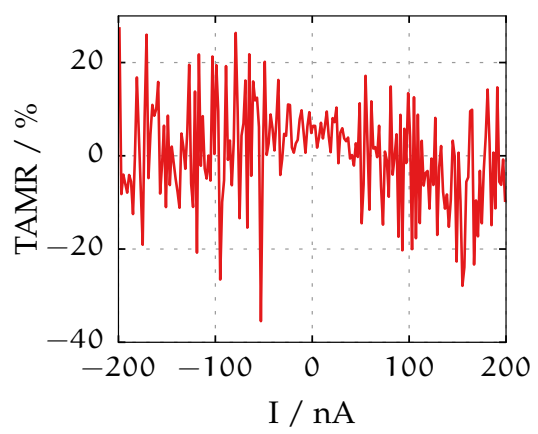

(d) TAMR ratio.

Figure 4.5: Transport measurements of a junction at $10 \mathrm{~K}$ with an applied external magnetic field of $9 \mathrm{~T}$ either perpendicular to the sample surface and thus the nickel-graphene interface (red) or parallel to the surface and interface (blue). The IV curves in (a) are the average of four consecutive measurements. (b) Shows the difference between the two measurements with error bars indicating the standard deviation calculated from the standard deviation of the averaging procedure. (c) and (d) are calculated from (a) 
zero bias the conduction is dominated by electrons with $k$-vectors near the $\mathrm{K}$ point that can flow via electronic states near the Dirac points with the same $k$-vectors in graphene. In this latter regime we observe a TAMR ratio of about $5 \%$ (figure $4 \cdot 5 \mathrm{~d}$ ).

Figure 4.6 show the TAMR measurements of a second device at ${ }_{5} \mathrm{~K}$. The measured resistances in this device are much lower than in the previous sample. The exact number of graphene layers can vary from device to device and influence the resistance, the exact size of the contact hole in the oxide layer can also be different, but neither of these effects can explain the observed difference in resistance of several orders of magnitude. A more likely explanation is a difference in contact area between the aluminium of the top contact and the graphene. These last samples have a top contact with $100 \mathrm{~nm}$ aluminium in stead of $20 \mathrm{~nm}$. In the SEM images of figure 3.19 it can be seen that the aluminium top layer looks grainy and for thin layers this could lead to discontinuities within the layer. Additionally, one of the main challenges in the sample fabrication is the adhesion between the graphene and layers directly on top of the graphene. Bad adhesion could lead to bad electrical contact for parts of the contact hole, and thus greatly reduce the effective device area. The possibility of the higher resistance in the previously discussed sample being due to a residual oxide layer between the graphene and aluminium is highly unlikely since we regularly observe overetched contact holes with the same fabrication parameters.

The measurements for different magnetisation directions show significant changes in the resistance over the complete measurement range, with TAMR ratios approaching $50 \%$. This value is much higher than the values of a few percent commonly reported for other systems (Gao et al., 2007; Grünewald et al., 2011), and also more than the $12.5 \%$ reported by Park et al. (2008) in a system with strong SOC. Chantis et al. (2007) have predicted TAMR ratios of up to $20 \%$ in a iron-vacuum-copper system attributed to the Rashba shift of interface resonant states. The large TAMR ration in our system can be caused by the strong Rashba SOC in the nickel-graphene system (Dedkov et al., 2008). The observed effect could be enhanced by the $k$-vector filtering in our system. Commonly, the largest con- 


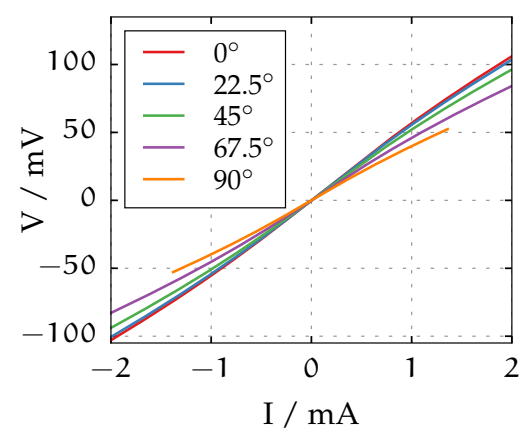

(a) IV curve.

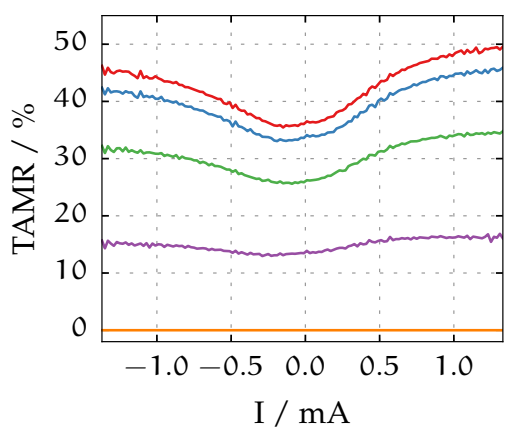

(c) TAMR ratio.

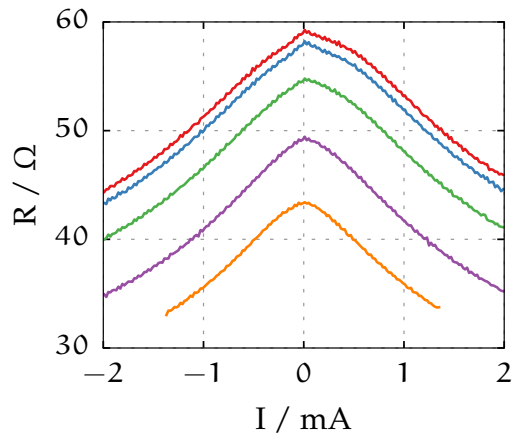

(b) Differential resistance.

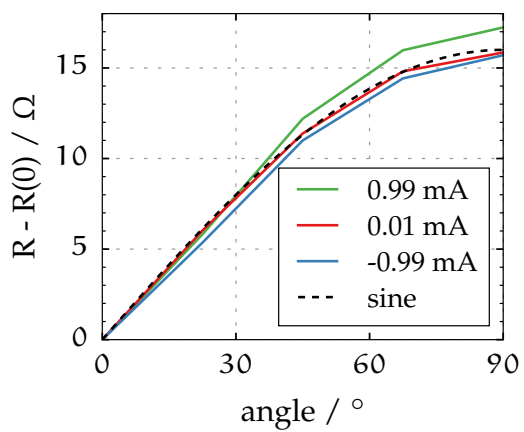

(d) Angle dependent absolute resistance change.

Figure 4.6: Transport measurements of a different junction measured at $5 \mathrm{~K}$ with an applied external magnetic field of $9 \mathrm{~T}$. The values given in the legend in (a) also apply to (b) and (c), and indicate the angle between the direction of the magnetic field and the direction normal to the sample surface and thus also the nickelgraphene interface. (d) shows the absolute change in resistance due to the rotation of the field at a fixed bias current. The measured values for a bias of $0.01 \mathrm{~mA}$ are in excellent agreement with a sine function with matching amplitude at $90^{\circ}$. 
tribution to a tunnel current is from electrons with a $k$-vector close to the $\Gamma$ point, because these experience the shortest effective tunnel distance. In our system there is a relatively large contribution from states around the $\mathrm{K}$ point that have a larger in-plane wave vector component and therefore experience a stronger Rashba effect. This suggest that the wave factor filtering effect leads to a larger TAMR ratio.

The angular dependence of the absolute change in differential resistance in figure $4.6 \mathrm{~d}$ was obtained by subtracting the resistance at $o^{\circ}$ from the resistance at other angles for identical values of the bias current. The curves for different biases are very similar in shape and differ at most $2 \Omega$ at $90^{\circ}$, also for bias currents not plotted in this figure. The shape of the curves is in excellent agreement with a sine function with amplitude equal to the absolute difference in resistance at $0^{\circ}$ and $90^{\circ}$ for a single bias current. The sine plotted in figure $4.6 \mathrm{~d}$ is fitted to the curve for a $0.01 \mathrm{~mA}$ bias current.

\section{$4 \cdot 4$ CONCLUSION}

Summarizing, the lack of magnetic domains with dimensions larger than our junctions and sharp ferromagnetic magnetisation reversal shows that the realisation of a spin valve with our nickel-graphene layers requires a considerable overhaul of the fabrication process. Measurements of devices with an aluminium top electrode show TAMR ratios approaching $50 \%$ with a sinusoidal dependence on the angle between interface normal and the magnetisation. The large observed effect, when compared to those reported for other systems, is attributed to the strong Rashba SOC in the nickel-graphene system and an enhancement of the TAMR ratio by the $k$-vector filtering. The observation of a linear resistance regime for small biases and a tunnelling regime for higher biases can be explained by selective band transport for only a subset of available carriers. 
SOLID STATE EPITAXIAL GROWTH OF NICOB ON GRAPHITE

\subsection{INTRODUCTION}

The fabrication of high-quality epitaxial interfaces is a topic of active interest within the field of spintronics. Very high TMR ratios are reported in MTJs with monocrystalline and epitaxial layers (Djayaprawira et al., 2005; Ikeda et al., 2008; Parkin et al., 2004; Yuasa et al., 2004). The measured TMR ratios exceed the theoretical maxima based on simple tunnel barrier models and confirm models that show the tunnelling conductance in epitaxial systems to be strongly dependent on the symmetry of the involved charge carrier states (Butler et al., 2001; Mathon and Umerski, 2001). Secondly, the spin filtering in cobalt-graphene-cobalt and nickel-graphene-nickel stacks investigated by Karpan et al. $(2007,2008)$ is only predicted to be perfect if all layers are epitaxial.

Previous work done in our research group by Wong et al. (2013) has shown a method to fabricate crystalline CoFe films on top of highly-oriented pyrolytic graphite (HOPG) by solid state epitaxy. The annealing in vacuum of an initially amorphous CoFeB alloy layer results in a polycrystalline film with a preferred orientation with the bcc (110) surface at the interface. During this process the boron segregates to the surface and the resulting film consists of ferromagnetic CoFe. While this is a strong proof of concept, the resulting bcc (110) surface does not share the hexagonal symmetry of graphite and has an overall poorer lattice match. In this chapter we investigate whether replacement of the initial $\mathrm{CoFeB}$ alloy in this process with $\mathrm{NiCoB}$ leads to the formation of a crystalline ferromagnetic layer with preferential fcc (111) orientation. 


\subsection{EXPERIMENTAL DETAILS}

Cobalt adopts a bcc structure only when grown as a thin films on specific substrates (Liu and Singh, 1993; Prinz, 1985). The formation of a bcc crystal phase in the work of Wong et al. (2013) is therefore attributed to the presence of iron in the alloy. To encourage the formation of a fcc crystal phase we investigate the possibility of using a nickel rich alloy without iron, NiCoB.

Lee et al. (2007) showed that the crystallisation process of amorphous $\mathrm{CoFeB}$ depends on the composition of the alloy. By depositing our $\mathrm{NiCoB}$ alloy through simultaneous plasma sputtering from three sources we can control the stoichiometry by varying the parameters of the individual sources. Unfortunately, the comparatively slow deposition rate of boron turned out to be a limiting factor to the range of variation.

The sputtering is performed in a vacuum system with a base pressure below $1 \times 10^{-6} \mathrm{mbar}$. The plasma power used for the reported data are $200 \mathrm{~W} \mathrm{RF}, 25 \mathrm{~W} \mathrm{DC}$, and $100 \mathrm{~W} \mathrm{DC}$, for respectively the sources of boron, cobalt, and nickel. The plasma guns are operated in an argon atmosphere at a working pressure of $7 \times 10^{-3} \mathrm{mbar}$.

Figure 5.1 shows X-ray reflectivity (XRF) measurements on three separate films of pure boron, cobalt and nickel on silicon acquired after 60 min of deposition for boron and cobalt and 30 min for nickel, using the same parameters as for deposition of the alloy. An X-ray beam is aimed at the sample at a grazing angle $\theta$ with the surface, and a detector measures the intensity of the X-rays reflected by the sample at the same angle $\theta$. The oscillations in intensity with varying $\theta$ show the back and forth change between positive and negative interference conditions of X-rays reflected off the surface of the sample and off the interface between substrate and deposited layer. From the period of these oscillations we find, using Bragg's law (Bragg and Bragg, 1913), that the boron, cobalt and nickel films have thicknesses of respectively $16 \mathrm{~nm}, 28 \mathrm{~nm}$ and $68 \mathrm{~nm}$.

From these thicknesses the relative amount of each element in an alloy produced from simultaneous co-sputtering of all three materi- 


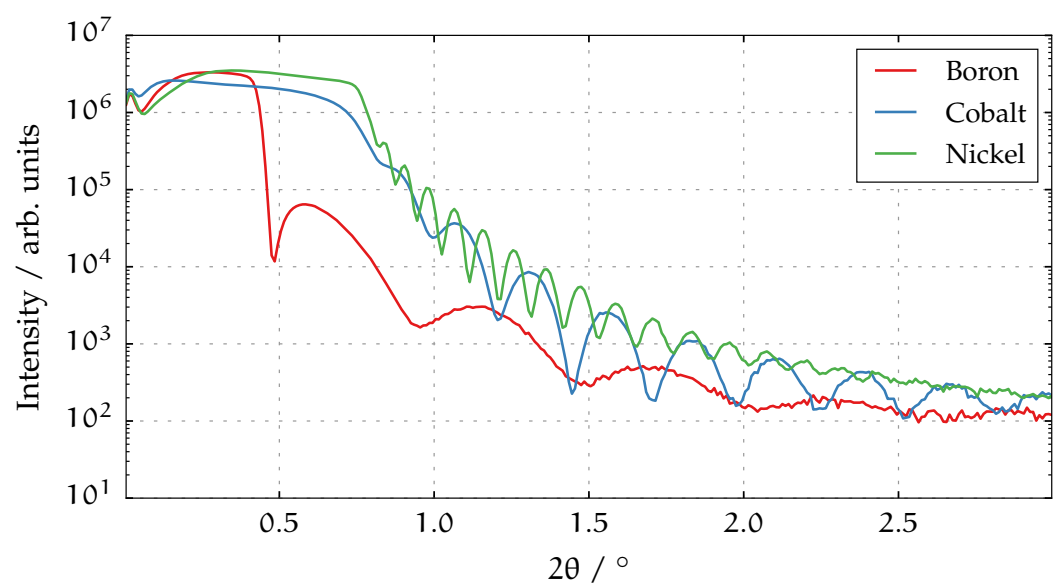

Figure 5.1: XRF curves for deposited pure boron, cobalt and nickel layers on a silicon wafer. From the period of oscillations the calculated film thicknesses are $16 \mathrm{~nm}, 28 \mathrm{~nm}$ and $68 \mathrm{~nm}$, respectively.

als can be calculated. The ratio of the specific gravity to the atomic weight of each element can be used to convert the film thicknesses to a value proportional to the number of atoms:*

$$
\begin{aligned}
\mathrm{Ni}: \mathrm{Co}: \mathrm{B} & =68 \cdot 2\left(\frac{58.7}{8.9}\right): 28\left(\frac{58.9}{8.9}\right): 16\left(\frac{10.8}{2.3}\right) \\
& \approx 78 \%: 16 \%: 6 \%
\end{aligned}
$$

The factor of 2 in the nickel term corrects for the fact that the deposition time for the nickel film is half that of the cobalt and boron.

In contrast to the CoFeB layers, that were grown from a single source alloy using e-beam evaporation in an ultra-high vacuum system that allows in situ annealing at temperatures up to at least $600{ }^{\circ} \mathrm{C}$, the $\mathrm{NiCoB}$ layers were initially grown from three separate

* Values from "The Elements," in CRC Handbook of Chemistry and Physics, 97th Edition (Internet Version 2017), W. M. Haynes, ed., CRC Press/Taylor \& Francis, Boca Raton, FL. 


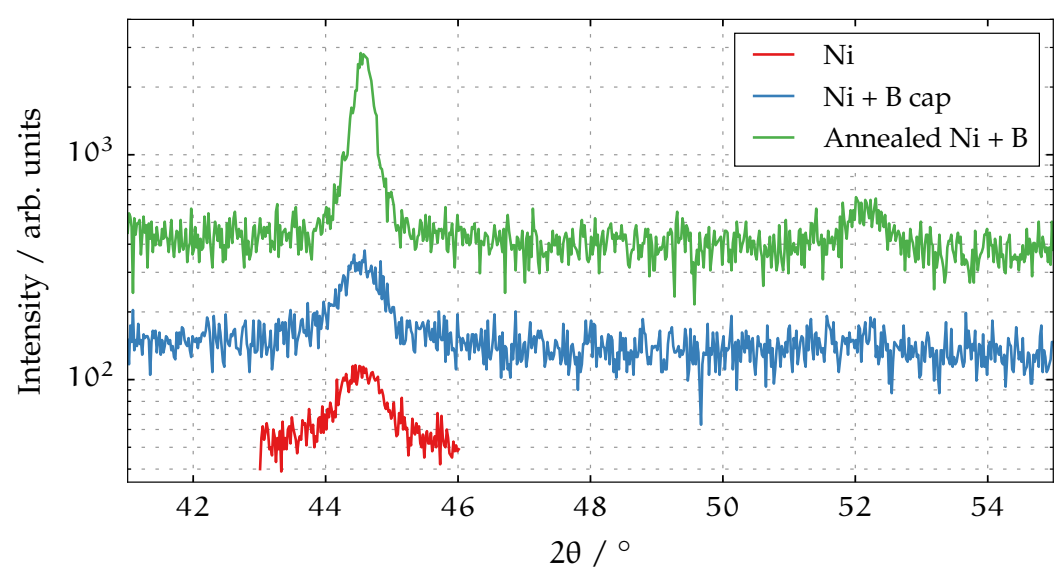

Figure 5.2: XRD measurements of as-deposited $68 \mathrm{~nm}$ nickel films with and without $4 \mathrm{~nm}$ protective boron layer, and a similar boron capped nickel layer after annealing for $2.5 \mathrm{~h}$ at $500{ }^{\circ} \mathrm{C}$ (added vertical offset for clarity). The peak at $44.5^{\circ}$ matches the nickel fCC (III) orientation. After annealing the intensity of this peak has increased and a small nickel (0O2) peak appears near $52^{\circ}$.

targets using magnetron sputtering in a different system. By using three separate targets the composition of the deposited film can be controlled and varied. This system did not offer the possibility of heating the sample, so the samples had to be taken out of the vacuum to be transferred to a tube oven for annealing under a nitrogen atmosphere. Exposure to air led to the expected problem of oxidation and after annealing most of the films contained dark discolourations or peeled away from the substrate in green transparent flakes. XPS analysis showed high oxygen, and sometimes carbon, content and both nickel oxide and boron oxide features.

To protect the layers against oxidation during transfer a capping layer of pure boron is deposited on top of the alloy. The influence of a $4 \mathrm{~nm}$ thick boron layer on top of a $68 \mathrm{~nm}$ pure nickel layer is investigated by XRD to test for negative side effects (figure 5.2). For larger values of the angle of incidence $\theta$ the peaks in the measured intensity correspond to constructive interference of X-rays scattered from atoms in different atomic planes, providing insight into the 
crystallographic properties of the sample. The peaks corresponding to the fcc (111) orientation of nickel are similar in shape and height for the as-deposited nickel with and without boron top layer. The higher intensity of the nickel (111) peak and the appearance of a nickel (002) peak for the sample annealed at $500{ }^{\circ} \mathrm{C}$ for $2.5 \mathrm{~h}$ suggest that the crystallisation of the nickel is not influenced by the additional boron. Unless specified otherwise all films reported on hereafter are covered with a $4 \mathrm{~nm}$ protective boron layer.

\section{$5 \cdot 3$ RESULTS}

Figure 5.3 shows XRD scans of the nickel fCC (111) peak for Ni, $\mathrm{NiCo}$ and NiCoB films on silicon before and after $2.5 \mathrm{~h}$ annealing at $500{ }^{\circ} \mathrm{C}$. For the nickel and $\mathrm{NiCoB}$ films the $\theta$ range around the nickel fcc (OO2) peak was also scanned (not shown here), only the annealed nickel film shows the appearance of a very small peak around $52^{\circ}$, about 1.5 times the background intensity. The absence, or very low maximum intensity for the annealed nickel film, of a (002) peak shows that crystallisation of the films leads to a preferential (111) surface terminated orientation. The intensity of the (111) peak is highest for the pure nickel film, showing it to have the highest crystallinity. Before annealing the peak of the NiCo is only slightly higher than that of $\mathrm{NiCoB}$, showing little effect from the added boron on the crystallinity of the deposited film. After the annealing the increase in crystallinity for the NiCo is stronger than for the NiCoB alloy.

To investigate the effect of the boron on the crystallinity of the film, we deposited a film with a higher boron content. Since the previous films were already deposited at the maximum power setting for the boron source, $200 \mathrm{~W}$ RF, we reduced the deposition rates of cobalt and nickel by reducing the power setting of their sources to $15 \mathrm{~W}$ and $85 \mathrm{~W} \mathrm{DC}$, respectively. We estimate that this will result in a film with a boron content of about $8 \%$. This is only slightly higher than that of the previously deposited films but the low deposition rate of boron, even at maximum power, prevents us from increasing the boron content any further. Figure 5.4 shows the ef- 


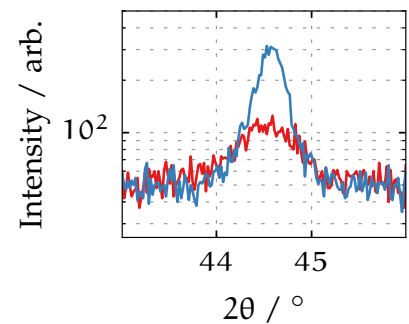

(a) $68 \mathrm{~nm}$ nickel.

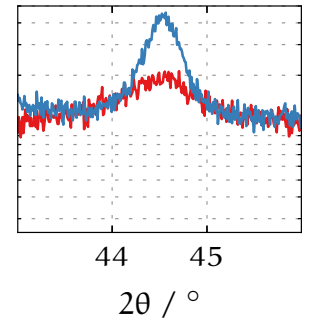

(b) $67 \mathrm{~nm} \mathrm{NiCo}$.

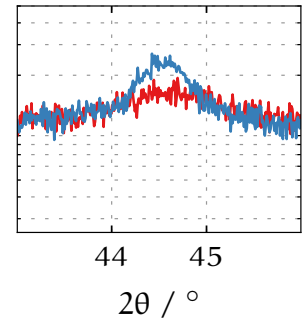

(c) $64 \mathrm{~nm} \mathrm{NiCoB}$.

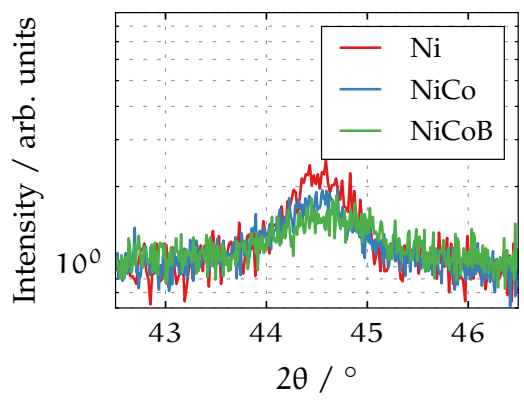

(d) As-deposited.

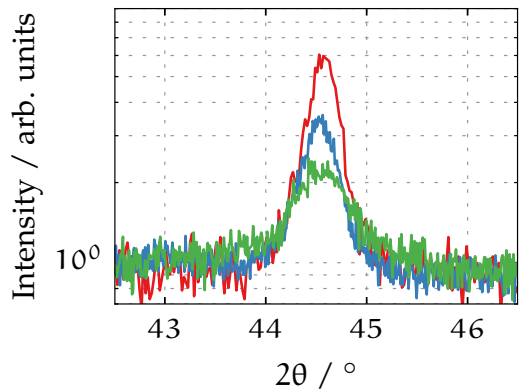

(e) Annealed at $500^{\circ} \mathrm{C}$ for $2.5 \mathrm{~h}$.

Figure 5.3: XRD recordings of the nickel fCC (III) peak for pure Ni, NiCo and $\mathrm{NiCoB}$ films on silicon with $4 \mathrm{~nm}$ boron capping layers as-deposited (red curves) and after annealing (blue curves) at $500{ }^{\circ} \mathrm{C}$ for $2 \mathrm{~h}$ in a nitrogen atmosphere. The pure nickel layer has the highest peak in both situations. The peak for asdeposited $\mathrm{NiCoB}$ is only marginally lower than that for $\mathrm{NiCo}$, but the increase in intensity after annealing is stronger for the NiCo layer. 


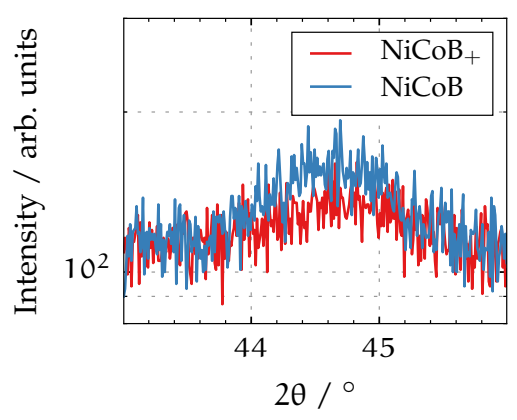

(a) As-deposited.

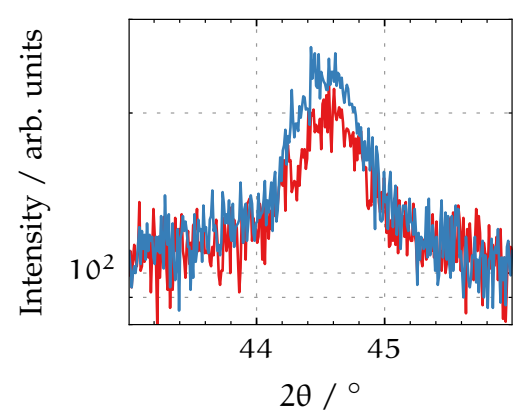

(b) Annealed at $500^{\circ} \mathrm{C}$ for $2.5 \mathrm{~h}$.

Figure 5.4: XRD comparison of the nickel fCC (111) peak of a NiCoB film on silicon with an increased boron content (about $8 \%$; red curve) with the NiCoB film shown in previous figure (blue curves), before and after annealing.

fects of the increased boron content on the crystallinity before and after annealing. The nickel (111) peak is somewhat lower for both cases showing a reduction in crystallinity.

In these measurements the boron content in the film influences the crystallinity both before and after annealing. For the growth of epitaxial films the crystallinity after annealing should be affected as little as possible by the additions needed to ensure deposition of an amorphous layer. The results shown so far were obtained from films deposited on a silicon (100) wafer with native oxide, that we do not expect to impose a preferential orientation for the deposited layer. A good match between the lattice of the substrate and the nickel (111) lattice is expected to increase the crystallinity of the film after annealing. Figure 5.5 shows XRD measurements of the nickel (111) peak of a NiCoB film on sapphire, lattice mismatch approximately $5 \%$. The much higher peaks, both before and after annealing, than for the alloy on silicon indicate a much higher crystallinity of the film. In addition to an increase in peak height, annealing also leads to broadening and a shift to smaller $\theta$ of the peak. The shift is likely caused by annealing induced relaxation of strain in the NiCo lattice due to the mismatch with the substrate. The broadening is attributed to defects in the film (Ungár, 2004). 


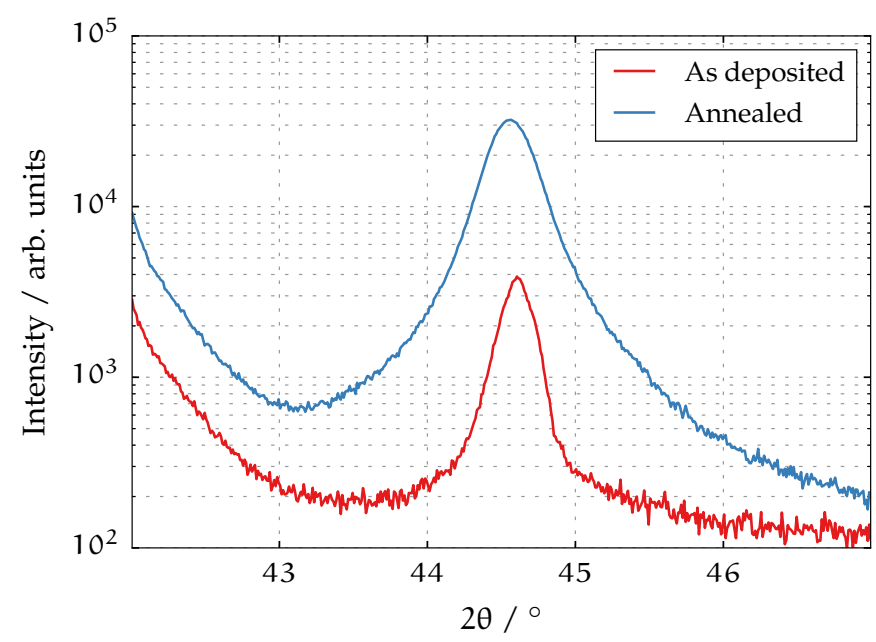

Figure 5.5: XRD measurements of a $60 \mathrm{~nm} \mathrm{NiCoB}$ film on a sapphire substrate before (red curve) and after $2.5 \mathrm{~h}$ annealing at $500{ }^{\circ} \mathrm{C}$. The as-deposited film already shows a strong nickel (111) peak. Annealing leads to an increase in the maximum intensity and a broadening of the peak. The onset of the sapphire (oo6) peak at $41.7^{\circ}$ can be seen at the left side of the plot.

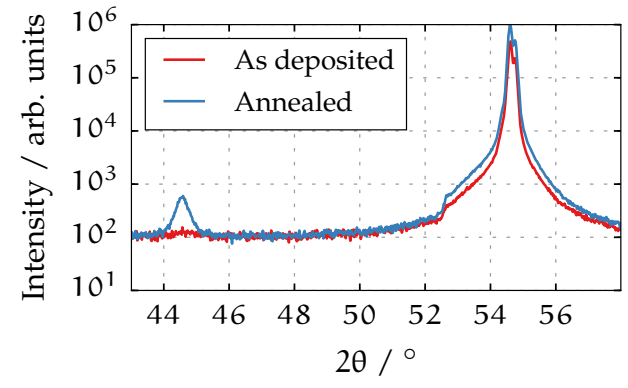

(a) Complete scan.

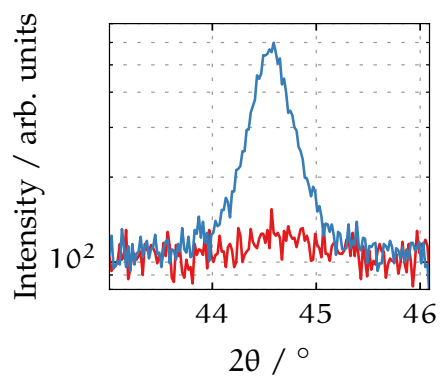

(b) Nickel fcc (111).

Figure 5.6: XRD curves of an NiCoB film on HOPG before and after annealing at $450^{\circ} \mathrm{C}$ for $3 \mathrm{~h}$. For the annealed $\mathrm{NiCoB}$ film (blue curve) a clear nickel (111) peak is visible at $44.5^{\circ}$ that is absent in the spectrum of the unannealed film, showing a large increase of crystallinity. An intense substrate peak is measured at $54.6^{\circ}$ (HOPG (o04)). 
The results so far are promising for the growth of an epitaxial NiCo layer on top of graphene. As long as the interaction with the graphene is sufficiently weak the right amount of boron in the alloy can suppress crystallisation. Upon annealing the good lattice match between graphene and the nickel (111) surface, mismatch approximately $2.5 \%$, should catalyse crystallisation of the alloy in the right orientation. The results of depositing and annealing a $64 \mathrm{~nm}$ NiCoB alloy on HOPG are shown in figure 5.6. The only peak in the XRD scan on the as-deposited sample is the HOPG (004) peak of the substrate, no signs of crystallisation in the alloy can be made out. The nickel (111) peak of the annealed sample has a peak-tobackground intensity ratio comparable to the annealed pure nickel film on silicon in figure $5 \cdot 3$.

Keeping in mind future devices using the shown technique to fabricate epitaxial top electrodes, some properties of the resulting films were investigated. Figure 5.7 shows AFM measurements of the effect of the annealing temperature on the film roughness. The sample annealed at $550^{\circ} \mathrm{C}$ shows a difference between the lowest and highest measured surface elevation approaching twice the deposited film thickness. At higher annealing temperature grains with favourable crystal orientations start forming to reduce the surface energy of the film. This grain formation can lead to the formation of isolated islands that are detrimental to the continuity of the film. All three annealed films show an increase in surface roughness compared to the as-deposited film. The XRD scans in figure 5.8 show no large differences in the crystallinity for the different annealing temperatures. To minimize the risk of forming a discontinuous film the annealing temperature should be chosen as low as possible while still causing strong crystallisation, in our case $450^{\circ} \mathrm{C}$.

Figure 5.9 shows the magnetic properties of two annealed $\mathrm{NiCoB}$ films on silicon. Both films show ferromagnetic behaviour with coercive fields of approximately $7 \mathrm{mT}$, high remanences, and saturation magnetic moments approaching $0.5 \mu \mathrm{A} \mathrm{m}^{2}$. These measurements were performed on square samples with sides of $5 \mathrm{~mm}$ and a $60 \mathrm{~nm}$ thick $\mathrm{NiCoB}$ film resulting in an estimated magnetisation of $333 \mathrm{kA} / \mathrm{m}$. Comparing with bulk saturation magnetisations for 


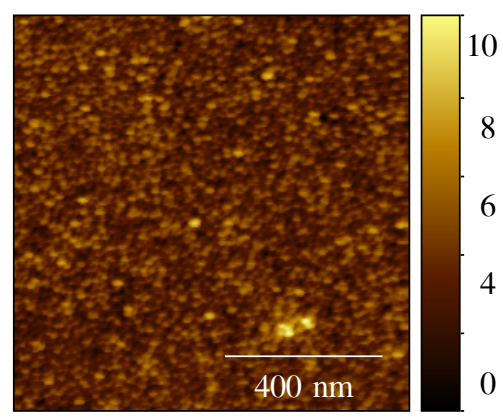

(a) As-deposited.

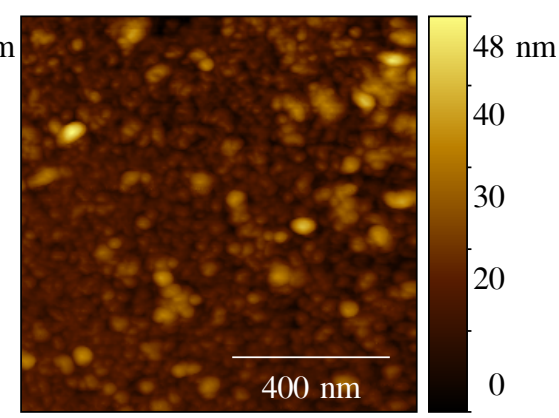

(b) $450^{\circ} \mathrm{C}$.

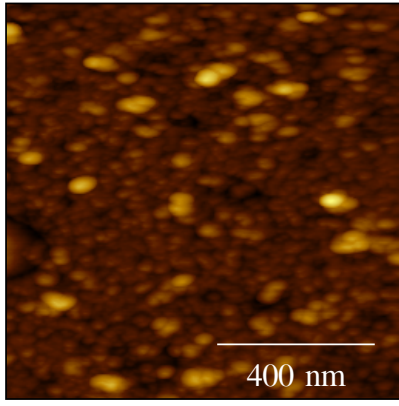

(c) $500{ }^{\circ} \mathrm{C}$.
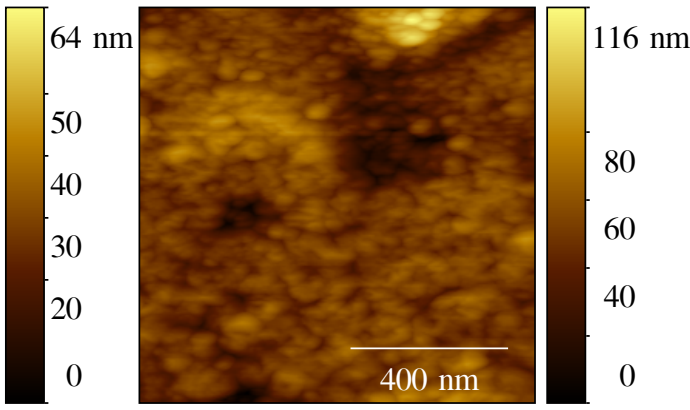

(d) $550^{\circ} \mathrm{C}$.

Figure 5.7: AFM scans of $60 \mathrm{~nm} \mathrm{NiCoB}$ on sapphire. A higher annealing temperature leads to a higher peak to valley height. 


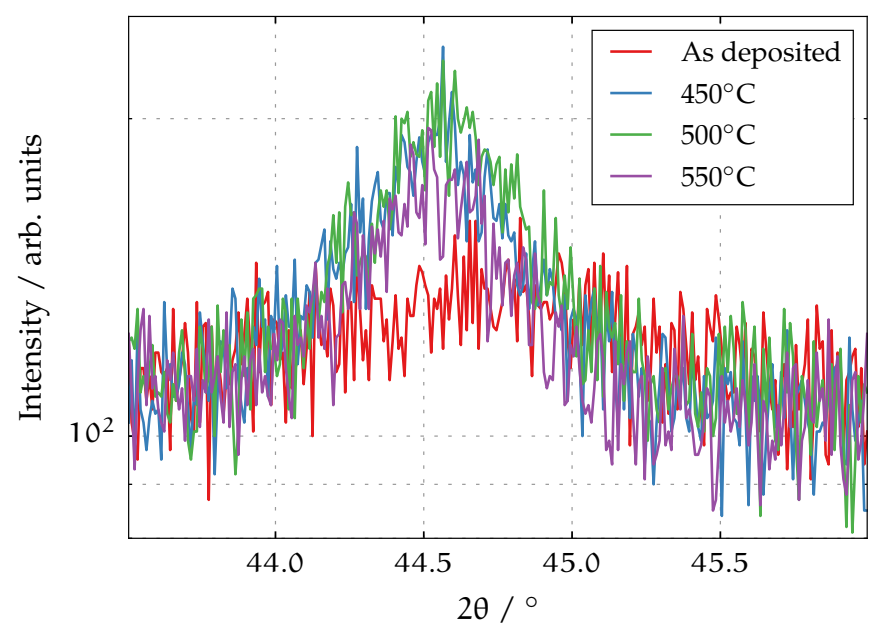

Figure 5.8: XRD measurements of the nickel (111) peak of $60 \mathrm{~nm} \mathrm{NiCoB}$ films on silicon annealed for $2.5 \mathrm{~h}$ at different temperatures. The peak heights and shapes after annealing are similar for all three annealing temperatures.

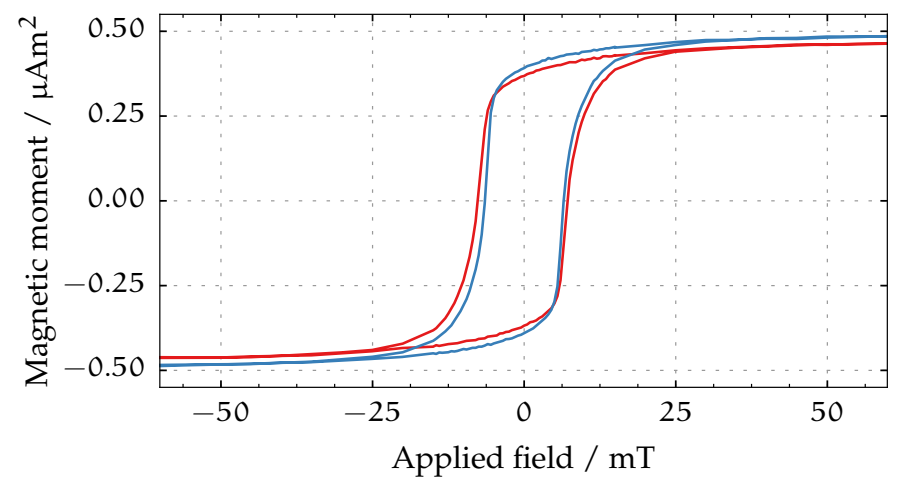

Figure 5.9: VSM measurements showing the effect of an externally applied magnetic field on the magnetisation of $60 \mathrm{~nm} \mathrm{NiCoB}$ on silicon, annealed for $2.5 \mathrm{~h}$ at $45 \mathrm{O}^{\circ} \mathrm{C}$. The external magnetic field is applied parallel to the sample surface and the magnetic moment is measured in the direction of the applied field. The red and blue curves belong to two separate samples. Both samples show ferromagnetic behaviour with comparable coercive fields and saturation magnetisations. 
nickel and cobalt of $510 \mathrm{kA} / \mathrm{m}$ and $1446 \mathrm{kA} / \mathrm{m}^{\dagger}$ we would expect a value closer to $630 \mathrm{kA} / \mathrm{m}$.

\subsection{CONCLUSION}

Promising results have been shown for the growth of epitaxial ferromagnetic top electrodes on graphene. When deposited on HOPG $\mathrm{NiCoB}$ forms an amorphous layer that can be crystallised by annealing at $450^{\circ} \mathrm{C}$. The annealed film shows a strong XRD peak matching a nickel fcc (111) preferential surface orientation. Clear ferromagnetic switching is observed in VSM measurements with a saturation magnetisation around half the value expected based on the composition of the film.

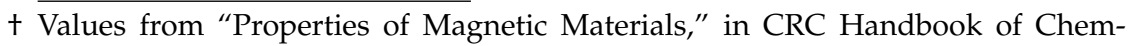
istry and Physics, 97th Edition (Internet Version 2017), W. M. Haynes, ed., CRC Press/Taylor \& Francis, Boca Raton, FL. 


\subsection{INTRODUCTION}

The field of organic spintronics offers the prospect of combining the advantageous properties of organic materials with those of spintronic phenomena. Spin based applications using inorganic materials have shown remarkable physical phenomena such as GMR (Baibich et al., 1988; Binasch et al., 1989), TMR (Jullière, 1975), spin Hall effect (D'yakonov and Perel, 1971; Hirsch, 1999; Kato et al., 2004), and spin transfer torque (Slonczewski, 1996). Several of these phenomena have found useful applications in everyday consumer electronics. It seems a logical step to combine the possibilities that spintronics has to offer with the flexibility, affordability, ease of manufacturing, and other advantages that the use of organic materials has brought to the field of electronics. The transport of charges and spins in organic semiconductors by hopping between the $\pi$-conjugated orbitals localised on different molecules (or polymer chain segments) is very different from the mechanism that applies for inorganic semiconductors, where charge carriers reside in fully delocalised bands. Theoretical models that agree with experimental results (Riminucci et al., 2013) and provide insight in the underlying principles are still being developed (Harmon and Flatté, 2014; Yu, 2013). While spin polarised transport in organic semiconductors has been studied for more than a decade (Xiong et al., 2004), conclusive proof that spin conserving transport is possible in organic materials has been elusive (Riminucci et al., 2013). Recently, spin pumping experiments have been reported that show spin transport in organics to be feasible (Watanabe et al., 2014). 
Not only the transport mechanism of spins or spin polarised charges is an important topic within organic spintronics, also the injection of spins from a ferromagnetic metal into an organic semiconductor is far from trivial. At the interface between the materials phenomena like orbital hybridisation and charge transfer can greatly influence the transport of spins across the interface. Spin polarised microscopy and spectroscopy techniques have shown these effects to be typically spin dependent, resulting in a hybrid interface with magnetic properties that are quite different from those of the bulk ferromagnet (Djeghloul et al., 2013; Lach et al., 2012; Rizzini et al., 2014; Sun et al., 2014; Suzuki et al., 2002, 2004; Tran et al., 2013). It is a topic of active study how this effects the spin transport across the interface (Brede et al., 2010; Kawahara et al., 2012; Raman et al., 2013; Schmaus et al., 2011; Steil et al., 2013). In addition, the magnetic properties, such as the coercive field of the saturation magnetisation, of ferromagnetic thin films may be tuned by the same interfacial effects (Bairagi et al., 2015; Moorsom et al., 2014). Very interestingly, it even turns out to be possible to induce ferromagnetism in thin diamagnetic copper layers by interface formation with $\mathrm{C}_{60}$ molecules (Al Ma'Mari et al., 2015). A growing body of density functional theory based studies are starting to provide insight into the physics at play at these interfaces and hint at the exciting possibility of actively tailoring the properties of hybrid systems (Atodiresei et al., 2010, 2011; Friedrich et al., 2015; Lazić et al., 2014).

In this study we investigate the changes in the magnetic properties of cobalt thin films when forming an interface with organic molecules. Since charge redistribution is expected to be a possible cause of these changes, we chose both a strong electron acceptor, TCNQ, and a strong electron donor, TTF, as the organic adsorbants. By investigating the effects of these complementary molecular species we shed light on the question whether systematic control of the interfacial magnetic properties through charge redistribution is feasible.

By recording X-ray absorption spectroscopy (XAS) data before and after deposition of the organic molecules we can study the changes in the electronic properties of a ferromagnetic cobalt thin film. The 
impact on the magnetic properties of these changes can be probed by X-ray magnetic circular dichroism (XMCD) using circularly polarised X-rays.

\subsection{MATERIALS AND METHODS}

All sample preparation steps and experiments were performed insitu at the I1011 beam line at the MAX-lab synchrotron radiation facility in Lund, Sweden.

Single crystal sapphire (0001) was used as a substrate because cobalt will readily grow as an epitaxial fcc (111) film when deposited onto the clean sapphire surface at room temperature (Wang et al., 2013; Yabuhara et al., 2011). The cobalt was evaporated onto the substrates under ultra-high vacuum conditions from an e-beam evaporator. The base pressure of the system varied between $9.0 \times 10^{-9}$ mbar and $1.5 \times 10^{-8}$ mbar during the experiments. We judged the thickness of the cobalt layers by the opacity of the film and estimated it to be between 10 and $20 \mathrm{~nm}$ for all samples.

The organic molecules (TCNQ and TTF) were deposited using a custom-made sublimation cell. The organic material (in powder form) was loaded into a small halogen light bulb, that we had cut open at the top, and sublimated by resistively heating the tungsten filament fitted inside the bulb. The sublimation cell was separated from the deposition chamber by a gate valve in order to prevent unwanted deposition of the organic material. For TTF, especially, this was essential as we found it to sublime fast enough for deposition at room temperature without any applied heating. To increase the sticking probability for TTF to the cobalt surface, the sample was cooled to liquid nitrogen temperature during TTF deposition. Deposition of TCNQ was carried out with the substrate held at room temperature.

In the used setup the samples could be heated by a resistive heater mounted on the sample stage, or cooled by a liquid nitrogen filled cold finger connected to the sample stage. A thermocouple 
connected to the sample stage gave an approximate reading of the sample temperature.

The X-rays for XAS and XMCD measurements were generated using an undulator that is inserted into the synchrotron storage ring. The elliptically polarising undulator allowed for the use of X-rays with a degree of circularity of about $90 \%$. The sample was mounted at an angle of 60 degree between the sample normal and the incoming photon beam. To correct for these experimental parameters the magnetic moments extracted from the XMCD spectra were multiplied by $1 /\left(0.9 \times \sin 60^{\circ}\right) \approx 1.28$. During measurements the sample sat between a pair of water-cooled copper coils that could generate magnetic fields up to $65 \mathrm{mT}$. The sample could be transferred back and forth between deposition and measurement chamber without breaking ultra-high vacuum conditions.

X-ray absorption spectra were recorded in the total electron yield (TEY) mode by measuring the drain current from the sample to ground while varying the incident photon energy. To ensure good electrical contact between the deposited cobalt films and the sample holder we deposited a strip of tungsten along one edge of our sapphire substrates by magnetron sputtering prior to the experiments. By taking the difference of a pair of XAS spectra recorded with opposite relative orientations of the sample magnetisation and light polarisation, an XMCD spectrum was obtained, providing insight into the orbital and spin magnetic moments of the sample.

To be able to quantitatively compare the different recorded XAS spectra, these were corrected for changes in the intensity of the incident photon beam. To measure this intensity a gold grid was placed in the path of the photon beam before it hit the sample. The drain current from this grid is proportional to the intensity of the photon beam. By taking the ratio of the sample drain current over the gold-grid drain current, $\mathrm{I}_{0}$, the spectra are normalized for changes in the incoming beam.

Magnetisation loops could be recorded by tuning the photon energy to the maximum of the $L_{3}$ peak of the cobalt $L$-edge and varying the external magnetic field. This method provides magneti- 


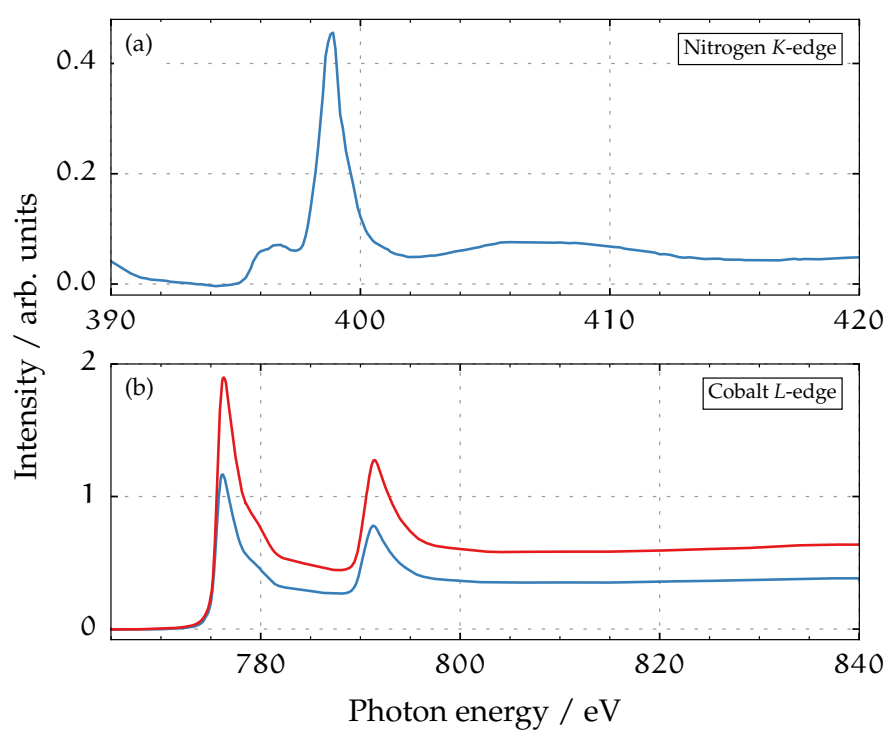

Figure 6.1: Nitrogen $K$-edge (a) and cobalt $L$-edge (b) for the clean cobalt thin film (red curve), and after deposition of a layer of TCNQ on top of the cobalt (blue curves). The spectra are normalized using reference spectra recorded with the gold grid and the pre-edge intensity is subtracted. Both the presence of the nitrogen $K$-edge peak and the attenuation of the cobalt $L$-edge peak demonstrate the formation of a TCNQ film. Comparison of the recorded nitrogen $K$-edge with that of the neutral TCNQ molecule (Fraxedas et al., 2003) shows clear differences indicative of chemisorption.

sation values relative to the absorption peak height, so no actual magnetisation values.

\subsection{RESULTS AND DISCUSSION}

TCNQ on cobalt

For the first set of measurements a film of TCNQ is deposited on a fcc cobalt film. The sample is at room temperature during all 


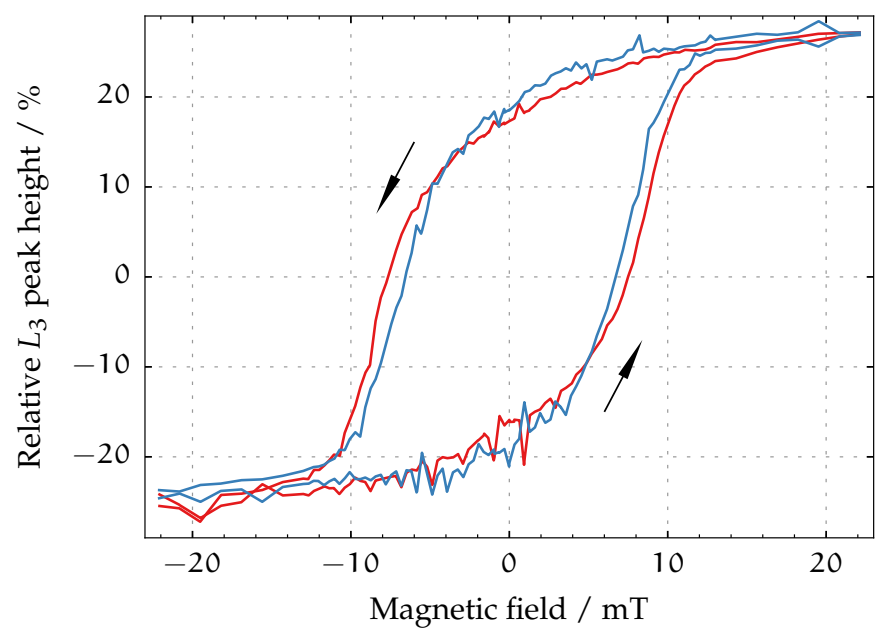

Figure 6.2: Magnetic field sweep of the intensity of the cobalt $L_{3}$ peak for the clean cobalt thin film (red curve), and after deposition of a layer of TCNQ on top of the cobalt (blue curve). The latter exhibits a slightly lower coercivity and higher squareness. The arrows indicate the sweep direction of the magnetic field. 

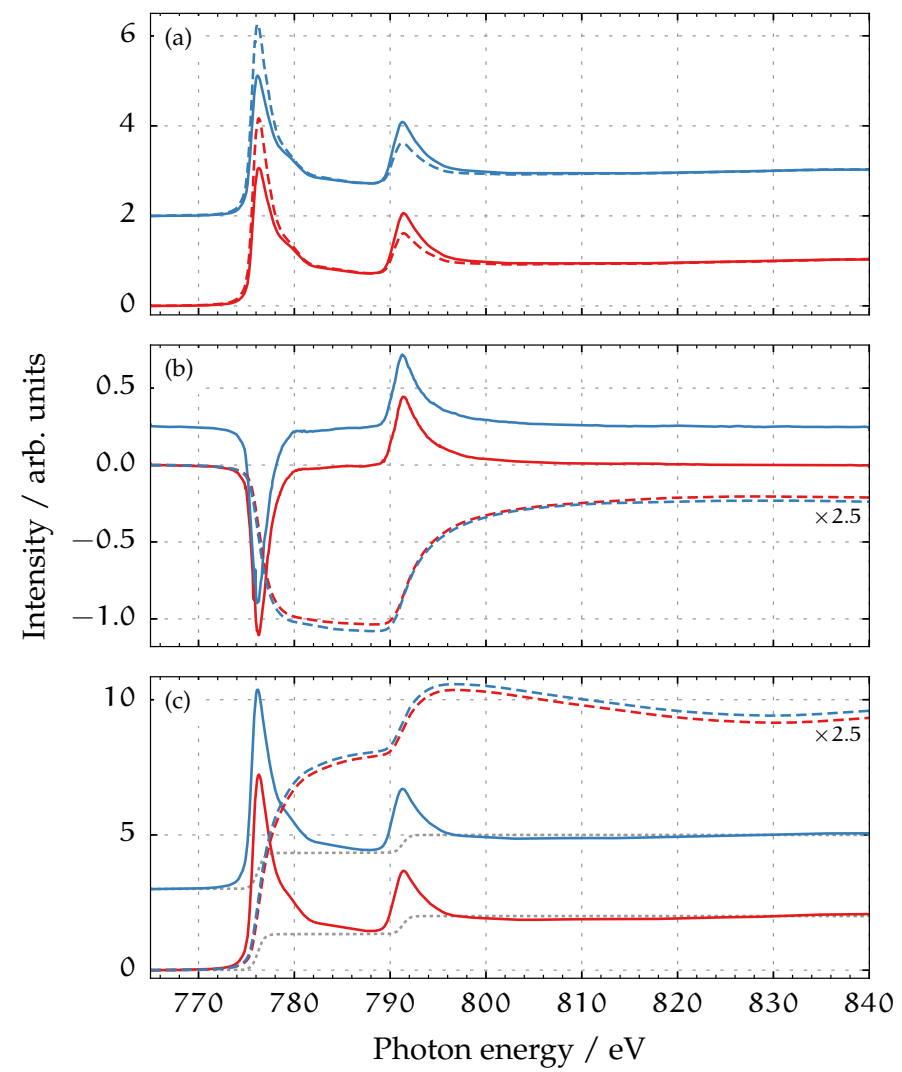

Figure 6.3: cobalt L-edge XAS and XMCD spectra before (red curves) and after (blue curves; vertical offset for clarity) deposition of TCNQ. (a) Measured XAS spectra for opposite relative orientations of sample magnetisation and photon polarisation (solid and dashed lines). To do proper XMCD analysis the pre-edge intensity is subtracted from the spectra and the intensity at high photon energy far beyond the edge, where magnetic effects are absent, is normalized to unity. (b) XMCD spectra, difference between XAS spectra in (a) (solid), and integrated XMCD spectra (dashed; scaled down by a factor of 2.5 for clarity). (c) Summed XAS spectra (solid), and integrated sum spectra (dashed; scaled down by a factor of 2.5 for clarity) after subtraction of two-step-like edge-jump background (dotted, grey line). The variation in the integrated intensity at high photon energy is an artefact of the background subtraction procedure, which is hampered by the structure in the background. 
depositions and measurements. The deposition of the TCNQ layer is confirmed by the appearance of a strong nitrogen $1 s$ (K-edge) absorption peak (figure 6.1a). The features in the nitrogen $K$-edge XAS spectrum of TCNQ adsorbed on cobalt are distinctly different from those of the neutral molecule (Fraxedas et al., 2003), which is indicative of chemisorption and thus hybridisation of electronic states. The $\mathrm{N}(1 S) \rightarrow \pi^{*}$ peak at the onset of the $K$-edge near $395 \mathrm{eV}$ clearly exhibits two split components. The corresponding feature of the neutral molecule is a single asymmetric peak, and has been attributed to excitations into the nearly degenerate $a_{u}$ and $b_{1 u} \pi^{*}$ orbitals (where the symmetry labels refer to $D_{2 h}$ symmetry) (Fraxedas et al., 2003). For the neutral molecule, a small peak associated with the $b_{2 g} \pi^{*}$ orbital is present on the high excitation energy side of the main $\mathrm{N}(1 s) \pi \rightarrow *$ resonance, which is absent for TCNQ on cobalt. Since TCNQ is a strong electron acceptor, it is expected that electrons have been transferred from cobalt to TCNQ at the interface.

The presence of the TCNQ layer also leads to the attenuation of the cobalt $2 p$ ( $L$-edge) absorption spectrum (figure 6.Ib). The amount of attenuation can be used to give an estimate of the thickness of the deposited inorganic layer. Based on measurements of the attenuation of TEY XAS peaks by overlayers (Frazer et al., 2003; Vogel and Sacchi, 1994) we estimate the thickness of the organic film to be around $0.5 \mathrm{~nm}$.

Due to the spin orbit coupling of the cobalt $2 p$ electronic states, the transition probabilities at the $L_{2,3}$-edge depend on the relative orientations of the magnetisation of the sample and the helicity vector of the incoming light. By sweeping the magnetisation while keeping the photon polarisation fixed and at an energy matching the maximum of the $L_{3}$-edge of cobalt, the magnetic hysteresis curve of figure 6.2 is measured. The deposition of TCNQ slightly lowers the coercivity of the cobalt and increases the squareness (remanence at zero applied field relative to saturation magnetisation).

The XMCD spectra of the cobalt L-edge (figure 6.3) show negligible change after TCNQ deposition. Sum rule analysis of the spectra (Chen et al., 1995) gives a ratio of the orbital contribution to the spin contribution for the angular momentum of 0.051 for the clean 
cobalt thin film and 0.056 for the film with TCNQ. Due to the structure in the background after the absorption edge it is difficult to determine quantitative values for the respective spin and orbital angular momenta. Our data suggest a value between 2.0 $\mu_{\mathrm{B}}$ and $2.3 \mu_{\mathrm{B}}$ per atom for the spin angular momentum, and between $0.10 \mu_{B}$ and $0.13 \mu_{\mathrm{B}}$ per atom for the orbital angular momentum. An overview of all calculated magnetic moments is given in table 6.1. Note that the different values for $\mathrm{m}_{\text {orb }} / \mathrm{m}_{\text {spin }}$ of the two clean cobalt films in table 6.1 might be related to a difference in film thickness in the $10 \mathrm{~nm}$ to $20 \mathrm{~nm}$ range, such that a different surface-to-bulk ratio of the magnetic moments is observed in the TEY-mode XAS measurements.

\section{TTF on cobalt}

After replacing the TCNQ sublimation cell with one filled with TTF, the readings of the mass spectrometer for residual gas analysis, mounted on the preparation chamber, confirmed that the TTF sublimation rate was sufficiently high for TTF deposition while keeping the source material at room temperature. The absence of any carbon $\mathrm{K}$-edge signal in the XAS spectrum (figure 6.4) after exposing the cobalt surface, held at room temperature, to TTF vapour for a few minutes indicates a low sticking probability of the molecules to the surface. To achieve TTF deposition the sample is cooled, using liquid nitrogen, to approximately $100 \mathrm{~K}$ during exposure to the TTF vapour. The appearance of a clear carbon $K$-edge signal affirms that a layer of TTF is indeed deposited on the surface under these conditions.

The carbon K-edge absorption shows a double main peak, whereas the neutral molecule shows a single peak (Fraxedas et al., 2003). This splitting is attributed to hybridisation of the cobalt surface with the different molecular orbitals that contribute to this peak.

For the most prominent contribution of interface effects to the measured spectra a TTF film thickness of a single monolayer would be ideal. Charge transfer between the cobalt and the TTF molecules at the interface is expected to lead to significant binding of these 


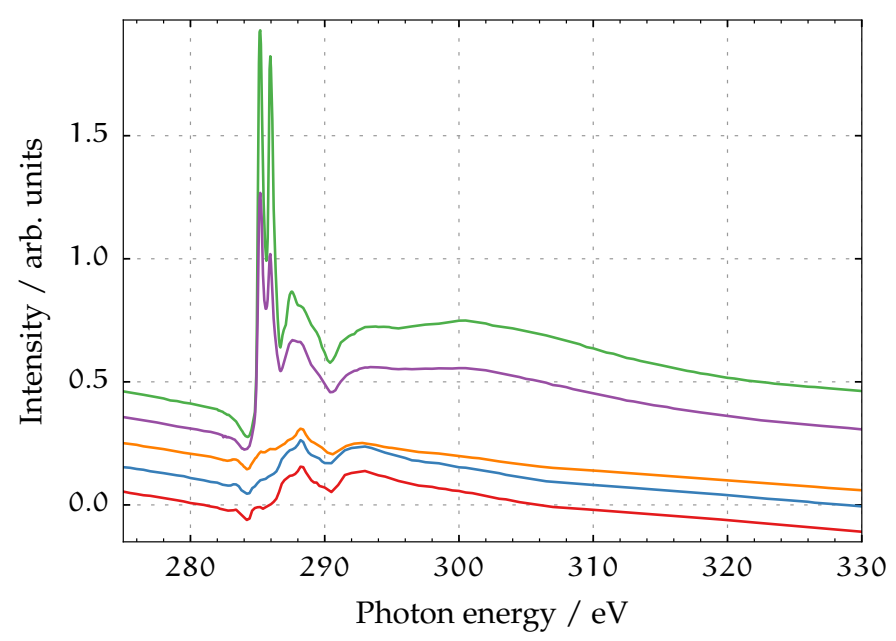

Figure 6.4: XAS spectrum of the carbon $K$-edge of TTF covered cobalt (vertical offset for clarity). The red and blue curves for the clean cobalt surface, respectively, before and after exposure to TTF vapour at room temperature are very similar, indicating no noteworthy deposition of TTF. The structure at the carbon $K$-edge in these spectra results from adventitious carbon on the beam line optics, which is only partially corrected for by the $\mathrm{I}_{0}$ measurement. Clear peaks are observed after deposition with the sample cooled down to about $100 \mathrm{~K}$ (green curve). After heating to $283 \mathrm{~K}$ the peaks are attenuated (magenta curve), indicating partial desorption of TTF. After heating of the sample to $323 \mathrm{~K}$ for about $5 \mathrm{~min}$ (orange curve) the spectrum resembles that of the clean cobalt thin film, suggesting that the TTF layer is completely removed. 


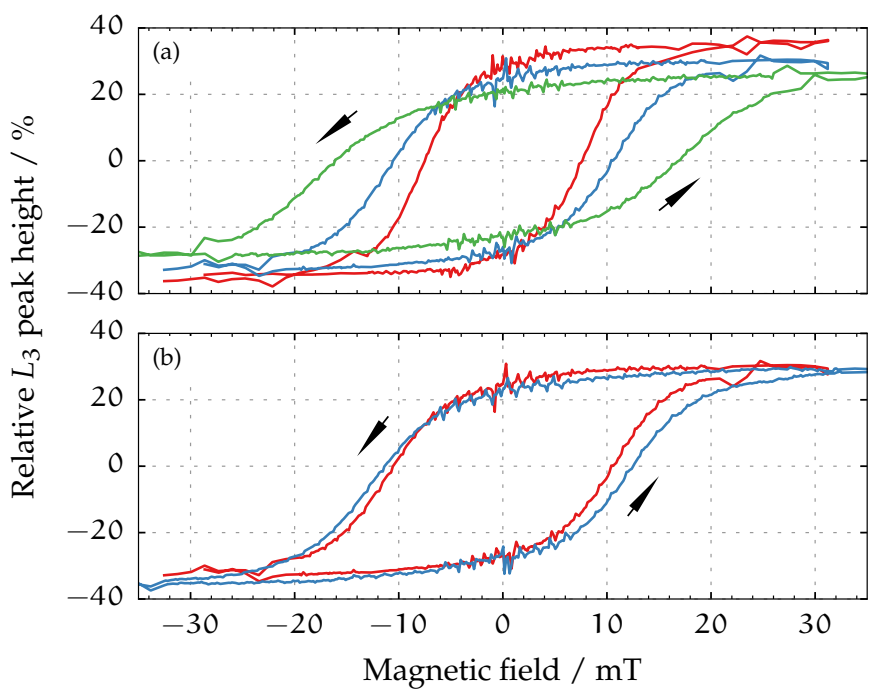

Figure 6.5: Magnetic hystersis the of cobalt $L_{3}$ XAS peak height. (a) Hysteresis for the clean cobalt thin film measured at room temperature (red curve), at $100 \mathrm{~K}$ (blue curve), and after deposition of a layer of TTF at 1oo K (green curve). The TTF layer clearly increases the coercivity and reduces the squareness of the cobalt film. (b) Hysteresis curve measured at $100 \mathrm{~K}$ before TTF deposition (red curve) and after TTF deposition and subsequent heating to $323 \mathrm{~K}$ for $5 \mathrm{~min}$ (blue curve). After removal of the TTF layer the magentic hysteresis of the cobalt film reverts to its original shape. 

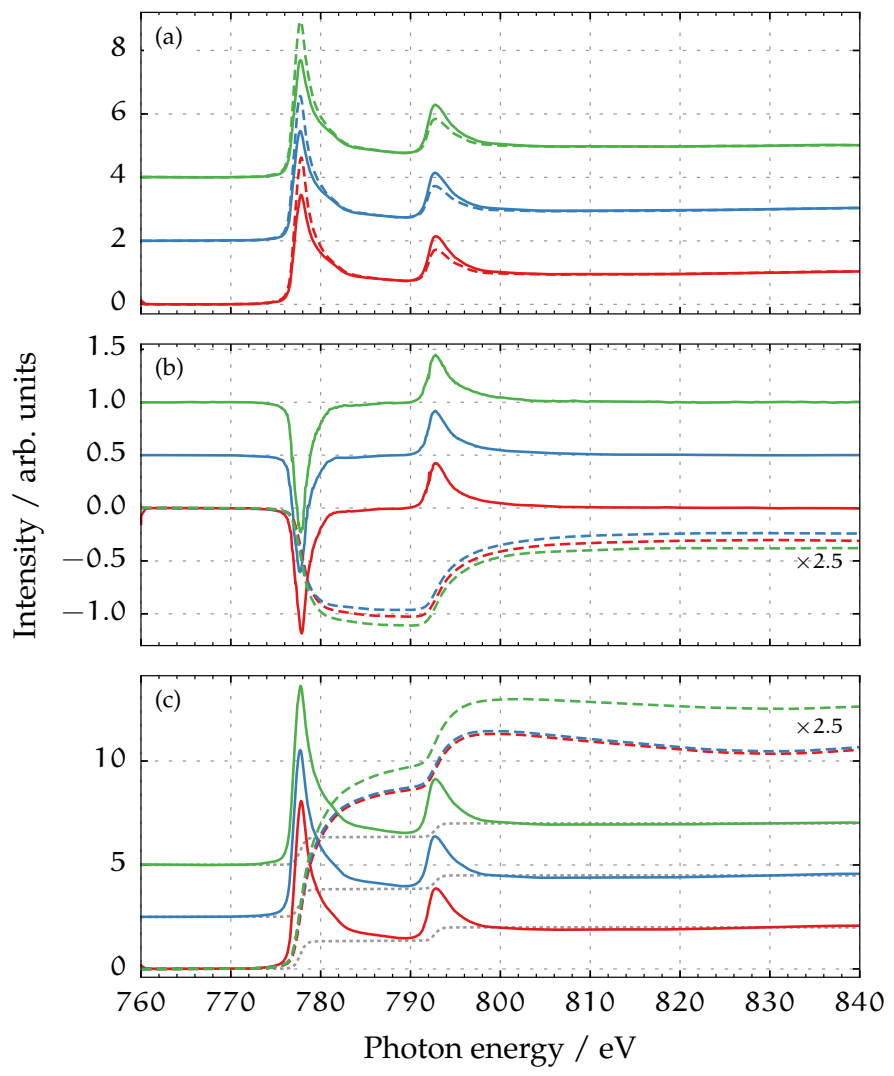

Figure 6.6: cobalt L-edge XAS and XMCD spectra of the clean cobalt film measured at room temperature (red curves) and at $100 \mathrm{~K}$ (blue curves; vertical offset for clarity), and after deposition of TTF measured at $100 \mathrm{~K}$ (green curves; vertical offset for clarity). (a) Measured XAS spectra for opposite relative orientations of sample magnetisation and photon polarisation (solid and dashed lines). To do proper XMCD analysis the pre-edge intensity is subtracted from the spectra and the intensity at high photon energy far beyond the edge, where magnetic effects are absent, is normalized to unity. (b) XMCD spectra, difference between XAS spectra in (a) (solid), and integrated XMCD spectra (dashed; scaled down by a factor of 2.5 for clarity). (c) Summed XAS spectra (solid), and integrated sum spectra (dashed; scaled down by a factor of 2.5 for clarity) after subtraction of two-step-like edge-jump background (dotted, grey line). The variation in the integrated intensity at the high photon energy is an artefact of the background subtraction procedure, which is hampered by the structure in the background. 
molecules. By gradually heating the sample we aimed to remove all TTF except for the more strongly bound first monolayer. After heating the sample to $283 \mathrm{~K}$ for several minutes the reduced film thickness leads to attenuation of the $C$ peak. After subsequent heating to $323 \mathrm{~K}$ the TTF seems to be completely removed from the sample.

The magnetic hysteresis of the cobalt is strongly influenced by the deposition of a layer of TTF (figure 6.5). The coercivity is increased by a factor of 1.5 and the magnetisation saturates more gradually. After heating to $323 \mathrm{~K}$ the magnetic behaviour reverts to that of the clean cobalt film, showing once more that the TTF layer is removed.

The XMCD analysis of the absorption spectra (figure 6.6) shows strong variations in the ratio of the orbital to the spin magnetic moment. For the clean cobalt film at room temperature the ratio is 0.081 ; at $100 \mathrm{~K}, 0.065$; and after TTF deposition at $100 \mathrm{~K}, 0.099$. For the actual magnetic moments the data give a value between $0.14 \mu_{\mathrm{B}}$ and $0.15 \mu_{\mathrm{B}}$ per atom for the orbital contribution of the clean film at room temperature and between $1.7 \mu_{\mathrm{B}}$ and $1.8 \mu_{\mathrm{B}}$ per atom for the spin contribution. Measured at $100 \mathrm{~K}$ both moments decrease to between $0.11 \mu_{\mathrm{B}}$ and $0.12 \mu_{\mathrm{B}}$ per atom for the orbital part and between $1.6 \mu_{\mathrm{B}}$ and $1.8 \mu_{\mathrm{B}}$ per atom for the spin. After deposition of TTF the orbital moment increases again to between $0.15 \mu_{\mathrm{B}}$ and $0.16 \mu_{\mathrm{B}}$ per atom and the spin moment decreases to between $1.5 \mu_{\mathrm{B}}$ and $1.6 \mu_{\mathrm{B}}$ per atom (see table 6.1 for an overview). It should again be noted that these values are expected to have substantial errors due to the non-uniform background at the high energy side of the absorption edge. These errors are systematic for all measurements, therefore the values can be used for qualitative comparisons between the different spectra.

The effects of TTF deposition on the magnetisation reversal of the cobalt films are strong, while the changes in the XMCD spectra are subtle. The latter can in part be attributed to the finite probing depth of the TEY technique, meaning that only a small fraction of the cobalt $L$-edge signal stems from the outermost cobalt atoms that are primarily affected by bonding to TTF molecules. In addition, the effects of adsorption of molecules on ferromagnetic surfaces 
Table 6.1: Overview of magnetic moments calculated by sum rule analysis (Chen et al., 1995) of the measured XMCD spectra. All values are in units of $\mu_{\mathrm{B}}$ /atom. Our data show an increase of the ratio of the orbital magnetic moment to the spin magnetic moment after depostion of both TCNQ and TTF on a clean cobalt thin film.

\begin{tabular}{lccc}
\hline \hline & $\mathrm{m}_{\mathrm{orb}} / \mathrm{m}_{\text {spin }}$ & $\mathrm{m}_{\mathrm{orb}}$ & $\mathrm{m}_{\text {spin }}$ \\
\cline { 2 - 4 } TCNQ on cobalt & & & \\
\hline Clean cobalt film & 0.051 & $0.10-0.12$ & $2.0-2.3$ \\
TCNQ on cobalt & 0.056 & $0.11-0.13$ & $2.0-2.3$
\end{tabular}

\section{TTF on cobalt}

\begin{tabular}{llll}
\hline Clean cobalt film (293 K) & 0.081 & $0.14-0.15$ & $1.7-1.8$ \\
Clean cobalt film (100 K) & 0.065 & $0.11-0.12$ & $1.6-1.8$ \\
TTF on cobalt (100 K) & 0.099 & $0.15-0.16$ & $1.5-1.6$ \\
\hline \hline
\end{tabular}

are strongly inhomogeneous (Brede et al., 2010; Tran et al., 2013), and an area averaged XMCD measurement is not representative of any significant, local modifications of surface magnetic moments. On the other hand, the magnetic anisotropy energy, which has a significant effect on the magnetic hysteresis loops, may be strongly affected by such local modifications (Bairagi et al., 2015).

\subsection{CONCLUSION}

In summary, the XAS spectra for both TCNQ and TTF molecular layers on top of a ferromagnetic cobalt thin film have been measured. Hybridisation of molecular orbitals with the cobalt interface can be inferred from clear differences between the recorded spectra and those of the respective neutral molecules.

XMCD analysis of the samples, using different relative orientations of sample magnetisation and light polarisation, suggests that 
both TCNQ and TTF lead to an increase of the ratio of the orbital magnetic moment to the spin magnetic moment.

Magnetic field sweeps of the cobalt $L_{3}$ peak intensity show that the coercivity of the cobalt film decreases slightly when the molecular electron acceptor TCNQ is deposited on the surface, and increases with a factor of $\sim 1.5$ when the donor TTF is deposited on top. This complementary behaviour shows the possibility of controlled tuning of the properties of the cobalt interface by deposition of the suitable organic semiconductor. 



\section{CONCLUSION AND OUTLOOK}

We have shown advances in and novel methods for the research field of organic spintronics. We developed a fabrication method to create devices containing a nickel-graphene interface, that allows for the flexible selection of the specific area of a nickel-graphene substrate that is contacted. By directly using the wafer on which the graphene was grown we maximize the changes of an epitaxial relation between the nickel and the graphene, and we minimize the risk of contaminating the interface. The freedom of choice for the top electrode in this method allows for a large range of possible transport experiments.

A possible improvement of the fabrication method would be the use of atomic layer deposition (ALD) for the deposition of the insulating layers. Successful growth of high-quality oxides on graphene using ALD has been reported and would allow for much thinner oxide layers. This would facilitate the realization of devices with thin top electrodes, e.g., for Tedrow-Meservey style measurement of the spin polarisation.

One of the big hurdles in this research is the graphene itself. The very small interaction of graphene with its environment negatively impacts the adhesion of any overlayer and complicates the formation of an epitaxial interface.

Transport measurements that were performed on successfully created devices show values for the out-of-plane TAMR ratio up to $50 \%$, much higher than the values reported for other systems with strong SOC. These high values are attributed to a combination of the expected strong Rashba SOC at the nickel-graphene interface and the $k$-vector filtering effect that favours the transport of electrons for which this effect is strongest. 
Promising results have been achieved using a method for the growth of an epitaxial ferromagnetic layer on top of graphene by annealing of an amorphous alloy of nickel, cobalt and boron. Combining this method of creating top electrodes with our fabrication method on nickel-graphene layers opens up to possibility to create a nickel-graphene-nickel stack with epitaxial interfaces on both sides of the graphene. Based on the predicted spin filtering at this specific interface, very high MR ratios should be attainable in such a device.

The experiments on molecular layers of TTF and TCNQ on top of cobalt thin films show the importance of the interface for spintronic applications. The observed complementary influence on the interfacial magnetic properties show that control over the quality of and thorough understanding of the physics of hybrid interfaces are the key to tailoring device behaviour within the field of organic spintronics.

Some years ago graphene was heralded as the material of the future, a huge evolutionary step in electronics. I am convinced that graphene will still be around in laboratories for quite some time, although other $2 \mathrm{D}$ materials are rapidly gaining interest possibly at the expense of graphene. I do fear, however, that the lack of robust methods for incorporating graphene into more complicated device structures will limit its technological applicability to that of a transparent and flexible conductor or as a reinforcement agent in composite materials. Quite a disappointment considering the broad array of its (predicted) extraordinary properties.

I am more optimistic for the fields of organic spintronics as a whole, both scientifically and technologically. There is still much to learn about organic and hybrid electronics and the ongoing discovery of novel physical phenomena keeps the research moving forward. And the future will bring forth applications that we haven't even begun to imagine. 
Al Ma'Mari, F., Moorsom, T., Teobaldi, G., Deacon, W., Prokscha, T., Luetkens, H., Lee, S., Sterbinsky, G. E., Arena, D. A., MacLaren, D. A., Flokstra, M., Ali, M., Wheeler, M. C., Burnell, G., Hickey, B. J., and Cespedes, O. (2015). „Beating the Stoner criterion using molecular interfaces". Nature 524 (7563), 69-73.

Atodiresei, N., Brede, J., Lazić, P., Caciuc, V., Hoffmann, G., Wiesendanger, R., and Blügel, S. (2010). „Design of the local spin polarization at the organic-ferromagnetic interface". Physical Review Letters 105 (6), 066601.

Atodiresei, N., Caciuc, V., Lazić, P., and Blügel, S. (2011). „Engineering the magnetic properties of hybrid organic-ferromagnetic interfaces by molecular chemical functionalization". Physical Review B 84 (17), 172402.

Baibich, M. N., Broto, J. M., Fert, A., Van Dau, F. N., Petroff, F., Eitenne, P., Creuzet, G., Friederich, A., and Chazelas, J. (1988). "Giant magnetoresistance of (oo1)Fe/(oo1)Cr magnetic superlattices". Physical Review Letters 61 (21), 2472-2475.

Bairagi, K., Bellec, A., Repain, V., Chacon, C., Girard, Y., Garreau, Y., Lagoute, J., Rousset, S., Breitwieser, R., Hu, Y.-C., Chao, Y. C., Pai, W. W., Li, D., Smogunov, A., and Barreteau, C. (2015). "Tuning the magnetic anisotropy at a molecule-metal interface". Physical Review Letters 114 (24), 247203.

Barraud, C., Seneor, P., Mattana, R., Fusil, S., Bouzehouane, K., Deranlot, C., Graziosi, P., Hueso, L., Bergenti, I., Dediu, V., Petroff, F., and Fert, A. (2010). "Unravelling the role of the interface for spin injection into organic semiconductors". Nature Physics $6(8), 615-620$. 
Berger, L. (1964). „Influence of spin-orbit interaction on the transport processes in ferromagnetic nickel alloys, in the presence of a degeneracy of the $3 \mathrm{~d}$ band". Physica 30 (6), 1141-1159.

Binasch, G., Grünberg, P., Saurenbach, F., and Zinn, W. (1989). „Enhanced magnetoresistance in layered magnetic structures with antiferromagnetic interlayer exchange". Physical Review $B$ 39 (7), 4828-4830.

Bragg, W. H. and Bragg, W. L. (1913). „The reflection of X-rays by crystals". Proceedings of the Royal Society of London. Series A, Containing Papers of a Mathematical and Physical Character 88 (605), 428-438.

Brede, J., Atodiresei, N., Kuck, S., Lazić, P., Caciuc, V., Morikawa, Y., Hoffmann, G., Blügel, S., and Wiesendanger, R. (2010). „Spinand energy-dependent tunneling through a single molecule with intramolecular spatial resolution". Physical Review Letters 105 (4), 047204.

Butler, W., Zhang, X.-G., Schulthess, T., and MacLaren, J. (2001). "Spin-dependent tunneling conductance of $\mathrm{Fe}|\mathrm{MgO}| \mathrm{Fe}$ sandwiches". Physical Review B 63 (5), 054416.

Bychkov, Y. A. and Rashba, E. I. (1984). „Properties of a 2D electron gas with lifted spectral degeneracy". JETP lett 39 (2), 78.

Campbell, I. A., Fert, A., and Pomeroy, A. R. (1967). „Evidence for two current conduction iron". Philosophical Magazine 15 (137), 977983.

Castro Neto, A. H., Guinea, F., Peres, N. M. R., Novoselov, K. S., and Geim, A. K. (2009). ",The electronic properties of graphene”. Reviews of modern physics 81 (1), 109.

Chantis, A. N., Belashchenko, K. D., Tsymbal, E. Y., and van Schilfgaarde, M. (2007). „Tunneling anisotropic magnetoresistance driven by resonant surface states: first-principles calculations on an Fe(0o1) surface". Physical review letters 98 (4), 046601.

Chen, C. T., Idzerda, Y., Lin, H. J., Smith, N. V., Meigs, G., Chaban, E., Ho, G. H., Pellegrin, E., and Sette, F. (1995). „Experimen- 
tal confirmation of the X-ray magnetic circular dichroism sum rules for iron and cobalt". Physical Review Letters 75 (1), 152155.

Cobas, E., Friedman, A. L., van't Erve, O. M., Robinson, J. T., and Jonker, B. T. (2012). "Graphene as a tunnel barrier: graphenebased magnetic tunnel junctions". Nano letters 12 (6), 30003004 .

Dediu, V. A., Hueso, L. E., Bergenti, I., and Taliani, C. (2009). „Spin routes in organic semiconductors". Nature Materials 8 (9), 707716.

Dediu, V., Murgia, M., Matacotta, F. C., Taliani, C., and Barbanera, S. (2002). „Room temperature spin polarized injection in organic semiconductor". Solid State Communications 122 (3), 181-184.

Dedkov, Y. S., Fonin, M., Rüdiger, U., and Laubschat, C. (2008). "Rashba effect in the graphene/Ni(111) system". Physical review letters 100 (10), 107602.

Djayaprawira, D. D., Tsunekawa, K., Nagai, M., Maehara, H., Yamagata, S., Watanabe, N., Yuasa, S., Suzuki, Y., and Ando, K. (2005). „230\% room-temperature magnetoresistance in $\mathrm{CoFeB} /$ $\mathrm{MgO} / \mathrm{CoFeB}$ magnetic tunnel junctions". Applied Physics Letters 86 (9), 092502.

Djeghloul, F., Ibrahim, F., Cantoni, M., Bowen, M., Joly, L., Boukari, S., Ohresser, P., Bertran, F., Le Févre, P., Thakur, P., Scheurer, F., Miyamachi, T., Mattana, R., Seneor, P., Jaafar, A., Rinaldi, C., Javaid, S., Arabski, J., Kappler, J.-P., Wulfhekel, W., Brookes, N. B., Bertacco, R., Taleb-Ibrahimi, A., Alouani, M., Beaurepaire, E., and Weber, W. (2013). „Direct observation of a highly spinpolarized organic spinterface at room temperature". Scientific Reports 3, 1272.

D'yakonov, M. I. and Perel, V. I. (1971). „Possibility of orienting electron spins with current". JETP Letters-USSR 13 (11), 467469.

Ferrari, A. C., Meyer, J. C., Scardaci, V., Casiraghi, C., Lazzeri, M., Mauri, F., Piscanec, S., Jiang, D., Novoselov, K. S., Roth, S., 
and Geim, A. K. (2006). „Raman spectrum of graphene and graphene layers". Physical review letters 97 (18), 187401.

Fert, A. and Campbell, I. A. (1968). "Two-current conduction in nickel". Physical Review Letters 21 (16), 1190.

Fraxedas, J., Lee, Y. J., Jiménez, I., Gago, R., Nieminen, R. M., Ordejon, P., and Canadell, E. (2003). "Characterization of the unoccupied and partially occupied states of TTF-TCNQ by XANES and first-principles calculations". Physical Review B 68 (19), 195115.

Frazer, B. H., Gilbert, B., Sonderegger, B. R., and De Stasio, G. (2003). „The probing depth of total electron yield in the sub-keV range: TEY-XAS and X-PEEM". Surface Science 537 (1-3), 161-167.

Friedrich, R., Caciuc, V., Kiselev, N. S., Atodiresei, N., and Blügel, S. (2015). "Chemically functionalized magnetic exchange interactions of hybrid organic-ferromagnetic metal interfaces". Physical Review B 91 (11), 115432.

Gao, L., Jiang, X., Yang, S.-H., Burton, J. D., Tsymbal, E. Y., and Parkin, S. S. P. (2007). „Bias voltage dependence of tunneling anisotropic magnetoresistance in magnetic tunnel junctions with $\mathrm{MgO}$ and $\mathrm{Al}_{2} \mathrm{O}_{3}$ tunnel barriers". Physical review letters 99 (22), 226602.

Gerlach, W. and Stern, O. (1922). „Der experimentelle nachweis der richtungsquantelung im magnetfeld". Zeitschrift für Physik A Hadrons and Nuclei 9 (1), 349-352.

Gould, C., Rüster, C., Jungwirth, T., Girgis, E., Schott, G. M, Giraud, R, Brunner, K, Schmidt, G, and Molenkamp, L. W. (2004). „Tunneling anisotropic magnetoresistance: A spin-valve-like tunnel magnetoresistance using a single magnetic layer". Physical Review Letters 93 (11), 117203.

Grünewald, M., Wahler, M., Schumann, F., Michelfeit, M., Gould, C., Schmidt, R., Würthner, F., Schmidt, G., and Molenkamp, L. W. (2011). „Tunneling anisotropic magnetoresistance in organic spin valves". Physical Review B 84 (12), 125208. 
Hall, M. J., Jardine, D. B., Evetts, J. E., Leake, J. A., and Somekh, R. E. (1997). „The role of the magnetic structure and interface scattering in the GMR of magnetic multilayers". Journal of Magnetism and Magnetic Materials 173 (3), 253-258.

Harmon, N. J. and Flatté, M. E. (2014). "Spin relaxation in materials lacking coherent charge transport". Physical Review B 90 (11), 115203 .

Hirsch, J. E. (1999). „Spin Hall effect”. Physical Review Letters 83 (9), 1834-1837.

Ikeda, S., Hayakawa, J., Ashizawa, Y., Lee, Y. M., Miura, K., Hasegawa, H., Tsunoda, M., Matsukura, F., and Ohno, H. (2008). „Tunnel magnetoresistance of $604 \%$ at $300 \mathrm{~K}$ by suppression of Ta diffusion in $\mathrm{CoFeB} / \mathrm{MgO} / \mathrm{CoFeB}$ pseudo-spin-valves annealed at high temperature". Applied Physics Letters 93 (8), 2508.

Johnson, M. and Silsbee, R. H. (1985). „Interfacial charge-spin coupling: Injection and detection of spin magnetization in metals". Physical Review Letters 55 (17), 1790.

Jullière, M. (1975). „Tunneling between ferromagnetic films”. Physics Letters A 54 (3), 225-226.

Karpan, V. M., Giovannetti, G., Khomyakov, P. A., Talanana, M., Starikov, A. A., Zwierzycki, M., van den Brink, J., Brocks, G., and Kelly, P. J. (2007). „Graphite and graphene as perfect spin filters". Physical review letters 99 (17), 176602.

Karpan, V. M., Khomyakov, P. A., Starikov, A. A., Giovannetti, G., Zwierzycki, M., Talanana, M., Brocks, G., van den Brink, J., and Kelly, P. J. (2008). „Theoretical prediction of perfect spin filtering at interfaces between close-packed surfaces of $\mathrm{Ni}$ or $\mathrm{Co}$ and graphite or graphene". Physical Review B 78 (19), 195419.

Kato, Y. K., Myers, R. C., Gossard, A. C., and Awschalom, D. D. (2004). "Observation of the spin hall effect in semiconductors". Science 306 (5703), 1910-1913.

Kawahara, S. L., Lagoute, J., Repain, V., Chacon, C., Girard, Y., Rousset, S., Smogunov, A., and Barreteau, C. (2012). „Large magne- 
toresistance through a single molecule due to a spin-split hybridized orbital". Nano Letters 12 (9), 4558-4563.

Khan, M. N., Henk, J., and Bruno, P. (2008). „Anisotropic magnetoresistance in $\mathrm{Fe} / \mathrm{MgO} / \mathrm{Fe}$ tunnel junctions". Journal of PhysicsCondensed Matter 20 (15), 155208.

Lach, S., Altenhof, A., Tarafder, K., Schmitt, F., Ali, M. E., Vogel, M., Sauther, J., Oppeneer, P. M., and Ziegler, C. (2012). "Metal-organic hybrid interface states of a ferromagnet/organic semiconductor hybrid junction as basis for engineering spin injection in organic spintronics". Advanced Functional Materials 22 (5), 989-997.

Lazić, P., Caciuc, V., Atodiresei, N., Callsen, M., and Blügel, S. (2014). "First-principles insights into the electronic and magnetic structure of hybrid organic-metal interfaces". Journal of Physics-Condensed Matter 26 (26), 263001.

Lazić, P., Sipahi, G. M., Kawakami, R. K., and Žutić, I. (2014). "Graphene spintronics: Spin injection and proximity effects from first principles". Physical Review B 90 (8), 085429.

Lee, Y. M., Hayakawa, J., Ikeda, S., Matsukura, F., and Ohno, H. (2007). „Effect of electrode composition on the tunnel magnetoresistance of pseudo-spin-valve magnetic tunnel junction with a MgO tunnel barrier". Applied Physics Letters 90 (21), 212507.

Levinson, H. J., Greuter, F., and Plummer, E. W. (1983). „Experimental band structure of aluminum". Physical Review B 27 (2), 727.

Liu, A. Y. and Singh, D. J. (1993). „bcc cobalt: Metastable phase or forced structure?" Journal of Applied Physics 73 (10), 6189-6191.

Lüthi, B. (1959). „Longitudinal Magnetoresistance of Metals in High Fields". Physical Review Letters 2 (12), 503.

Malozemoff, A. P. (1986). „Anisotropic magnetoresistance with cubic anisotropy and weak ferromagnetism: A new paradigm". Physical Review B 34 (3), 1853.

Martin, M.-B., Dlubak, B., Weatherup, R. S., Yang, H., Deranlot, C., Bouzehouane, K., Petroff, F., Anane, A., Hofmann, S., 
Robertson, J., Fert, A., and Seneor, P. (2014). „Sub-nanometer atomic layer deposition for spintronics in magnetic tunnel junctions based on graphene spin-filtering membranes". ACS Nano $8(8), 7890-7895$.

Martin, M.-B., Dlubak, B., Weatherup, R. S., Piquemal-Banci, M., Yang, H., Blume, R., Schlögl, R., Collin, S., Petroff, F., Hofmann, S., Robertson, J., Anane, A., Fert, A., and Seneor, P. (2015). „Protecting nickel with graphene spin-filtering membranes: A single layer is enough". Applied Physics Letters 107 (1), 012408.

Martin, Y. and Wickramasinghe, H. K. (1987). „Magnetic imaging by "force microscopy" with $1000 \AA$ A resolution". Applied Physics Letters 50 (20), 1455-1457.

Mathon, J. and Umerski, A. (2001). „Theory of tunneling magnetoresistance of an epitaxial $\mathrm{Fe} / \mathrm{MgO} / \mathrm{Fe}(\mathrm{OO1})$ junction". Physical Review B 63 (22), 220403.

Matos-Abiague, A. and Fabian, J. (2009). „Anisotropic tunneling magnetoresistance and tunneling anisotropic magnetoresistance: Spin-orbit coupling in magnetic tunnel junctions". Physical Review B 79 (15), 155303.

McGuire, T. and Potter, R. L. (1975). „Anisotropic magnetoresistance in ferromagnetic $3 \mathrm{~d}$ alloys". IEEE Transactions on MagnetiCS 11 (4), 1018-1038.

Meservey, R. and Tedrow, P. M. (1971). „Properties of very thin aluminum films". Journal of Applied Physics 42 (1), 51-53.

Moodera, J. S., Kinder, L. R., Wong, T. M., and Meservey, R. (1995). „Large magnetoresistance at room temperature in ferromagnetic thin film tunnel junctions". Physical review letters 74 (16), 3273.

Moorsom, T., Wheeler, M., Khan, T. M., Al Ma'Mari, F., Kinane, C., Langridge, S., Ciudad, D., Bedoya-Pinto, A., Hueso, L., Teobaldi, G., Lazarov, V. K., Gilks, D., Burnell, G., Hickey, B. J., and Cespedes, O. (2014). "Spin-polarized electron transfer in ferromagnet $/ \mathrm{C}_{6} \mathrm{O}$ interfaces". Physical Review B 90 (12), 125311. 
Mott, N. F. (1935). "A discussion of the transition metals on the basis of quantum mechanics". Proceedings of the Physical Society 47 (4), 571.

Mott, N. F. (1936a). „The electrical conductivity of transition metals". 153 (880), 699-717.

Mott, N. F. (1936b). „The resistance and thermoelectric properties of the transition metals". Proceedings of the Royal Society of London. Series A, Mathematical and Physical Sciences 156 (888), 368-382.

Muñoz, R. and Gómez-Aleixandre, C. (2013). „Review of CVD synthesis of graphene". Chemical Vapor Deposition 19, 297-322.

Naber, W. J. M., Faez, S., and van der Wiel, W. G. (2007). „Organic spintronics". Journal of Physics D: Applied Physics 40 (12), R205.

Nguyen, T. A., Lee, J.-U., Yoon, D., and Cheong, H. (2014). „Excitation energy dependent Raman signatures of ABA-and ABCstacked few-layer graphene". Scientific Reports 4, 4630.

Novoselov, K. S., Geim, A. K., Morozov, S. V., Jiang, D., Zhang, Y., Dubonos, S. V., Grigorieva, I. V., and Firsov, A. A. (2004). "Electric field effect in atomically thin carbon films". science 306 (5696), 666-669.

Park, B. G., Wunderlich, J., Williams, D. A., Joo, S. J., Jung, K. Y., Shin, K. H., Olejník, K., Shick, A. B., and Jungwirth, T. (2008). ,Tunneling anisotropic magnetoresistance in multilayer- $(\mathrm{Co} / \mathrm{Pt}) /$ $\mathrm{AlO}_{x} /$ Pt structures". Physical Review Letters 100 (8).

Parkin, S. S. P., Kaiser, C., Panchula, A., Rice, P. M., Hughes, B., Samant, M., and Yang, S.-H. (2004). "Giant tunnelling magnetoresistance at room temperature with $\mathrm{MgO}$ (100) tunnel barriers". Nature Materials 3 (12), 862-867.

Prinz, G. A. (1985). „Stabilization of bcc Co via epitaxial growth on GaAs". Physical Review Letters 54 (10), 1051.

Raman, K. V., Kamerbeek, A. M., Mukherjee, A., Atodiresei, N., Sen, T. K., Lazić, P., Caciuc, V., Michel, R., Stalke, D., Mandal, S. K., Blügel, S., Münzenberg, M., and Moodera, J. S. (2013). 
„Interface-engineered templates for molecular spin memory devices". Nature 493 (7433), 509-513.

Riminucci, A., Prezioso, M., Pernechele, C., Graziosi, P., Bergenti, I., Cecchini, R., Calbucci, M., Solzi, M., and Dediu, V. A. (2013). "Hanle effect missing in a prototypical organic spintronic device". Applied Physics Letters 102 (9), 092407.

Rizzini, A. L., Krull, C., Mugarza, A., Balashov, T., Nistor, C., Piquerel, R., Klyatskaya, S., Ruben, M., Sheverdyaeva, P. M., Moras, P., Carbone, C., Stamm, C., Miedema, P. S., Thakur, P. K., Sessi, V., Soares, M., Yakhou-Harris, F., Cezar, J. C., Stepanow, S., and Gambardella, P. (2014). "Coupling of single, double, and triple-decker metal-phthalocyanine complexes to ferromagnetic and antiferromagnetic substrates". Surface Science 630, 361-374.

Robinson, J. A., LaBella, M., Zhu, M., Hollander, M., Kasarda, R., Hughes, Z., Trumbull, K., Cavalero, R., and Snyder, D. (2011). "Contacting graphene". Applied Physics Letters 98 (5), 053103.

Sáenz, J. J., Garcia, N., Grütter, P., Meyer, E., Heinzelmann, H., Wiesendanger, R., Rosenthaler, L., Hidber, H. R., and Güntherodt, H.-J. (1987). "Observation of magnetic forces by the atomic force microscope". Journal of Applied Physics 62 (10), 42934295 .

Sanvito, S. (2010). "Molecular spintronics: The rise of spinterface science". Nature Physics 6, 562-564.

Schmaus, S., Bagrets, A., Nahas, Y., Yamada, T. K., Bork, A., Bowen, M., Beaurepaire, E., Evers, F., and Wulfhekel, W. (2011). „Giant magnetoresistance through a single molecule". Nature Nanotechnology 6 (3), 185-189.

Simmons, J. G. (1963). „Electric tunnel effect between dissimilar electrodes separated by a thin insulating film". Journal of applied physics 34 (9), 2581-2590.

Slonczewski, J. C. (1989). "Conductance and exchange coupling of two ferromagnets separated by a tunneling barrier". Physical Review B 39 (10), 6995. 
Slonczewski, J. C. (1996). "Current-driven excitation of magnetic multilayers". Journal of Magnetism and Magnetic Materials 159 (12), L1-L7.

Steil, S., Großmann, N., Laux, M., Ruffing, A., Steil, D., Wiesenmayer, M., Mathias, S., Monti, O. L. A., Cinchetti, M., and Aeschlimann, M. (2013). "Spin-dependent trapping of electrons at spinterfaces". Nature Physics 9 (4), 242-247.

Sun, Z., Zhan, Y., Shi, S., and Fahlman, M. (2014). „Energy level alignment and interactive spin polarization at organic/ferromagnetic metal interfaces for organic spintronics". Organic Electronics 15 (9), 1951-1957.

Suzuki, T., Kurahashi, M., Ju, X., and Yamauchi, Y. (2002). „Spin polarization of metal $(\mathrm{Mn}, \mathrm{Fe}, \mathrm{Cu}$, and $\mathrm{Mg}$ ) and metal-free phthalocyanines on an $\mathrm{Fe}(100)$ substrate". Journal of Physical Chemistry B 106 (44), 11553-11556.

Suzuki, T., Kurahashi, M., Ju, X., and Yamauchi, Y. (2004). „Adsorption structure and spin polarization of pentacene on a magnetized $\mathrm{Fe}(100)$ substrate: SPMDS and ERDA study". Surface Science 549 (2), 97-102.

Tang, C. W. and VanSlyke, S. A. (1987). „Organic electroluminescent diodes". Applied Physics Letters 51 (12), 913-915.

Tedrow, P. M. and Meservey, R. (1971). „Spin-dependent tunneling into ferromagnetic nickel". Physical Review Letters 26 (4), 192.

Tedrow, P. M. and Meservey, R. (1973). „Spin polarization of electrons tunneling from films of $\mathrm{Fe}, \mathrm{Co}, \mathrm{Ni}$, and $\mathrm{Gd}^{\prime \prime}$. Physical Review $B 7$ (1), 318.

Thomson, W. (1856). „On the electro-dynamic qualities of metals:Effects of magnetization on the electric conductivity of nickel and of iron". Proceedings of the Royal Society of London 8, 546550 .

Timmermans, F., Liszka, B., Ataç, D., Lenferink, A., Wolferen, H. van, and Otto, C. (2016). „Integrated Raman - FIB - SEM: A 
correlative light and electron microscopy study". Imaging $\mathcal{E} M i$ croscopy (2), 34-37.

Tombros, N., Jozsa, C., Popinciuc, M., Jonkman, H. T., and van Wees, B. J. (2007). „Electronic spin transport and spin precession in single graphene layers at room temperature". Nature 448 (7153), 571-574.

Tran, T. L. A., Çakir, D., Wong, P. K. J., Preobrajenski, A. B., Brocks, G., van der Wiel, W. G., and de Jong, M. P. (2013). „Magnetic properties of bcc-Fe(001)/C-6o interfaces for organic spintronics". ACS Applied Materials \& Interfaces 5 (3), 837-841.

Uhlenbeck, G. E. and Goudsmit, S. (1926). „Spinning electrons and the structure of spectra". Nature 117, 264-265.

Ungár, T. (2004). „Microstructural parameters from X-ray diffraction peak broadening". Scripta Materialia 51 (8), 777-781.

Vogel, J. and Sacchi, M. (1994). „Experimental estimate of absorption length and total electron yield (TEY) probing depth in dysprosium". Journal of Electron Spectroscopy and Related Phenomena 67 (1), $181-188$.

Wang, K., Tran, T. L. A., Brinks, P., Sanderink, J. G. M., Bolhuis, T., van der Wiel, W. G., and de Jong, M. P. (2013). „Tunneling anisotropic magnetoresistance in $\mathrm{Co} / \mathrm{AlO}_{x} / \mathrm{Al}$ tunnel junctions with fcc Co (111) electrodes". Physical Review B 88 (5).

Wang, Y., Zhu, Q., and Zhang, H. (2006). „Fabrication and magnetic properties of hierarchical porous hollow nickel microspheres". Journal of Materials Chemistry 16 (13), 1212-1214.

Watanabe, S., Ando, K., Kang, K., Mooser, S., Vaynzof, Y., Kurebayashi, H., Saitoh, E., and Sirringhaus, H. (2014). „Polaron spin current transport in organic semiconductors". Nature Physics 10 (4), 308-313.

Williams, K. R., Gupta, K., and Wasilik, M. (2003). „Etch rates for micromachining processing-Part II". Journal of microelectromechanical systems 12 (6), 761-778. 
Wong, P. K. J., van Geijn, E., Zhang, W., Starikov, A. A., Tran, T. L. A., Sanderink, J. G. M., Siekman, M. H., Brocks, G., Kelly, P. J., van der Wiel, W. G., and de Jong, M. P. (2013). „Crystalline CoFeB/ graphite interfaces for carbon spintronics fabricated by solid phase epitaxy". Advanced Functional Materials 23 (39), 49334940 .

Xiong, Z. H., Wu, D., Vardeny, Z. V., and Shi, J. (2004). "Giant magnetoresistance in organic spin-valves". Nature 427 (6977), 821824 .

Yabuhara, O., Ohtake, M., Nukaga, Y., and Futamoto, M. (2011). "Preparation and characterization of Co epitaxial thin films on $\mathrm{Al}_{2} \mathrm{O}_{3}$ (OOO1) single-crystal substrates". Journal of Physics: Conference Series 266 (1), 012049.

$\mathrm{Yu}, \mathrm{Z}$. G. (2013). "Suppression of the Hanle effect in organic spintronic devices". Physical Review Letters 111 (1), 016601.

Yuasa, S., Nagahama, T., Fukushima, A., Suzuki, Y., and Ando, K. (2004). "Giant room-temperature magnetoresistance in singlecrystal Fe/MgO/Fe magnetic tunnel junctions". Nature Materials 3 (12), 868-871.

Zhang, Y., Gomez, L., Ishikawa, F. N., Madaria, A., Ryu, K., Wang, C., Badmaev, A., and Zhou, C. (2010). "Comparison of graphene growth on single-crystalline and polycrystalline $\mathrm{Ni}$ by chemical vapor deposition". The Journal of Physical Chemistry Letters 1 (20), 3101-3107.

Zhang, Y., Zhang, L., and Zhou, C. (2013). „Review of chemical vapor deposition of graphene and related applications". Accounts of Chemical Research 46 (10), 2329-2339. 


\section{SAMPLE FABRICATION RECIPE}

The process parameters mentioned in this section are applicable to the equipment available to us in the NanoLab cleanroom facility of the MESA+ Institute for Nanotechnology at the University of Twente. Parameters not specified in the recipe were set to the recommended default values for the used equipment.

SUBSTRATE

Our recipe starts with a silicon wafer that is covered with a $200 \mathrm{~nm}$ to $300 \mathrm{~nm}$ thick nickel layer on top of which graphene is grown using CVD. The wafer is covered with a protective layer of undeveloped photoresist, diced into squares with $11 \mathrm{~mm}$ sides and subsequently cleaned.

Cleaning is typically done by dipping in acetone first, followed by isopropyl alcohol and drying with nitrogen. Depending on the conditions during processing and the waiting time between process steps, additional cleaning steps can be incorporated in the recipe if needed.

BOTTOM ELECTRODES

1. Apply photo resist Spin coat OiR 907/17 positive photoresist at $4000 \mathrm{rpm}$ and bake the resist at $95^{\circ} \mathrm{C}$ for $90 \mathrm{~s}$.

2. UV expose

Align the mask with the sample and expose to UV light for $4.0 \mathrm{~s}$.

3. Post-exposure bake Bake at $120^{\circ} \mathrm{C}$ for $60 \mathrm{~s}$. 
4. Develop resist

\section{Hard bake}

6. Ion-beam etching

\section{Remove resist}

7. $\mathrm{O}_{2}$ Plasma clean
Develop for 29s in OPD-4262, rinse thoroughly with DI water and dry with nitrogen.

Bake at $120^{\circ} \mathrm{C}$ for 120 s to harden resist.

Fully etch away the graphene and nickel layers by argon ion-beam etching with the ion beam aligned perpendicular to the sample surface. In the system available to us this typically takes $55 \mathrm{~min}$ at the default system settings:

$\begin{array}{ll}\text { sample rotation: } & 5 \mathrm{rpm} ; \\ \text { sample angle: } & \mathrm{O}^{\circ} ; \\ \text { neutralizer argon flow: } & 5 \mathrm{sccm} ; \\ \text { neutralizer current: } & 100 \mathrm{~mA} ; \\ \text { plasma RF power: } & 300 \mathrm{~W} ; \\ \text { beam argon flow: } & 5 \mathrm{sccm} ; \\ \text { beam voltage: } & 300 \mathrm{~V} ; \\ \text { beam current: } & 50 \mathrm{~mA} ; \\ \text { accelerator voltage: } & 300 \mathrm{~V} .\end{array}$

Inspect the result of etching under a microscope and if necessary increase etching time to ensure the bottom contacts are well-defined.

Remove damaged resist top layer by oxygen plasma treatment. In our case $30 \mathrm{~s}$ in a 1.25 mbar oxygen atmosphere with a $500 \mathrm{~W}$ plasma. Remove residual resist by regular cleaning in acetone

Remove remaining resist by standard cleaning using acetone and isopropyl alcohol. Very tenacious resist can first be soaked for several minutes in a bath of warm $\left(60^{\circ} \mathrm{C}\right)$ dimethyl sulfoxide. 
INSULATING LAYER

1. Vacuum bake-out Heat samples in situ to $150^{\circ} \mathrm{C}$ to $200{ }^{\circ} \mathrm{C}$ and keep at that temperature for several hours to get rid of gasses and water. Cool to room temperature before proceeding.

2. Deposit $\mathrm{SiO}_{2-\delta}$

Plasma sputter from a silicon dioxide target with no additional oxygen flowing into the system to prevent damage of the graphene. Settings used with the "TCOater" system:

$$
\begin{array}{ll}
\text { argon flow: } & 30 \mathrm{sccm} ; \\
\text { RF plasma power: } & 500 \mathrm{~W} ; \\
\text { deposition time } & 5 \mathrm{~min} .
\end{array}
$$

3. Deposit $\mathrm{SiO}_{2}$

Plasma sputter from a silicon dioxide target with additional oxygen flowing into the system to improve stoichiometry of the deposited layer. Same settings as previous step with the addition of a $5 \mathrm{sccm}$ oxygen flow into the system for a duration of $70 \mathrm{~min}$ to reach a film thickness of $200 \mathrm{~nm}$.

\section{ALIGNMENT MARKERS}

We need these markers, that are visible to both electron and optical microscope, to be able to properly position the EBL designs we make based on optical microscope images.

1. Apply HMDS

2. Photolithography
Spin coat a thin layer of hexamethyldisilazane at $4000 \mathrm{rpm}$ to improve photoresist adhesion.

Identical to first four steps of the bottom electrode fabrication process. That is up 
3. Deposit titanium

5. Lift-off resist to, but not including, the hard bake of the resist.

Plasma sputter a very thin $(2 \mathrm{~nm})$ adhesion layer of titanium. Using the "Sputterke" system with the plasma power set to $100 \mathrm{~W}$ this takes $20 \mathrm{~s}$.

4. Deposit palladium Plasma sputter a $24 \mathrm{~nm}$ palladium layer. Sputtering time in "Sputterke" is 8 o s with the power at $100 \mathrm{~W}$.

Lift-off resist by soaking the sample in warm $\left(60^{\circ} \mathrm{C}\right)$ dimethyl sulfoxide followed by acetone and isopropyl alcohol and drying. Repeat this step until all unwanted metalcovered resist is removed.

CONTACT HOLES

Before the next process steps are carried out, the samples are first investigated under an optical microscope. Images of suitable device locations are recorded that also contain enough alignment markers for proper alignment. EBL designs for the contact holes are created based on these images.

1. Apply EBL resist Spin coat poly(methyl methacrylate) (PMMA) A4 positive photoresist at $4000 \mathrm{rpm}$ and bake the resist at $120^{\circ} \mathrm{C}$ for $90 \mathrm{~s}$.

2. Pattern resist

3. Develop resist
Align the design to the sample and use appropriate settings to expose the resist.

Develop for $3 \mathrm{~s}$ in an 1:3 mixture of methyl isobutyl ketone and isopropyl alcohol, rinse thoroughly with isopropyl alcohol and dry with nitrogen. 
4. BHF wet etching

5. Remove resist
Etch in buffered hydrofluoric acid for $95 \mathrm{~s}$. Thoroughly rinse in DI water and dry.

Remove the EBL resist by soaking in warm $\left(60^{\circ} \mathrm{C}\right)$ dimethyl sulfoxide, followed by acetone and isopropyl alcohol and drying.

TOP ELECTRODES

There are many different possible material choices for the top electrodes depending on the type of measurements to be done. And many different deposition methods can be used.

We deposited the top electrodes on our samples using e-beam evaporation in the "DCA" system. The shape of the top electrodes can be defined by using a shadow mask (piece of metal with openings) placed in front of the sample, as close as possible to the sample surface to avoid shadow effects. But, to be able to make narrower top electrodes in order to limit the area of possible leakage through the insulating oxide layer we used top electrodes defined using EBL for out final devices. The process for creating top electrode using EBL is very similar to that for making the contact holes. The BHF wet etching step is simply replaced by the deposition of the top electrode. When the resist is subsequently removed it lifts off the top electrode material from unwanted locations.

To improve the formation of a closed layer when depositing thin layers we cooled the sample down to $77 \mathrm{~K}$ before deposition of the top electrodes. 

The field of organic spintronics focusses on combining the flexibility and technological advantages of organic electronics with the manipulation and exploitation of the intrinsic spin angular momentum of the electron. Physical phenomena, like magnetoresistance (MR), occurring in (organic) spintronic devices are strongly influenced by, typically spin dependent, interfacial effects such as charge transfer, hybridisation, the formation of interface states, and the broadening and shifting of electronic states. Increasing the understanding of these effects and improving the control over interfacial properties during fabrication are two mutually dependent key topics within the field. The experiments we have performed and that are reported on in this thesis contribute to both these aspects.

Motivated by the prediction of extraordinary phenomena, like perfect spin filtering, we have focussed our attention on the interface between nickel and (multilayer) graphene and, in particular, on the transport perpendicular to this interface. We have explored two fabrication methods for creating devices that contain clean, epitaxial nickel-graphene interfaces.

Graphene grown on nickel using the method of chemical vapour deposition (CVD) typically consists of a polycrystalline nickel film covered with a patchwork of graphene flakes. The properties such as graphene thickness, surface roughness and orientation, and crystal grain size and orientation therefore strongly vary with position. By locally etching away a thick insulating oxide covering the nickelgraphene stack, our process offers the possibility to accurately select the surface area that will be contacted by a top electrode. Although the yield of working devices is seriously hampered by poor adhesion on the inert graphene surface, several working devices have been produced. 
This method also offers large flexibility in the choice of top electrode composition and therefore in the possible measurements that can be done. Devices with an aluminium top electrode have been used to perform transport measurement at temperatures below 10 K in a strong magnetic field. By rotating the sample in the field, out-of-plane TAMR ratios up to $50 \%$ have been measured. These high ratios are attributed to a combination of a strong Rashba effect in nickel-graphene systems and $k$-vector filtering that favours the transport of electrons with a large in-plane wave vector for which this effects is the strongest.

Additionally, we have investigated an alternative fabrication route to grow epitaxial ferromagnetic layers on top of graphene. Simultaneous deposition of nickel, cobalt and boron leads to the formation of an amorphous layer that can be crystallised by annealing. If this process is carried out carefully the weak but finite interaction from the graphene film can be enough to promote epitaxial crystallisation of the layer with a preferential fcc (111) interface orientation. The resulting films show ferromagnetic behaviour, albeit with a reduced magnetisation when compared to bulk nickel.

During our beam times at the MAX-lab synchrotron facility we investigated the effects of molecular overlayers on a thin cobalt film. By comparing the effects of a strong electron donor, tetrathiafulvalene (TTF), with those of a strong electron acceptor, tetracyanoquinodimethane (TCNQ), we specifically focussed on the influence of charge transfer. Both molecular species led to an increase of the ratio of the orbital magnetic moment to the spin magnetic moment of the film. The coercivity, on the other hand, increased by a factor of $\sim 1.5$ after deposition of the donor molecule and was only slightly decreased by the acceptor. The observation of this complementary behaviour shows the feasibility of controlled tuning of the interfacial properties of the cobalt by deposition of a suitable organic semiconductor.

In conclusion, we have shown novel fabrication methods for the realization of devices containing nickel-graphene interfaces, that offer the prospect of obtaining clean, flat and structurally orderer interfaces with a flexible choice of the number of graphene layers. 
Measurements performed on these devices and on hybrid systems with molecular layers have shows fascinating results that prove the importance of interfaces within the field of organic spintronics. 



\section{SAMENVATTING}

Het onderzoeksveld organische spintronica richt zich op het combineren van de flexibiliteit en de technologische voordelen van organische electronica met de manipulatie en uitbuiting van het intrinsieke spin draaimoment van het elektron. Allerlei natuurkundige verschijnselen, zoals bijvoorbeeld magnetoweerstand (MR), die zich voordoen in (organische) spintronicatoepassingen worden sterk beinvloed door, veelal spinafhankelijke, grensvlakeffecten zoals ladingsoverdracht, het ontstaan van grensvlaktoestanden, en de verbreding en verschuiving van elektronentoestanden. Het uitbreiden van de bestaande kennis over deze effecten and het vergroten van de mate van controle over de eigenschappen van grensvlakken tijdens fabricage zijn twee sterk overlappende deelgebieden van het onderzoeksveld. De experimenten die we hebben uitgevoerd en die worden beschreven in dit proefschrift dragen bij aan beide aspecten.

Gemotiveerd door de voorspelling van bijzondere eigenschappen, waaronder volledige spinfiltering, hebben wij onze aandacht gericht op het grensvlak tussen nikkel en grafeen en dan in het bijzonder op transport in de richting loodrecht op dit grensvlak. We hebben twee fabricagemethodes voor de productie van toepassingen met schone, epitaxiale nikkel-grafeen grensvlakken onderzocht.

Met behulp van chemische dampdepositie (CVD) gegroeid grafeen op nikkel bestaat over het algemeen uit een polykristallijne nikkellaag bedekt met een lappendeken van stukjes grafeen. Eigenschappen zoals grafeendikte, oppervlakteruwheid en -oriëntatie, en de afmetingen van de kristalkorrels variëren dan ook sterk van plek tot plek op het oppervlak. Door slechts zeer lokaal een allesbedekkende, isolerend oxide weg te etsen biedt onze fabricagemethode de mogelijkheid om zeer specifiek dat deel van het oppervlak te kiezen waarmee een topelektrode in contact wordt gebracht. En, hoewel de opbrengst van goede apparaten laag is door aanhech- 
tingsproblemen op het grafeenoppervlak, hebben we enkele werkende apparaten weten te produceren.

Tevens biedt deze fabricagemethode grote vrijheid in de keuze voor de samenstelling van de topelektrode en daarmee in het soort metingen dat kan worden uitgevoerd. Apparaten met een aluminium topelektrode zijn gebruik om transportmetingen uit te voeren bij temperaturen onder de $10 \mathrm{~K}$ en in een sterk magneetveld. Door het apparaat te roteren in het magneetveld zijn er uit-het-vlak TAMR ratios tot $50 \%$ gemeten. Deze hoge ratios schrijven we toe aan een samenspel van het sterke Rashba-effect in nikkel-grafeen systemen en het filteren op basis van $k$-vector dat leidt tot een voorkeur voor het transport van elektronen met een grote golfvectorcomponent evenwijdig aan het grensvlak waarvoor dit effect het sterkst is.

Daarnaast hebben we een alternatieve manier om epitaxiale, ferromagnetische lagen op grafeen te groeien onderzocht. Door gelijktijdige depositie van nikkel, kobalt en boor onstaat een amorfe laag die door verhitting kan worden gekristalliseerd. Wanneer dit process gecontroleerd verloopt kan de zwakke maar eindige invloed van de grafeenlaag leiden tot epitaxiale kristallisatie met de voorkeur voor een fcc (111) oriëntatie van het grensvlak. De zo onstane film vertoont ferromagnetisch gedrag, weliswaar met een lagere magnetisatie dan puur nikkel.

Tijdens onze meettijd op de MAX-lab synchrotron faciliteit hebben we de invloed van moleculaire overlagen op een dunne kobaltlaag onderzocht. Door het effect van een sterke elektronendonor, tetrathiafulvaleen (TTF), te vergelijken met dat van een sterke elektronenacceptor, tetracyanochinodimethaan (TCNQ), hebben we ons specifiek gericht op de invloed van ladingsoverdracht. Beide molekulen hebben als gevolg een vergroting van de verhouding tussen het orbitaal magnetisch dipoolmoment en het spin magnetisch dipoolmoment van de laag. De coërciviteit, daarentegen, neemt toe met een factor $\sim 1.5$ na depositie van het donormolekuul en laat slechts een kleine afname zien na depositie van de acceptor. Dit tegengestelde gedrag toont de haalbaarheid aan van het gecontrolleerd regelen van de eigenschappen van het grensvlak van kobalt door deposite van de juiste organische halfgeleider. 
Concluderend: We hebben nieuwe fabricagemethodes laten zien voor toepassingen met nikkel-grafeen grensvlakken die het mogelijk maken om schone, vlakke en structureel geordende grensvlakken te produceren met een flexibele keuze van het aantal grafeenlagen. Metingen aan apparaten met deze grensvlakken en aan hybride systemen met moleculaire lagen leidden tot fascinerende resultaten die het belang van grensvlakken binnen het onderzoeksveld van de organische spintronica onderstrepen. 

Wong, P. K. J., van Geijn, E., Zhang, W., Starikov, A. A., Tran, T. L. A., Sanderink, J. G. M., Siekman, M. H., Brocks, G., Kelly, P. J., van der Wiel, W. G., and de Jong, M. P. (2013). „Crystalline $\mathrm{CoFeB} /$ graphite interfaces for carbon spintronics fabricated by solid phase epitaxy". Advanced Functional Materials 23 (39), 4933-4940.

van Geijn, E., Wang, K., and Jong, M. P. de (2016). „Electronic and magnetic properties of TTF and TCNQ covered Co thin films". Journal of Chemical Physics 144 (17). 

